\title{
The magnetite mineralizations of the Desná Group in the Silesicum, Czech Republic: petrographic, mineralogical, and geochemical studies and their genetic implications
}

\author{
Arno MÜCKE1*, Zdeněk LOSOS² \\ ${ }^{1}$ Experimentelle und Angewandte Mineralogie, Göttinger Zentrum Geowissenschaften, Georg-August-Universität, Goldschmidtstrasse 1, \\ 37077 Göttingen, Germany; arhemucke@gmx.de \\ ${ }^{2}$ Institute of Geological Sciences, Masaryk University, Kotlářská 2, 61137 Brno, Czech Republic; losos@sci.muni.cz \\ * Corresponding author
}

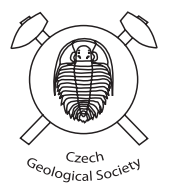

Investigated were occurrences of magnetite-rich rocks (five of which are associated with garnetiferous rocks) situated in the Sobotín Massif (two localities) or in the adjacent gneisses of the Desná Group (six localities). As shown in previous literature, these rocks either belong to iron-formations (six localities) or are of magmatic origin (two localities).

The investigations involved detailed petrographic studies supported by cathodoluminescence microscopy, whole-rock geochemical and mineral (amphibole, biotite, garnet, chlorite, magnetite, ilmenite, carbonate, feldspar and apatite) analyses. The minerals have compositions unknown to iron-formations. Instead they indicate that these may be genetically related to the basic rocks of the ?Devonian Sobotín Massif. Under late-stage and increasing oxygen fugacity conditions of the melt (of the Sobotín Massif), the $\mathrm{Fe}^{3+}$ activity seems to have risen as well. This presumably led to progressive extraction of magnetite from the melt and its enrichment due to gravitational segregation. During this stage, apophyses, mainly composed of amphibole and magnetite, intruded in the gneisses of the Desná Group in the vicinity of the Sobotín Massif. These followed older generations of apophyses that are mainly composed of amphibole only.

The garnet-rich rocks are definitely older than the studied magnetite-rich types and of the same age as the gneisses. The garnetites are inferred to represent original mixtures of precipitates from submarine exhalations and detrital material of continental-derived greywackes. In the absence of local volcanogenic activity, the sedimentary protoliths were composed exclusively of continental-derived material. During a first tectonometamorphic event, the greywacke-derived sediments were transformed into the host-rock gneiss and the mixtures into garnet-rich rocks (= granular iron-formations) identical to coticules.

During a second tectonometamorphic event, all the mentioned rocks were overprinted (alignment and weak foliation of the rocks, chloritization of amphibole and biotite and the formation of actinolite from pre-existing tschermakite and magnesiohornblende, albite, carbonate and epidote). Finally, under retrograde conditions, silicification of varying intensity took place.

Keywords: Desná Group, Sobotín Massif, Silesicum, iron mineralizations, garnetites, petrography, cathodoluminescence microscopy, mineral chemistry, whole-rock geochemistry, genesis

Received: 3 June 2006; accepted 20 December 2007; handling editor: V. Janoušek

\section{Introduction}

\subsection{Geological setting}

The studied iron-mineralizations are hosted by rocks of the Desná Dome, which is a part of the Silesian Domain (NE part of the Bohemian Massif). From a tectonic point of view, Schulmann and Gayer (2000) subdivided the Silesian Domain into four units with the Desná Dome (or the Desná paraautochton) at the base. The Desná Dome is bound by the Culm Foreland Basin in the East and the Keprník nappe in the West, the latter grading via the Červenohorské Sedlo Belt into the rocks of the Desná Dome. Cháb et al. (1984) found evidences of a nappe structure in the Desná Dome, which they subdivided into three sub-units: (1) the autochthonous (or pseudoau- tochthonous) core of the Desná Dome forming the basement built predominantly by gneisses and amphibolites, (2) overlying low-grade metamorphosed metaarkose and metasilstone unit [units (1) and (2) being identical to the Desná paraautochton of Schulmann and Gayer (2000)] and (3) the upper allochthonous units consisting of metagranitoids and overlying quartzites with biotite-muscovite schists of the Devonian Vrbno Group.

\subsection{Iron mineralizations}

Apart from iron ores of the Lahn-Dill type (e.g., Malý Dĕd Mt. belonging to the Vrbno Group), magnetite-rich rocks occur at 12 localities within the biotite-chlorite gneisses of the Desná Dome, geographically identical 


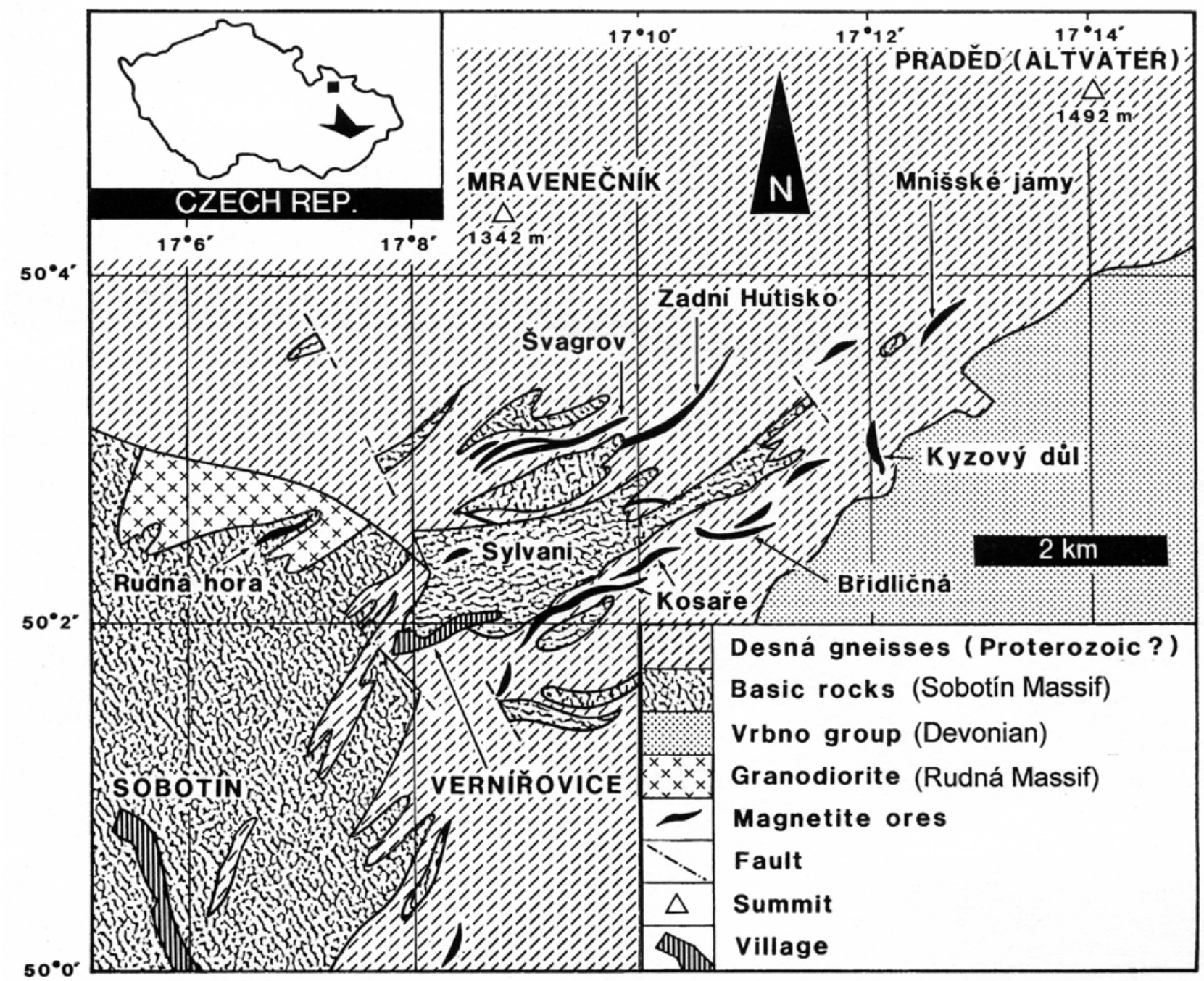

Fig. 1 Geological map of the Sobotín Massif in the gneisses of the Desná Group shown are also the localities investigated in this study (modified from Pouba 1970). The thickness of the ore bodies is slightly exaggerated.

with the High Jeseník Mountains (= Altvatergebirge), Czech Republic. The magnetite-rich rocks occur within, or close to, the basic Sobotín Massif, in an area of about $32 \mathrm{~km}^{2}$, which is slightly stretched in the NE-direction. Eight localities were investigated: Sylvani and Rudná hora within the Sobotín Massif; Kosaře, Břidličná and Kyzový důl in the southern part of the area; Švagrov and Zadní Hutisko in the northern part and Mnišské jámy at the north-eastern extremity of the mineralized region (Fig. 1).

The ores were mined between the $17^{\text {th }}$ and $19^{\text {th }}$ centuries, up to 1865 . Kretschmer (1911) and Sellner (1930) published the first reports. In these publications the ore mineralization at Sylvani was inferred to be of magmatic segregation origin (Kretschmer 1911). On the other hand, that of Mnišské jámy was assigned by Sellner (1930) to the so-called Bändererz (= banded ore) formed by con- tact metasomatism, a view which was later confirmed by Berderke (1938). In the years 1952-1960 geological and geophysical research led to the re-discovery of additional old deposits noted above. All these were intensively investigated by Pouba during the period 1952 to 1970 , when electron microprobe was not yet available. Therefore, investigations of Pouba (1970) included detailed petrography, whole-rock chemistry, photomicrographs, sketches and many diagrams, which contributed substantially to the knowledge of the deposits.

Pouba (1970) distinguished the following ore types: (1) quartz-magnetite ore, which may be associated with chlorite (biotite) or amphibole; (2) Mn-rich garnet-magnetite ore; (3) amphibole-magnetite ore including banded and massive types resulting from mobilization (mobilized ore) or recrystallization (regenerated ore); (4) carbonate-magnetite ore; and (5) sulphide ore. 
The ore was considered by Pouba (1970) with Pouba and Zoubek (1985) to belong to iron-formations and to be of the Sydvaranger type (Pouba 1960), named after the Norwegian deposit of Early Karelian age (2.5 to $2.6 \mathrm{Ga}$ ), which is of submarine-sedimentary exhalative origin. The term "Sydvaranger type", unknown in the international nomenclature, is identical to the Algoma type of banded iron-formations (Gross 1980). The sedimentary protoliths underwent two metamorphic processes (Pouba 1970):

1. Caledonian or Late Precambrian tectonometamorphic event, during which the structure of the sediments was widely preserved, but primary Fe-silicates, hydrohematite or hematite were converted into magnetite, quartz and silicates.

2. Retrograde greenschist-facies Variscan tectonometamorphic event and younger magmatic processes, in course of which was magnetite partially mobilized leading to the formation of compact accumulations of this mineral.

Pouba (1970) stated that the origin of amphibole of the banded magnetite-quartz and magnetite-amphibole rocks is unclear. The occurrence of amphibole may either reflect the original composition of the protolith or may be due to the influence of basic intrusions of the Sobotín Massif (Pouba 1970). The massif remains undated but is inferred by many workers to have been emplaced in Devonian times (395 to 345 Ma ago) (Pouba 1960; Fiala et al. 1980; Cháb et al. 1984). These intrusions, rich in amphibole and magnetite, penetrated the gneiss complex of the Desná Group in the form of apophyses, sills or dykes and are parallel to the ore layers. Only in cases when they were cross-cutting the ore bands, Pouba (1970) noted that the amphibole-magnetite-bearing apophyses were younger. This was the reason why Pouba (1960) had no doubt that an intrusive origin of the magnetite-rich deposits within the gneisses can be completely ruled out. On the other hand, Pouba (1970) followed the opinion of Kretschmer (1911), who believed that the deposits of Sylvani and Rudná hora are connected with basic intrusions of the Sobotín Massif.

In brief publications, Mücke and Losos (1999, 2000 and 2002) presented an opinion that the ores are not identical to iron-formations but may be related to intrusions of the Sobotín Massif. The ores have been interpreted to be of segregation origin and emplaced due to filter pressing in the form of apophyses that penetrated the gneisses of the Desná Group. The garnet-rich rocks seem to be older than the magnetite mineralizations and belong most probably to the Algoma type iron-formation.

Fojt $(2002,2003)$ and Fojt et al. $(1997,2004)$ followed Pouba $(1960,1970)$ in explaining the magnetite ores of the Desná Group as meta-exhalites genetically related to the younger Lahn-Dill type deposits of the same area. Due to observations of the effect of thermal metamor- phism caused by apophyses related to the Sobotín Massif, Fojt et al. (2004) believed that the iron-mineralizations are older (of Proterozoic age) than the intrusion of the ?Devonian Sobotín Massif. The manganese-rich garnetiferous rocks, considered by Pouba (1970) with Zimák and Kropác (2006) to be of the same age as the iron mineralizations, were postulated by Fojt (2003) to be younger as they were observed solely in the hanging wall layers of the deposits, similar to the sulphide enrichments. However, their genesis and age were not discussed.

Our paper presents data indicating that the magnetiterich rocks of the Desná Group could have emplaced as apophyses originating from the Sobotín Massif and that the garnetites, which are older, are most likely of submarine-volcanogenic origin.

\section{Sampling and sample description}

\subsection{Origin of samples}

In total c. 50 samples were collected during several field trips in the years 1992-1998 from 6 deposits (Kosaře, Švagrov, Břidličná, Zadní Hutisko, Kyzový důl and Mnišské jámy). The samples of Sylvani and Rudná hora are from the collection of the Moravian Museum, Brno, whereas some others were kindly donated by B. Fojt, Brno and Z. Pouba, Praha. At present, the former six deposits are not opened and therefore the samples were collected sporadically within the area of the former mines or in the stockpiled materials (e.g., Švagrov). Given this situation, we could not obtain data on the distribution of the various rock types, shapes of their bodies and their thicknesses. The present study concentrated on obtaining detailed petrographic and mineralogical data, established from more than 60 polished and about 50 covered thin sections.

\subsection{Sample description}

Apart from magnetite, the main minerals of the rocks, macroscopically mostly not identifiable, were determined by microscopy and are summarised below.

\subsubsection{Rocks of the Sobotín Massif}

In Sylvani and Rudná hora, three rock types were observed: (1) hornblendite (Rudná hora) and hornblendite grading abruptly into (2) rocks which are composed mainly of magnetite (about 50 vol. \%) and amphibole (Sylvani; Fig. 2), and (3) rocks consisting of alternating bands of magnetite + amphibole and quartz (Sylvani, Fig. 3) sometimes slightly folded (Rudná hora, Fig. 4). 


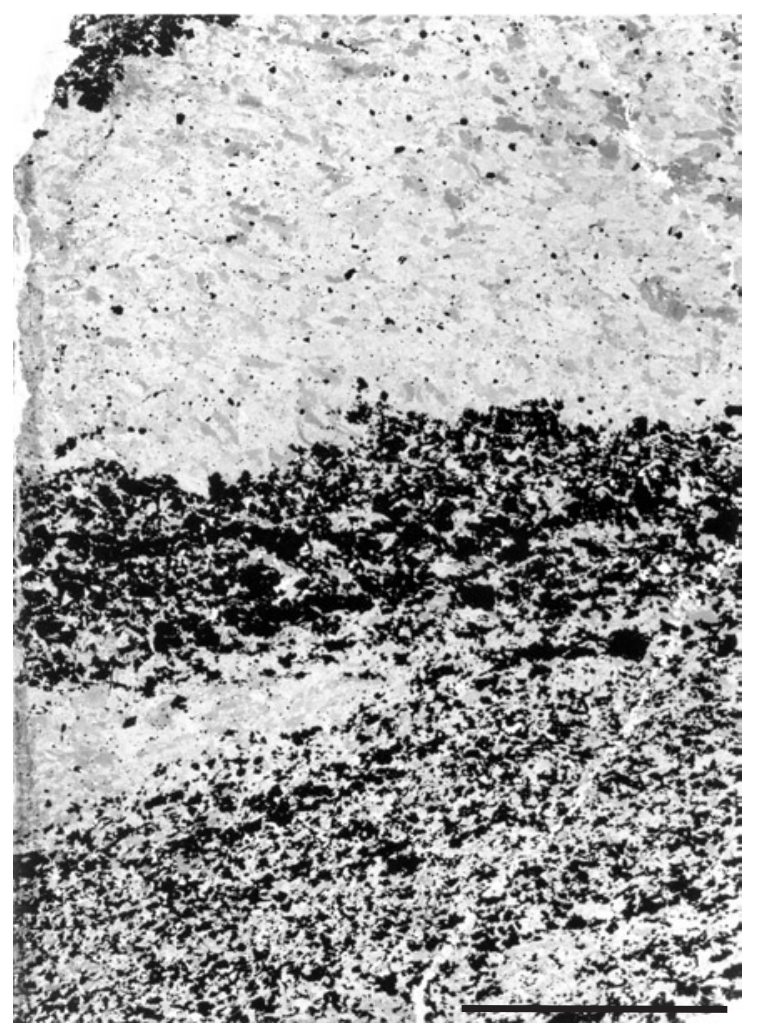

Fig. 2 Rock consisting of magnetite and amphibole (lower part) in contact to metahornblendite. Transmitted light, scale bar $1 \mathrm{~cm}$, Sylvani.

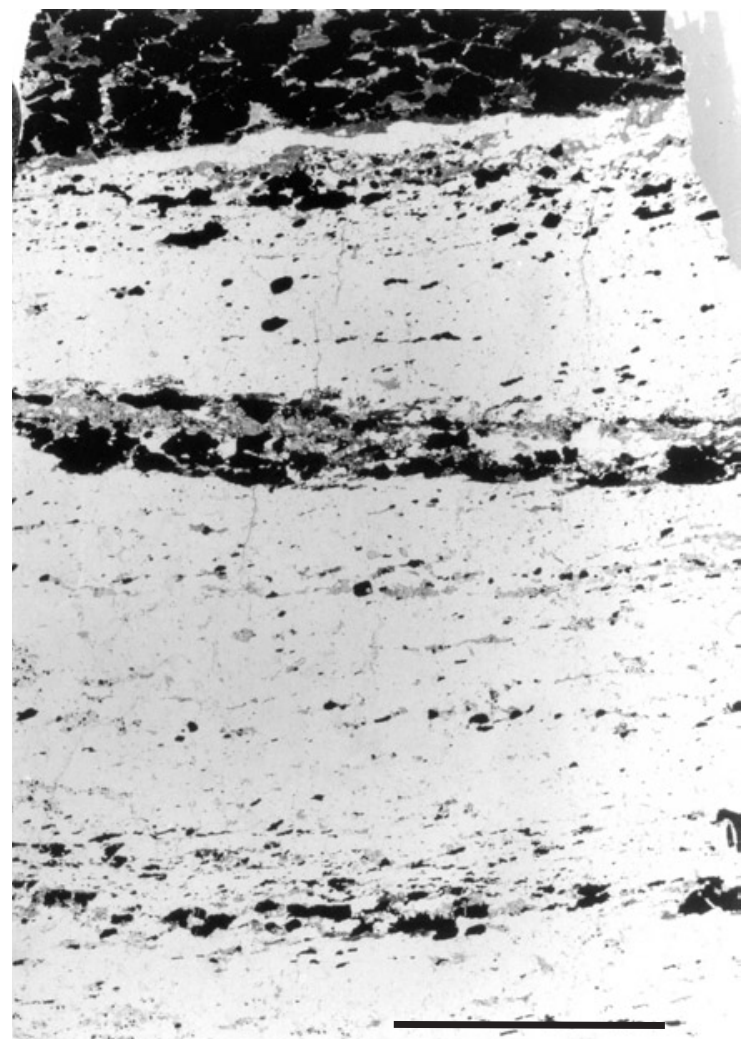

Fig. 3 Alternating bands consisting of magnetite + amphibole and quartz. Note the presence of corroded grains of amphibole and magnetite (black) in quartz. Transmitted light, scale bar $1 \mathrm{~cm}$, Sylvani.

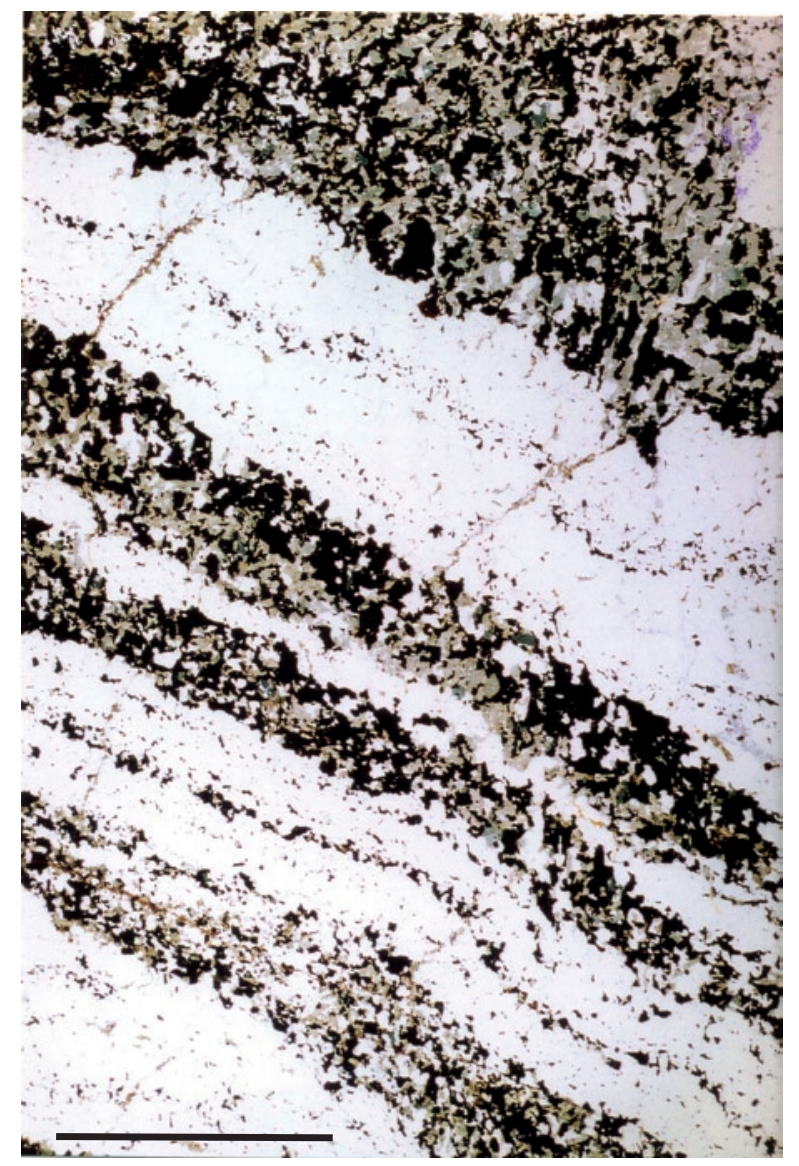

Fig. 4 Alternating, slightly folded bands of magnetite + amphibole and quartz. Transmitted light, scale bar $1 \mathrm{~cm}$, Rudná hora.

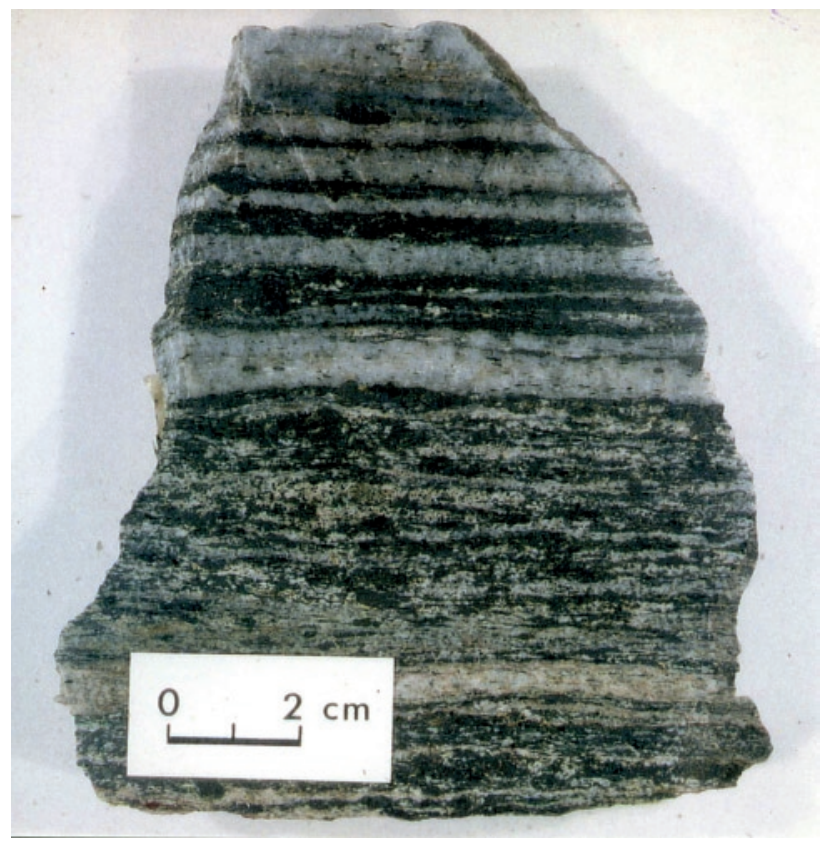

Fig. 5 Banding formed by magnetite + amphibole and quartz. Note that the thickest band (lower part) consists of lensoidal, parallel arranged bodies of magnetite + amphibole that are embedded in a quartz groundmass (see Fig. 6). Kosaře. 


\subsubsection{Rocks in the gneisses of the Desná Group}

The rocks from Kosaře consist of alternating, light and dark bands (Fig. 5). The light bands (thickness mainly in the $\mathrm{mm}$ range, rarely up to $1-2 \mathrm{~cm}$ ) are made of quartz and the dark of magnetite + amphibole (the thickest are c. $10 \mathrm{~cm}$ wide). Especially those bands with high thickness are composed of small, parallel aligned, lensoidal bodies (30 to 80 vol. \%), which are embedded in quartz. The lensoidal bodies are built by magnetite and minor quantities of amphibole (Fig. 6). One sample, consisting of slightly folded magnetite-amphibole bands alternating with quartz bands, contains several (five), slightly broken bands (up to $2 \mathrm{~mm}$ thick) of garnetite.

The rocks at Švagrov, mainly magnetite-rich, occur mostly in the form of alternating, slightly folded, dark and light bands (thickness in the mm-range). The light bands consist of quartz, whereas the dark ones (thickness in the range between $\mathrm{mm}$ and first $\mathrm{cm}$ ) are composed of magnetite (up to 80 vol. \%) and amphibole. Another magnetite-rich rock type consists of irregular magnetite-amphibole bands (several $\mathrm{cm}$ thick), which show schlieren-like patterns, and inclusions of quartz lenses. These bands may alternate with quartz and may either

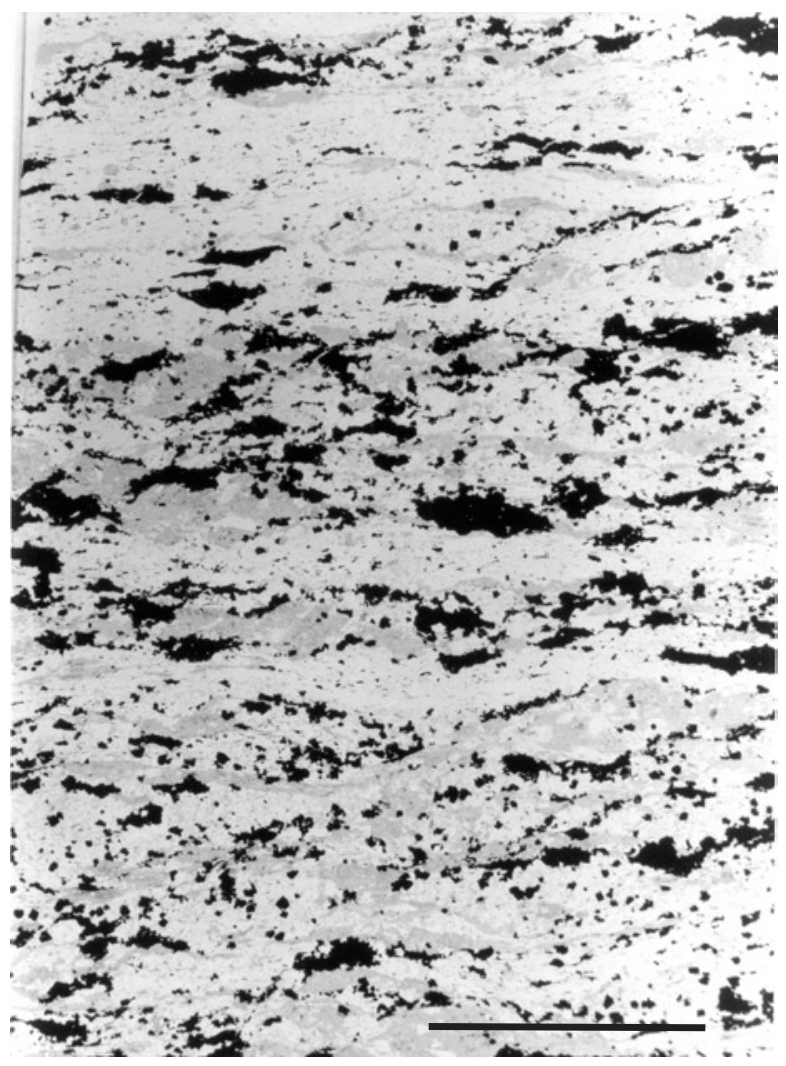

Fig. 6 Aligned and parallel arranged lensoidal aggregates of magnetite (black) and amphibole (light grey) in quartz (white). Transmitted light, scale bar $1 \mathrm{~cm}$, Kosaře. grade into gneiss or garnetite. Metahornblendite is also an abundant rock type, which is often associated with quartz (up to 60 vol. \%) containing irregularly shaped, slightly elongated amphibole aggregates.

In the deposits of Břidličná, Kyzový důl and Mnišské jámy occur magnetite and subordinately chlorite with biotite in dark bands. They are, likewise at Sylvani and Rudná hora (see Figs 3 and 4), slightly (Fig. 7) or strongly folded and deformed. These bands, parallel to each other, are included in quartz. The magnetite-rich bands have thicknesses ranging from $1 \mathrm{~mm}$ to $3 \mathrm{~cm}$ (Fig. 8). In some bands, especially the thick ones, magnetite seems to be broken and aligned (Fig. 9). The proportion of the magnetite-rich bands to quartz varies between $1: 10$ and 1:3. Garnetite occurs in Mnišské jámy in the form of irregularly distributed, schlieren-like "bands" within quartz or in contact to the gneiss host. Magnetite-chlorite(biotite) rocks were not observed in the garnetite-bearing sample.

Nearly all samples from Zadní Hutisko contain garnetite. They belong to two types. (1) Rocks consisting of alternating bands, which are unfolded and strictly parallel to each other. This rock type occurs in two varieties: (1a; rarely observed) dark bands (composed of magnetite + amphibole; thickness varying from $0.5 \mathrm{~mm}$ to

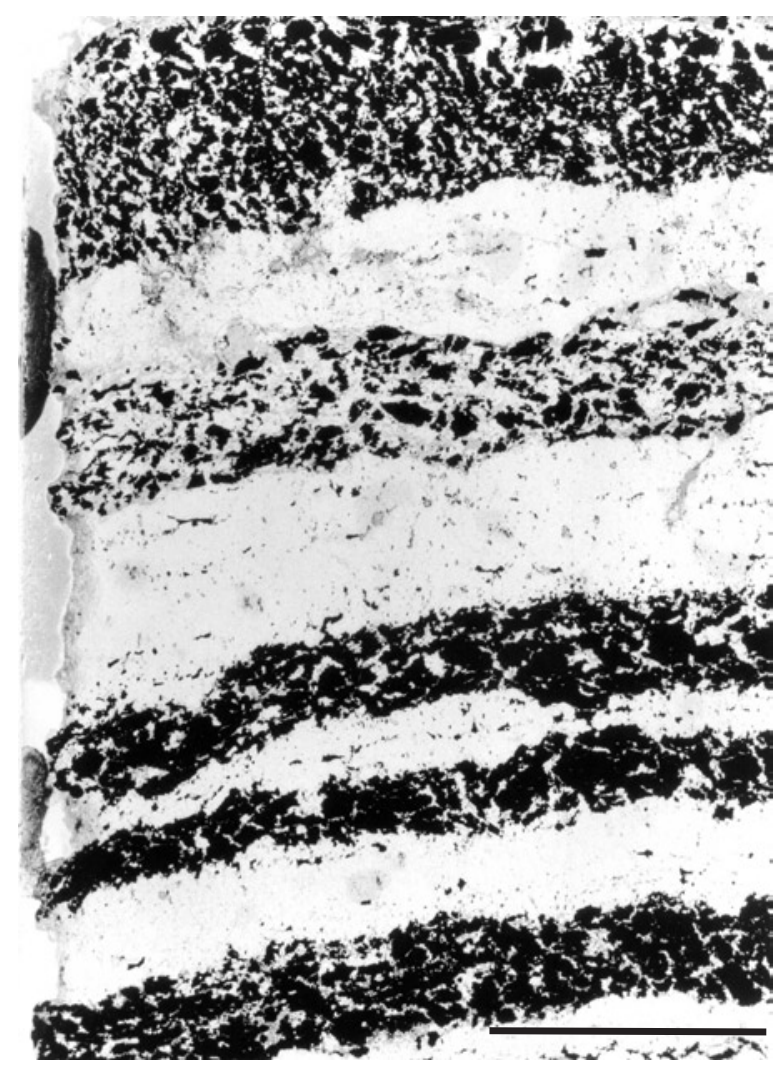

Fig. 7 Slightly folded bands of magnetite-chlorite(-biotite) separated by bands consisting of quartz. Transmitted light, scale bar $1 \mathrm{~cm}$, Mnišské jámy. 


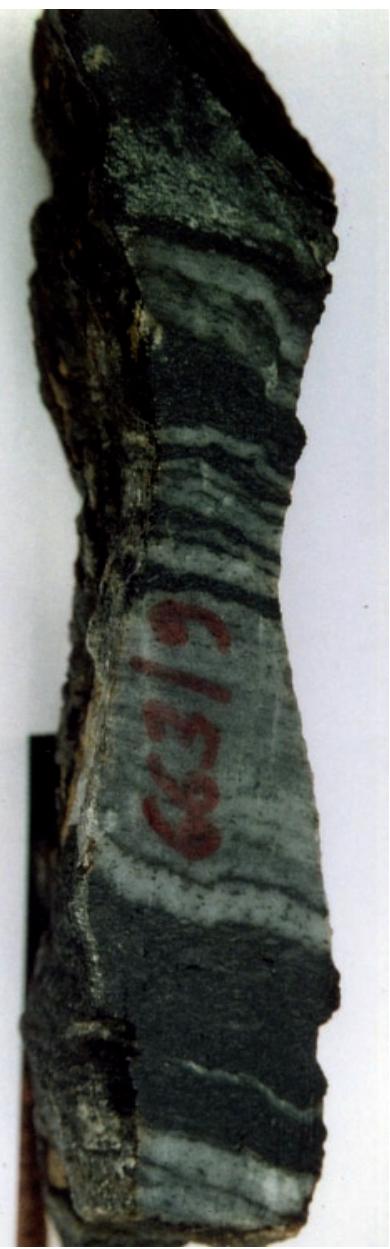

Fig. 8 Slightly folded bands consisting predominantly of magnetite (with minor chlorite and biotite) alternating with quartz. Length of the sample $20 \mathrm{~cm}$. Kyzový důl.

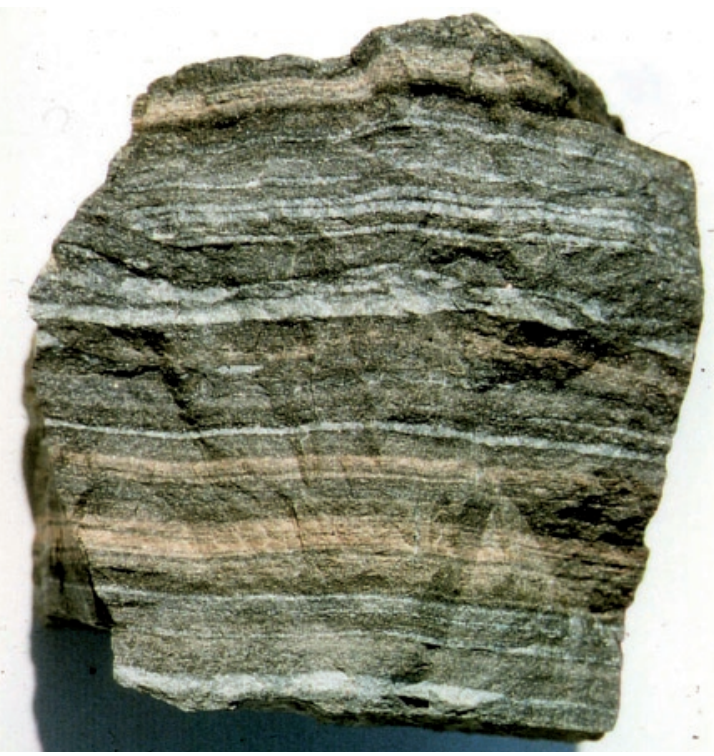

Fig. 10 Alternating bands of pink to browish garnetite and dark magnetite + biotite + chlorite. The dark bands are separated by thin quartz bands. Size of the sample $11 \times 9 \mathrm{~cm}$, Zadní Hutisko.

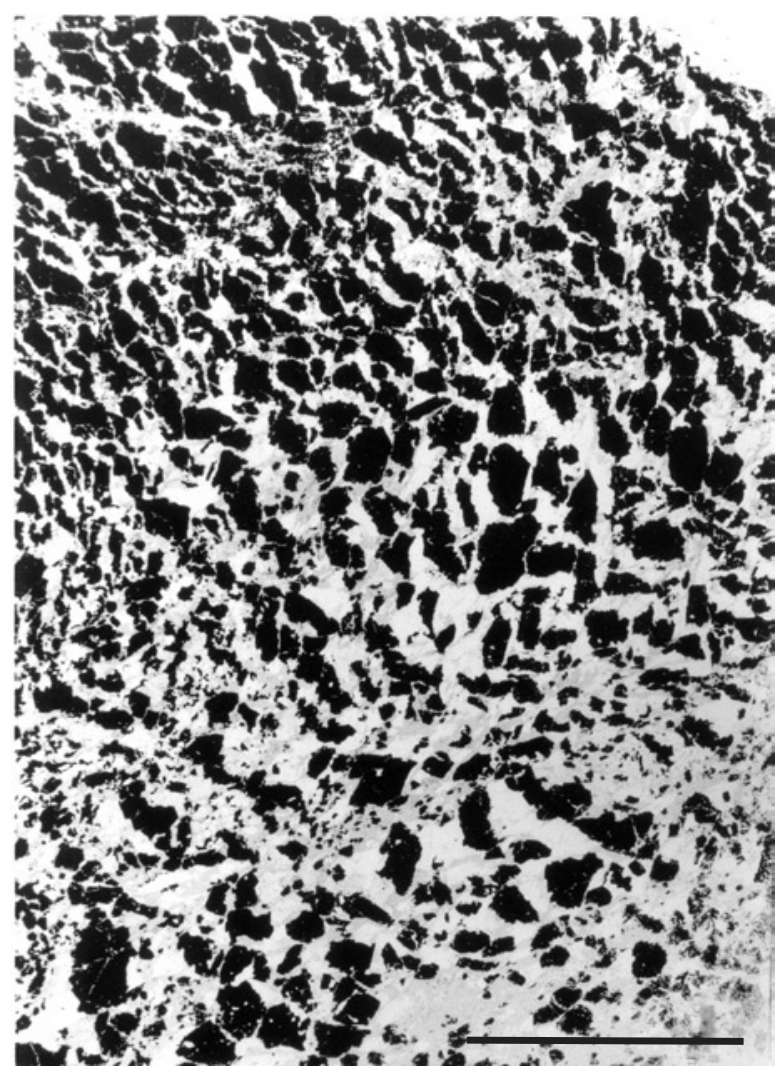

Fig. 9 Broken and aligned magnetite grains of a thick band (detail of the thickest band on Fig. 8) embedded in chlorite and biotite. Transmitted light, scale bar $1 \mathrm{~cm}$, Kyzový důl.

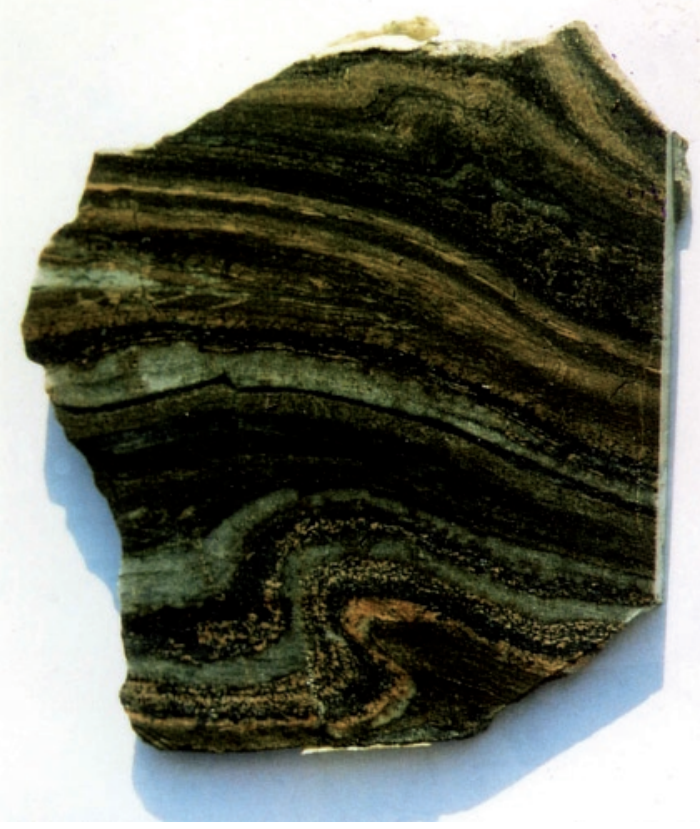

Fig. 11 Alternating, folded bands consisting of garnetite (dark-brown to brown), magnetite-rich garnetite, magnetite-chlorite-biotite (dark) and quartz (light). Size of the sample $10.5 \times 9 \mathrm{~cm}$, Zadní Hutisko. 
$2 \mathrm{~mm}$ ) separated by quartz bands (thickness up to $2 \mathrm{~cm}$ ); (1b) pink to brownish bands of garnetite (up to $2 \mathrm{~cm}$ thick) sandwiched in between dark bands (composed of magnetite + chlorite + biotite, thickness up to $3 \mathrm{~cm}$ ). The latter are intersected by quartz bands of varying thickness $(0.5$ to $3 \mathrm{~mm}$ ) (Fig. 10). (2) Rocks with alternating and folded bands. These bands consist predominantly of garnetite (up to $2.5 \mathrm{~cm}$ ) or garnetite with relatively high proportion of magnetite grains, rarely magnetite-chlorite-biotite $(1$ to $2 \mathrm{~cm})$, quartz $(0.5 \mathrm{~cm})$ and locally also gneiss (Fig. 11).

Outcropping rocks consisting of coarse-grained amphibole and varying quantities of fine-grained feldspar (up to 60 vol. \%) may also occur at Zadní Hutisko in the form of veins cutting the gneiss. Clearly discernible is that feldspar replaces amphibole.

\section{Methods}

\subsection{Whole-rock analyses}

In total 41 whole-rock analyses were determined by PHILLIPS PW 1480 XRF at Georg-August-University of Göttingen. International standards were used for calibration (published in: Geostandard Newsletters, Vol. XIII, Special Issue, 1989). The XRF analyses were augmented by the determination of the $\mathrm{H}_{2} \mathrm{O}^{-}$(heating at $110^{\circ} \mathrm{C}$ ) and $\mathrm{H}_{2} \mathrm{O}^{+}$or the LOI contents (heating at $1000^{\circ} \mathrm{C}$ ). The $\mathrm{Fe}^{2+}$ was determined by titration in sulphuric acid in the presence of $\mathrm{KMnO}_{4}$ (Heinrichs and Herrmann 1990). Eight whole-rock samples were selected for REE analyses carried out at Georg-August-University of Göttingen by a Perkin-Elmer SCIEX (Concord, Ontario, Canada) ELAN DRC II ICP-MS spectrometer. Dissolution of rock powders was carried out by stepwise acid attack with $\mathrm{HF}, \mathrm{HClO}_{4}$, and $\mathrm{HNO}_{3}$ at $180^{\circ} \mathrm{C}$ for about 12 hours. All samples are normalized using internal standardization by Ge, Rh, In, and Re. Reproducibility was better than 1-2\%, accuracy was checked by comparison with international Standard JA-2 [GSJ Geochemical Reference samples: National Institute of Advanced Industrial Science and Technology (AIST)] and did not exceed 10-15\% deviation. All REE were corrected for Ba, REE plus oxygen interferences especially on ${ }^{151} \mathrm{Eu}$ by ${ }^{135} \mathrm{Ba}^{16} \mathrm{O}$ and ${ }^{157} \mathrm{Gd}$ by ${ }^{141} \operatorname{Pr}^{16} \mathrm{O}$.

\subsection{Cathodoluminescence microscopy}

Cathodoluminescence (CL) studies have been carried out with a high-power HC3-LM Simon-Neuser CL microscope (Neuser et al. 1995). The operating accelerating voltage was $14 \mathrm{kV}$ and the beam current less than $1 \mathrm{~mA}$, corresponding to $20-40 \mu \mathrm{A} / \mathrm{mm}^{2}$ beam current density.

\subsection{Mineral chemistry}

Electron microprobe analyses were carried out at the Institut für Mineralogie und Mineralische Rohstoffe of the University of Clausthal-Zellerfeld. The analytical data are summarized in the upper part of the Tables 2-10 and 15 . Wavelength dispersive microprobe analyses were performed using a CAMECA SX 100 instrument (correction program PHIRHO-Z), equipped with five WDS detectors. For quantitative measurements, $15 \mathrm{kV}$ acceleration voltage, $20 \mathrm{nA}$ beam current on the Faraday cup and $5 \mu \mathrm{m}$ defocused beam were chosen. Counting times ranged between 12 and $22 \mathrm{~s}$ on the peak and total background analyzing the X-ray $\mathrm{K}_{\alpha}$ signals of $\mathrm{Na}, \mathrm{Mg}$, $\mathrm{Al}, \mathrm{Si}, \mathrm{K}, \mathrm{Ca}, \mathrm{Ti}, \mathrm{Mn}$ and $\mathrm{Fe}$. The PAP matrix correction method for the raw counts was employed (Pouchou and Pichoir 1984). The following standards were used for the analyses: kaersutite ( $\mathrm{Na}, \mathrm{Mg}, \mathrm{Al}, \mathrm{Si}, \mathrm{K}, \mathrm{Ca}), \mathrm{TiO}_{2}$ (Ti), almandine $(\mathrm{Mn})$ and hematite $(\mathrm{Fe})$.

The amphibole analyses (Tabs 2 and 3) were recalculated as proposed by Schumacher (1997) and classified following Leake et al. (1997). According to Schumacher's calculation scheme, the $\mathrm{Fe}^{3+}$ values were averaged and calculated on the basis of electron neutrality for the formulae. The at. $\%$ of $\mathrm{Fe}^{2+}$ and $\mathrm{Fe}^{3+}$ were transformed into wt. \% (expressed as $\mathrm{Fe}_{2} \mathrm{O}_{3 \text { calc }}$ and $\mathrm{FeO}_{\text {calc }}$ ) and added to the analytical sum instead of $\mathrm{FeO}_{\text {analyzed }}$. Deduced from the formula of amphibole, equivalent proportions of water were calculated, transformed into wt. \%, and added to the analytical sum.

The biotite formulae (Tab. 4) were calculated on the basis of 8 cations. The calculation of electron neutrality for the formula and the determination of the water content are identical to the calculation procedure of amphibole. The presentation of the analytical data follows the proposal of Rieder et al. (1998). Based on these calculations, equivalent proportions of water were deduced, transformed into wt. \%, and added to the analytical sum.

Chlorite analyses (Tab. 5) were calculated on the basis of 10 cations including minor $\mathrm{CaO}, \mathrm{Na}_{2} \mathrm{O}$ and $\mathrm{K}_{2} \mathrm{O}$. Two cases occur in the procedure of electron neutrality calculation for the chlorite formula $\left(\mathrm{Fe}^{2+}, \mathrm{Mg}, \mathrm{Fe}^{3+}, \mathrm{Al}\right)_{6}[\mathrm{OH})_{8} /$ $\left.(\mathrm{Si}, \mathrm{Al})_{4} \mathrm{O}_{10}\right]$ :

1. The valences of the anions $(=28)$ are higher than the sum of the cations (assuming that iron is exclusively $\left.\mathrm{Fe}^{2+}\right)$. The excess of negative charge is compensated by introduction of a certain amount of $\mathrm{Fe}^{3+}$.

2. The valency of the anions $(=28)$ is lower than the total of the cations (on the assumption that iron is exclusively $\mathrm{Fe}^{2+}$ ). The deficiency of negative charges is compensated by the introduction of $\mathrm{O}^{2-}$ instead of $\mathrm{OH}^{-}$. As a consequence, $\mathrm{Fe}^{3+}$ does not occur.

Garnet formulae (Tab. 6) were calculated on the basis of 8 cations according to the formula $\left(\mathrm{Fe}^{2+}, \mathrm{Mn}^{2+}, \mathrm{Ca}\right)_{3}$ 
$\left(\mathrm{Al}, \mathrm{Fe}^{3+}\right)_{2}\left(\mathrm{SiO}_{4}\right)_{3}$. The proportions of $\mathrm{Fe}^{3+}$ were estimated from the amount of $\mathrm{Al}^{\mathrm{VI}}$ and its difference from 2 .

Magnetite analyses (Tab. 7) were calculated on the basis of 3 cations, assuming the general spinel formula $\left(\mathrm{Fe}^{2+}, \mathrm{Mn}^{2+}\right)_{1.00}\left(\mathrm{Fe}^{3+}, \mathrm{Cr}, \mathrm{Al}, \mathrm{Ti}\right)_{2.00} \mathrm{O}_{4}$. The proportions of $\mathrm{Fe}^{2+}$ and $\mathrm{Fe}^{3+}$ were obtained from end-member mol. \%.

Ilmenite formulae ( $\mathrm{Fe}, \mathrm{Mn}, \mathrm{Mg}, \mathrm{Ca}) \mathrm{TiO}_{3}$ (Tab. 8) were calculated on the basis of 2 cations. The results are presented as mol. \% of the end-members - pyrophanite, geikielite, "perovskite", hematite and eskolaite.

\section{Petrography}

\subsection{Rocks of the Sobotín Massif}

\subsubsection{Sylvani and Rudná hora}

In Sylvani the ore-rich rocks consist of magnetite and amphibole (magnetite-amphibole rocks; Fig. 12a). The two minerals occur mainly in roughly equal proportions. The magnetite crystals, often occurring as aggregates, are coarse-grained ( 0.05 to $0.65 \mathrm{~mm}$, on average $0.3 \mathrm{~mm}$ ), not martitized, subhedral to euhedral and have smooth but irregular to embayed grain boundaries. The amphibole grains may be slightly aligned and have length varying between 3.0 and $0.2 \mathrm{~mm}$ (width 0.6 to $0.05 \mathrm{~mm}$ ). The magnetite-amphibole rocks in places pass into nearly monomineralic hornblendites (Sylvani; Fig. 12b). Another, rare rock type consists of bands of magnetite + amphibole and quartz, sometimes slightly folded. The contacts between alternating bands are discontinuous and characterized by corroded amphibole (Fig. 12c) and, better preserved, magnetite crystals. The quartz bands contain magnetite and subordinate amphibole relics.

At Sylvani, magnetite may contain spinel lamellae oriented parallel to (100) (up to $25 \mu \mathrm{m}$ long) and rare lamellae of ilmenite oriented parallel to (111) (up to 100 $\mu \mathrm{m}$ long). However, ilmenite mainly occurs along magnetite grain boundaries in the form of elongated (up to $0.15 \mathrm{~mm}$ long) to stubby crystals along magnetite grain boundaries or in interstitial aggregates. Rarely, these ilmenite crystals contain fine, nearly sub-microscopic exsolutions of hematite and corundum and may be replaced by titanite. Ilmenite is relatively abundant at Sylvani but scarce at Rudná hora. Other minerals are biotite (only in Sylvani), chlorite (only in Rudná hora), apatite, epidote, and carbonate.

One of the samples from Rudná hora corresponds to hornblendite containing minor phases of feldspar, epidote, apatite and rare magnetite.

\subsection{Rocks in the gneisses of the Desná Group}

\subsubsection{Kosaře}

The rocks at Kosaře contain amphibole and magnetite. One facies that is rarely developed consists of alternating bands composed of magnetite + amphibole and quartz. However, in the predominant facies magnetite and amphibole form small parallel, lenticular, aggregates, which are embedded in quartz. The latter two minerals occur in large grains (amphibole up to $1.6 \mathrm{~mm}$ by $0.7 \mathrm{~mm}$; magnetite 0.03 to $0.3 \mathrm{~mm}$ ). Amphibole is in places replaced by epidote (Fig. 12d) and has corroded grain boundaries, while magnetite is affected only weakly. Other minerals are biotite, chlorite, feldspar (albite and K-feldspar), ilmenite, hematite (all in grains $c$. 5 to $10 \mu \mathrm{m}$ across) and apatite. Apatite in rounded grains is accessoric and only locally enriched (Fig. 12e). Ilmenite forms relics within newly-formed blitz-textured intergrowth of hematite and rutile.

Garnetite, a rare rock type at Kosaře, consists of fine-grained garnet (about 80 vol. \%; grain size 0.01 to $0.02 \mathrm{~mm}$ ), chlorite and magnetite set in a quartz groundmass. The garnetite is closely associated with the above-mentioned amphibole-magnetite rocks and may also grade into the host gneiss. At the contact between garnetite and magnetite-amphibole rock, the garnet crystals are overgrown by amphibole and magnetite.

\subsection{2. Švagrov (Zdeněk and Václav Mines)}

Most of the rocks are magnetite-amphibole types, which may contain bands of garnet-rich rocks (garnetite) several $\mathrm{mm}$ thick (Fig. 12f). The contact between magnetite-amphibolite bands and garnetite is relatively sharp. However, along a small zone (in the mm-range), the garnet crystals are overgrown by amphibole and magnetite (Figs $12 \mathrm{f}$ and 13a). The garnet crystals are small ( 0.1 to $0.03 \mathrm{~mm}$; on average $0.02 \mathrm{~mm}$ ) and may constitute up to 95 vol. \% of the rock. Other minerals in the garnetite are quartz in the groundmass, chlorite, magnetite $(0.03$ to $0.015 \mathrm{~mm}$ ), ilmenite carrying hematite exsolutions, and $\mathrm{K}$-feldspar. Compared to the garnetites, the magnetite-amphibole rocks are coarser grained (magnetite 0.04 to $0.20 \mathrm{~mm}$; on average $0.08 \mathrm{~mm}$; amphibole 0.2 to $0.6 \mathrm{~mm}$, on average $0.4 \mathrm{~mm}$ ). Their magnetite : amphibole ratio varies between $1: 9$ and $3: 2$ but is mostly $1: 1$. Amphibole may be replaced by chlorite (Fig. 13b). In contact with the quartz bands, amphibole and, to a lesser degree, magnetite, are corroded and replaced by quartz (Fig. 13a). Within the quartz bands, relics of magnetite 

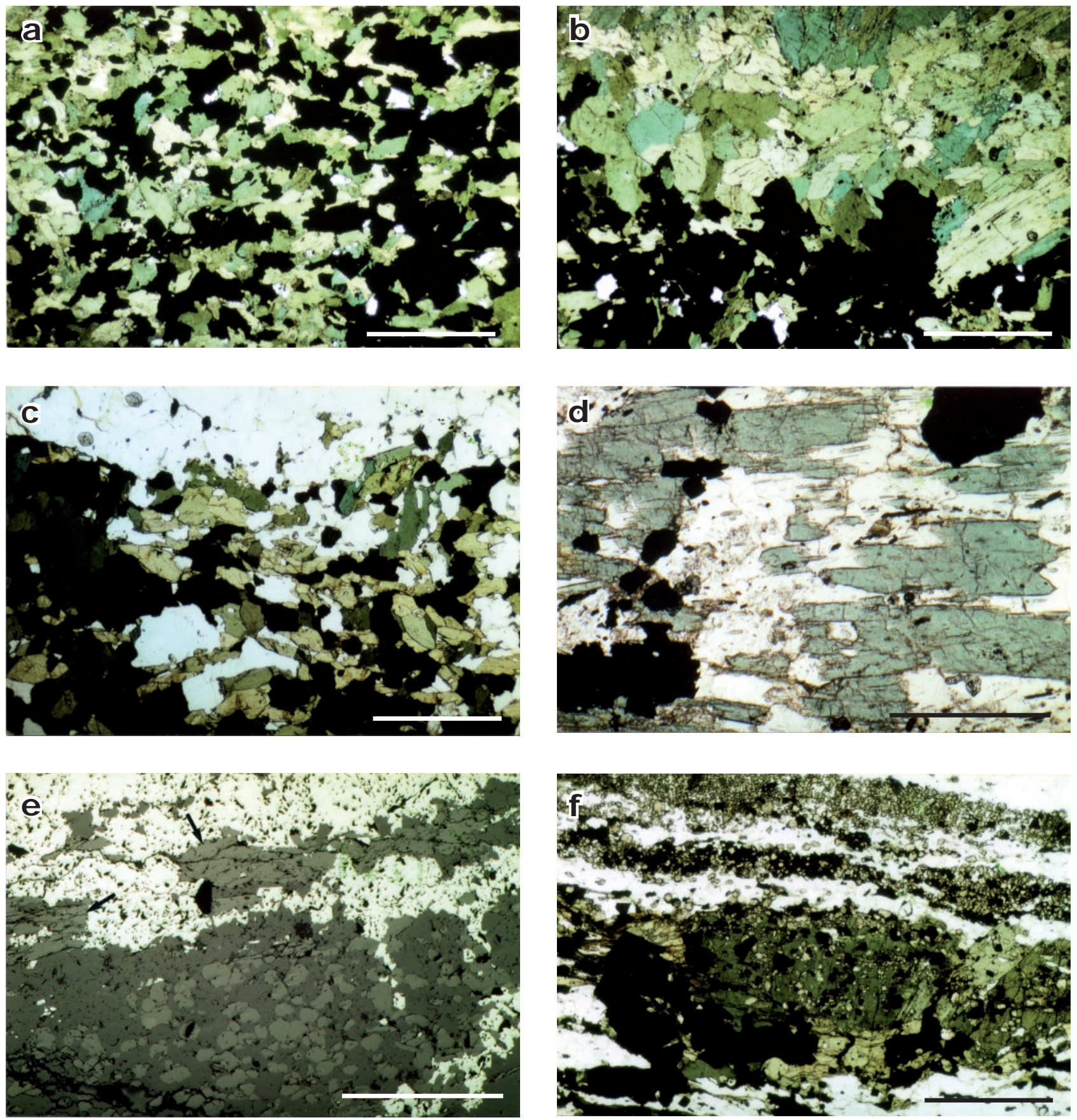

Fig. 12a - Rock containing magnetite (black), amphibole (green) and subordinate quartz (white). Note the irregular grain boundaries of magnetite. Transmitted light, scale bar $1 \mathrm{~mm}$, Sylvani; $\mathbf{b}$ - Contact between magnetite-amphibole rock and hornblendite. Transmitted light, scale bar 1 $\mathrm{mm}$, Sylvani; $\mathbf{c}$ - Contact between bands consisting of magnetite-amphibole rock (lower part) and quartz (mainly upper part). Note the corroded grain boundaries of amphibole in contact to quartz. Transmitted light, scale bar $1 \mathrm{~mm}$, Rudná hora; d - Mineral assemblage of epidote (grey), quartz (white) and magnetite (black). Epidote has corroded grain boundaries and replaces amphibole. Transmitted light, scale bar $1 \mathrm{~mm}$, Kosaře; e - Aggregates of magnetite (bright) and amphibole (arrows) in contact to quartz (slightly darker) that contains abundant rounded apatite crystals. Reflected light, scale bar $1 \mathrm{~mm}$, Kosaře; $\mathbf{f}$ - Interlayered bands consisting either of tiny garnet crystals (garnetite) or magnetite-amphibole rock. The two are separated from one another by small quartz bands (white). In a small contact zone the garnet crystals are overgrown by amphibole. Transmitted light, scale bar $1 \mathrm{~mm}$, Švagrov. 

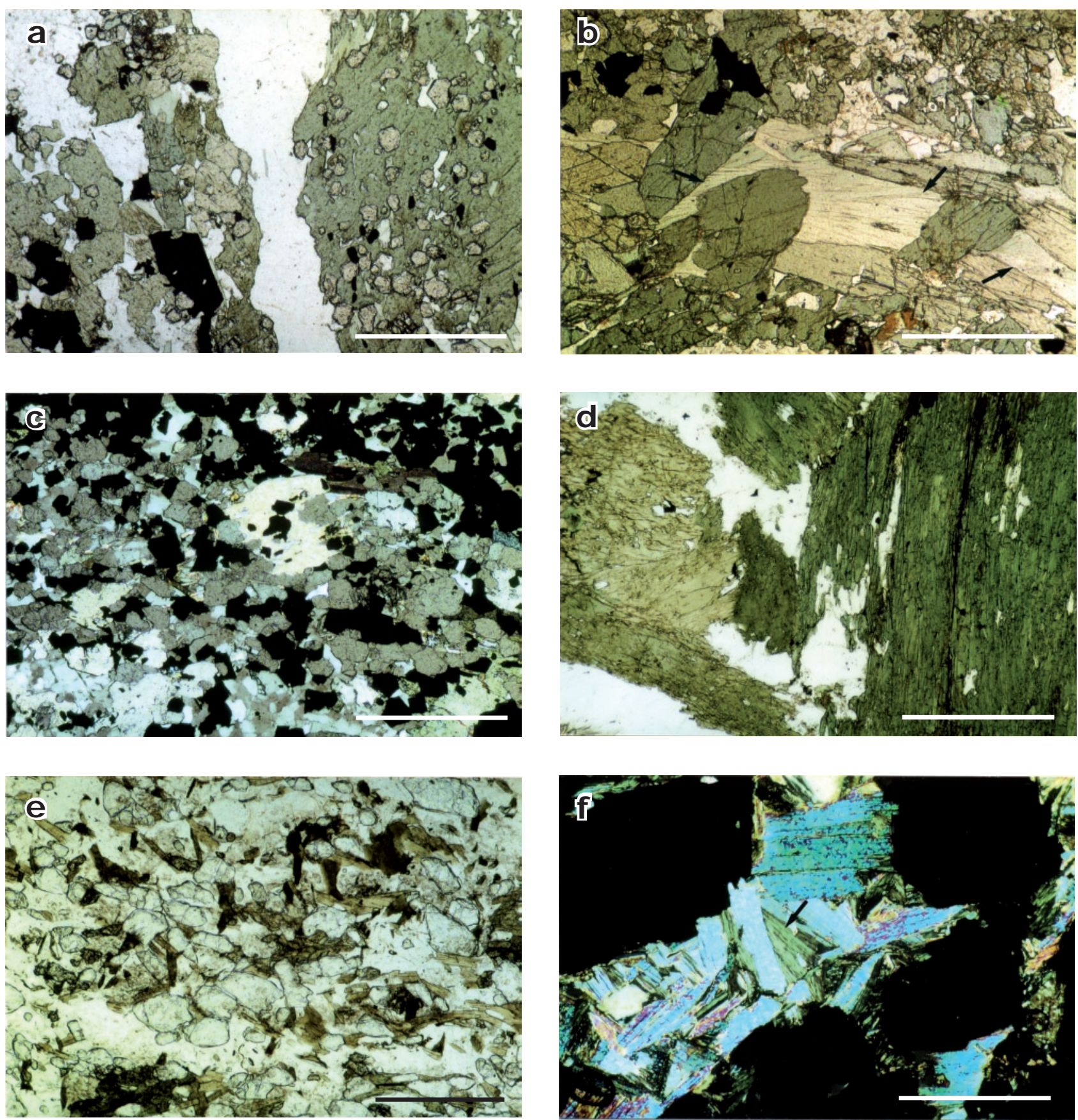

Fig. 13a - Tiny garnet crystals overgrown by magnetite (black) and in particular by amphibole, which is erratically replaced by quartz (white). Transmitted light, scale bar $0.5 \mathrm{~mm}$, Švagrov; $\mathbf{b}$ - Amphibole partially replaced by chlorite (arrows). Magnetite is black, quartz (fine-grained) is the lightest mineral. Transmitted light, scale bar $0.5 \mathrm{~mm}$, Švagrov; c - Crystals of magnetite (black) and apatite (grey) in quartz (light) associated with subordinate proportions of amphibole and epidote grains which are both corroded. Transmitted light, crossed polars, scale bar $0.5 \mathrm{~mm}$, Švagrov; d - Hornblendite, in which the amphibole crystals are partially replaced by quartz. Transmitted light, scale bar $0.5 \mathrm{~mm}$, Švagrov; e - Rock consisting of amphibole (note the corroded grain boundaries), chlorite with biotite (both elongated), abundant rounded apatite crystals and quartz (in the form of the "groundmass"). Transmitted light, scale bar $1 \mathrm{~mm}$, Švagrov; $\mathbf{f}$ - Compact magnetite (black) and biotite, the latter being partially replaced by chlorite (arrow). Transmitted light, scale bar $0.5 \mathrm{~mm}$, Kyzový důl.

and amphibole are preserved. Magnetite, in part slightly martitized, is closely associated with ilmenite, which is mostly concentrated along the magnetite rims. Locally, apatite (up to $0.4 \mathrm{~mm}$ ) is enriched to up to $20 \mathrm{vol}$ \%
(Fig. 13c). In quartz-rich magnetite-amphibole rocks, amphibole is often completely replaced by epidote or is preserved only in relics. Other rock constituents are biotite, chlorite, epidote, and carbonate. 
Metahornblendite contains subordinate magnetite $(<<1$ vol. \%) and varying quantities of quartz, which may replace amphibole (Fig. 13d). Other rock constituents are biotite, feldspar, chlorite, epidote, carbonate, ilmenite and apatite. In places, metahornblendite is rich in quartz with apatite crystals locally abundant (Fig. 13e).

\subsubsection{Břidličná, Kyzový důl and Mnišské jámy}

The magnetite-rich bands consist of predominating magnetite (50 to 90 vol. \%), chlorite, and locally also biotite [magnetite-chlorite-(biotite) rocks]. The magnetite grains (including those which seem to be broken) are embedded in a chlorite groundmass that may contain relics of biotite. Magnetite, chlorite and biotite are associated with varying quantities of albite, epidote, carbonate and ilmenite, while amphibole is absent. Břidličná and Mnišské jámy are dominated by magnetite and chlorite carrying sporadically relics of biotite and newly formed epidote. Kyzový důl contains, at least locally, relatively high amounts of biotite (ratio chlorite : biotite about $2: 1$ to $3: 1$ ) (Fig. 13f) and locally abundant carbonate and plagioclase (Fig. 14a). Plagioclase, containing up to $12.8 \mathrm{~mol}$. \% An, is relatively coarse-grained (up to 2 $\mathrm{mm}$ ). Along the contact of the magnetite-chlorite-(biotite) rocks and quartz bands, chlorite and in part also magnetite are replaced or corroded by quartz (Fig. 14b), whereas the quartz bands may contain relics of chlorite and inclusions of magnetite. In domains with a high carbonate content, carbonate is replaced by quartz (Fig. 14c) carrying inclusions of biotite and chlorite (Fig. 14d).

The magnetite crystals, weakly martitized at places, are mainly concentrated in aggregates of nearly idioblastic grains with smooth, but irregular grain boundaries. The grain size varies between 0.01 and $1.5 \mathrm{~mm}$. The biggest crystals were observed at Břidličná (up to $7 \mathrm{~mm}$ in diameter) and the smallest at Mnišské jámy. Ilmenite occurs mainly along grain boundaries of magnetite in all the three deposits. The elongated ilmenite crystals vary between 0.03 and $0.06 \mathrm{~mm}$. Ilmenite may contain hematite exsolutions (visible in oil only) and may be replaced by titanite (Mnišské jámy) or by blitz-textured hematiterutile intergrowths.

Garnetite of Mnišské jámy occurrs as schlieren-like "bands" alternating with quartz bands and contains, apart from garnet (about 30 to $50 \mathrm{vol} . \%$; 0.1 to $0.4 \mathrm{~mm}$; on average $0.2 \mathrm{~mm}$ ), chlorite, magnetite (both about 10 to 15 vol. \%) and quartz. Within the quartz bands, garnet grains (up to 10 to 15 vol. \%) and tiny chlorite relics (about 1 vol. \%) are sporadically distributed (Fig. 14e). The contact of garnetite with the host rock gneiss is not so sharp; the transition zone is characterized by varying abundance of garnet crystals, decreasing towards the gneiss. In Kyzový důl, garnet crystals were only detected by microscopic investigations. Its single crystals (about $0.1 \mathrm{~mm}$ ) or aggregates are often corroded and widely scattered in quartz, similar to Mnišské jámy (Fig. 14e).

\subsubsection{Zadní Hutisko}

The garnet crystals of garnetite are fine-grained $(0.005$ to $0.04 \mathrm{~mm}$, in maximum to $0.1 \mathrm{~mm}$ ) and occur together with tiny magnetite crystals $(0.06 \mathrm{~mm} ; 2$ to 5 vol. \%), pyrite, $\mathrm{K}$-feldspar and ilmenite $(<1$ vol. \%); the last is often replaced by titanite. These minerals are set in a quartz groundmass. Along the contact of garnetite with magnetite-biotite-(chlorite) rock, garnet crystals are overgrown by biotite and magnetite (Fig. 14f). At the contact with quartz bands, the garnet crystals are bigger and strongly corroded (Fig. 15a).

In a few rock samples were found bands consisting of coarse-grained magnetite and amphibole, with magnetite 0.1 to $0.4 \mathrm{~mm}$ and amphibole 0.2 to $0.6 \mathrm{~mm}$ across (Fig. 15b). The magnetite-amphibole bands are often intersected by subparallel quartz bands. A sample $4 \mathrm{~cm}$ wide contains alternating 12 quartz bands and 11 magnetite-amphibole bands, each 1 to $2 \mathrm{~mm}$ thick. The contacts are characterized by corrosion and replacement of amphibole and, to a lesser degree, of magnetite by quartz (Fig. 15c). Within the quartz are preserved relics of magnetite and amphibole. Magnetite-amphibole rocks may also occur in close contact with garnetite. Along a small zone of the contact, garnet crystals are overgrown by amphibole and magnetite (Fig. 15d). In rare cases, the contacts between these two rock types are sharp and nearly free of overgrowths around garnet (Fig. 15e).

The amphibole-rich rock consists predominantly of amphibole (30 to 70 vol. \%), albite, epidote (the latter two minerals: 25 to 65 vol. \%) and magnetite. Other rock constituents are biotite, and rarely titanite, with carbonate. Amphibole is aligned, coarse-grained (up to $3 \mathrm{~mm}$ ), and broken parallel to its cleavage. The broken crystals are embedded in a groundmass consisting of a mixture of medium-sized epidote (rounded grains are 0.05 to $0.1 \mathrm{~mm}$ across; elongated crystals up to $0.4 \mathrm{~mm}$ ) with fine-grained albite (Fig. 15f). The contacts between epidote/albite and amphibole are corroded as a sign that amphibole was replaced by albite. Magnetite occurs in euhedral to subhedral crystals $(0.05$ to $0.5 \mathrm{~mm}$; on average $0.1 \mathrm{~mm})$, which are included mainly in amphibole, but also in epidote and albite. Biotite (up to $0.2 \mathrm{~mm}$ ) appears in close association with amphibole (Fig. 15f).

Amphibole-rich rocks that contain more than 50 vol. \% plagioclase are known as amphibole gneiss. However, by both macroscopic and microscopic observations the replacement of amphibole by albite and 

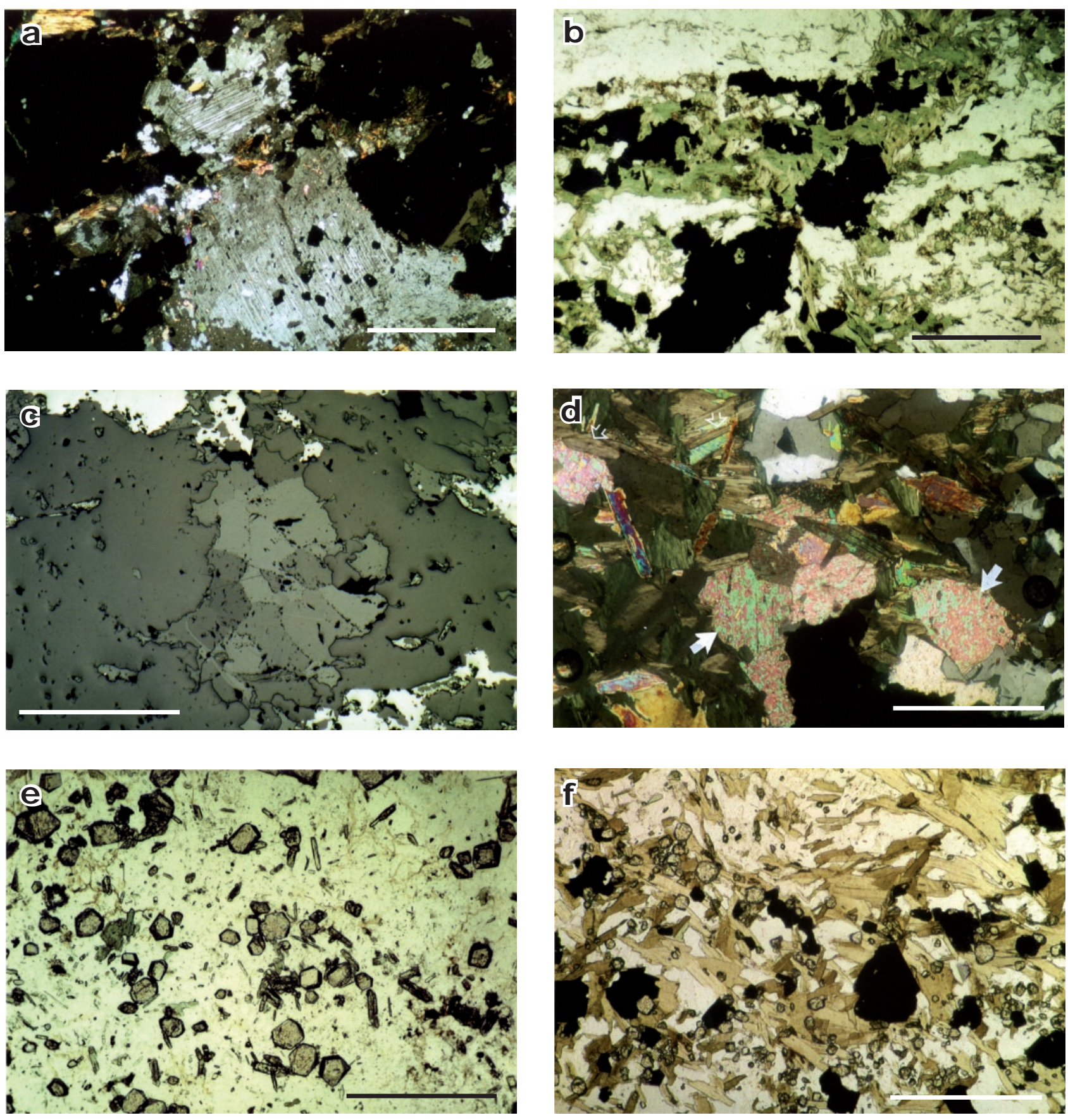

Fig. 14a - Large poikilitic albite blasts in association with magnetite (black), biotite and chlorite. Transmitted light, crossed polars, scale bar $1 \mathrm{~mm}$, Kyzový důl; $\mathbf{b}$ - Layer of magnetite-chlorite in association with quartz. Note the corroded grain boundaries of chlorite and magnetite in contact to quartz. Transmitted light, scale bar $1 \mathrm{~mm}$. Kyzový důl; c - Inclusion of polycrystalline carbonate (anisotropic; in the centre) enclosed by quartz. Note the corroded grain boundaries of carbonate. Magnetite is bright and also corroded by quartz. Reflected light, scale bar 1 mm, Kyzový důl; d - Assemblage of magnetite (black), carbonate (arrows), biotite, chlorite (open arrows) and quartz. Transmitted light, scale bar $0.5 \mathrm{~mm}$, Kyzový důl; e - Irregularly distributed garnet crystals within quartz. Needle-like grains are chlorite remnants. Transmitted light, scale bar 0.5 mm, Mnišské jámy; $\mathbf{f}$ - Assemblage of magnetite and biotite, which contains inclusions of garnet crystals. Note that biotite has corroded grain boundaries in contact to quartz. Transmitted light, scale bar $0.5 \mathrm{~mm}$, Zadní Hutisko. 

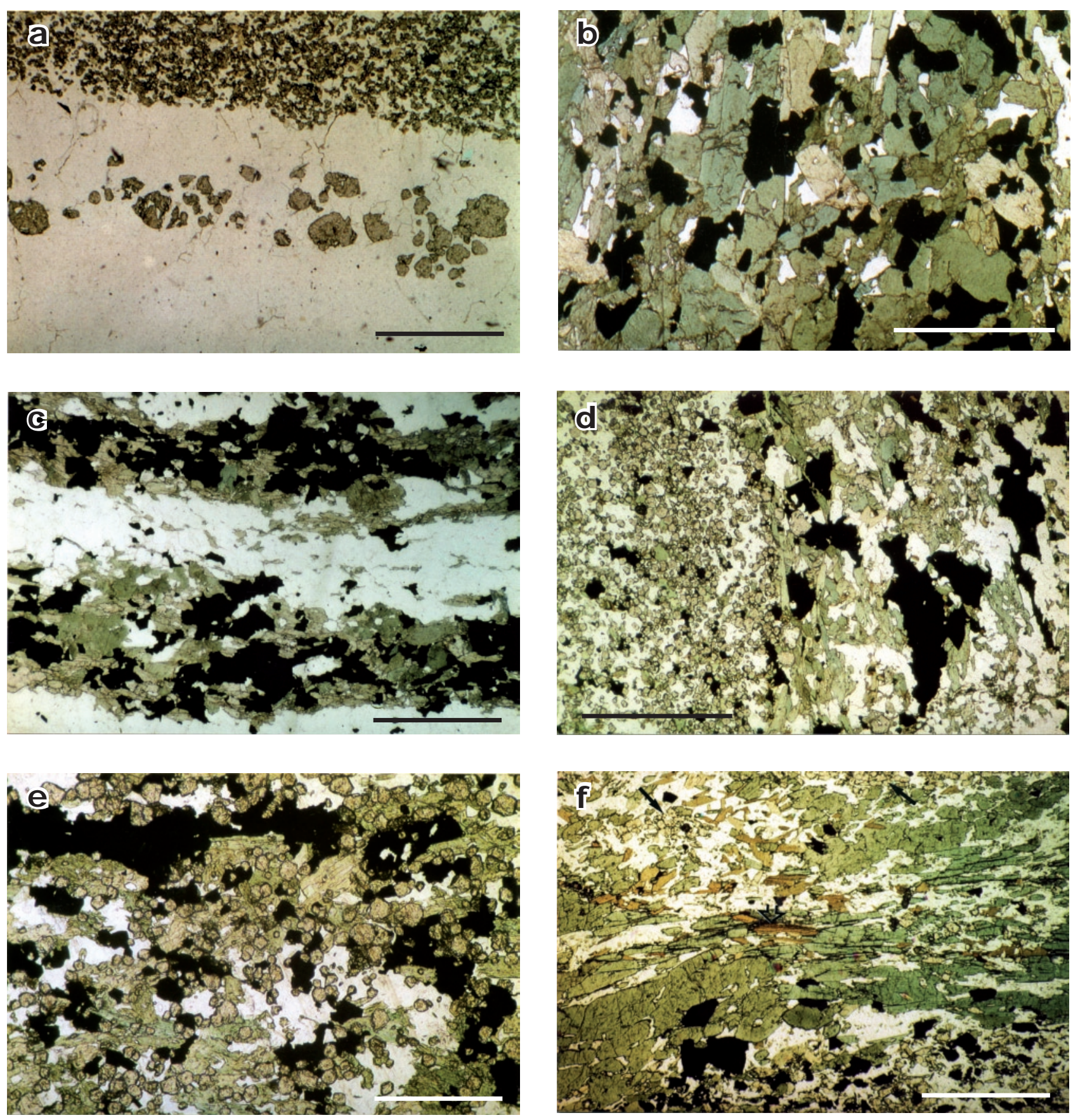

Fig. 15a - Contact of tiny garnet crystals embedded in a quartz groundmass (= garnetite; upper part) and quartz of another generation. The latter contains large and corroded garnet crystals arranged parallel to the contact. Transmitted light, scale bar $1 \mathrm{~mm}$, Zadní Hutisko; b - Magnetite-amphibole rock in association with quartz occurring predominantly along grain boundaries of corroded amphibole crystals. Transmitted light, scale bar $1 \mathrm{~mm}$, Zadní Hutisko; c - Alternate succession of magnetite-amphibole and quartz bands. Note the corroded grain boundaries of tiny amphibole and magnetite grains within quartz. Transmitted light, scale bar $1 \mathrm{~mm}$, Zadní Hutisko; d - Contact of fine-grained garnetite (left side) and coarsegrained magnetite-amphibole rock, which contains quartz (note the corroded amphibole grains in contact with this mineral). Transmitted light, scale bar $1 \mathrm{~mm}$, Zadní Hutisko; e - Contact between garnetite and quartz-bearing magnetite-amphibole rock. The garnet crystals are overgrown by amphibole and magnetite. Transmitted light, scale bar $0.5 \mathrm{~mm}$, Zadní Hutisko; $\mathbf{f}$ - Elongated amphibole grains broken parallel to their cleavage and embedded in a mixture of albite and epidote (arrows). Closely associated are elongated biotite grains (open arrow). Magnetite is black. Note the corroded outlines of amphibole. Transmitted light, scale bar $1 \mathrm{~mm}$, Zadní Hutisko. 
epidote is easily recognizable and therefore the rock can be termed as strongly albitized and epidotized metahornblendite.

\section{Mineral chemistry}

Main or significant minerals of the investigated rocks are amphibole, chlorite, biotite, garnet, quartz, magnetite, ilmenite and apatite. Other minerals are feldspar, carbonate and epidote. The relative abundance of these minerals of the iron-rich mineralizations and the associated rocks within the eight locations is variable and summarized in Tab. 1 .

Amphibole compositions, selection of which is presented in the Tables 2 and 3, are plotted in the classification diagrams after Leake et al. (1997) in Fig. 16 (open symbols: iron-rich rocks and garnetites; filled symbols: metahornblendite). Amphiboles occur in:
1. magnetite-amphibole rocks [Rudná hora, I and II; Sylvani, III to V; Kosaře, VI to VIII; Švagrov, IX to XI; and Zadní Hutisko, XII and XIII] (Tab. 2);

2. rocks with accessory magnetite [metahornblendite: Rudná hora (I and II), Švagrov (III to VI) and Zadní Hutisko (VII); actinolite-chlorite schist and chloritehornblende-epidote rock of the Sobotín Massif (VIII and IX), analyses are from Fiala et al. 1980; and rocks of basic dykes from Zámčisko (X and XI), analyses are from Fojt et al. 1997] (Tab. 3).

A great majority of amphibole analyses (about $75 \%$ ) have $\mathrm{Mg} /(\mathrm{Mg}+\mathrm{Fe})$ ratios 0.6 and 0.8 and $\mathrm{Si}$ values 6 to 6.8 , corresponding to tschermakite or magnesiohornblende (Fig. 16). Nine are magnesiohornblende with distinctly higher Si values (up to 7.5), whereas five represent actinolite and three analyses from Zámčisko correspond to ferrohornblende.

Selected biotite analyses are presented in Tab. 4. The data, which are summarized in Fig. 17 (diagram after

Tab. 1 Relative abundances of minerals $($ amphibole $=$ Amph; biotite $=$ Bio; quartz $=$ Qtz; epidote $=$ Ep; feldspar $=$ Fsp; Carbonate $=$ Car; magne tite $=$ Mag; ilmenite $=\mathrm{Il}$; and apatite $=\mathrm{Ap})$ and of garnetite $($ consisting of spessartite-rich garnet $)=$ Garn with meta-hornblendite $(\mathrm{M}-\mathrm{hb}): \bullet$ not occurring, $\bigcirc$ in traces, $\square$ moderate; $\square \square$ frequent; $\square \square \square$ abundant.

\begin{tabular}{|c|c|c|c|c|c|c|c|c|c|c|c|c|}
\hline & \multicolumn{10}{|c|}{ Minerals and their abundance in iron-rich rocks } & \multicolumn{2}{|c|}{ Associated rocks } \\
\hline & Amph & Bio & Qtz & Chl & Ep & Fsp & Car & Mag & Ilm & Ap & Garn & M-hb \\
\hline Rudná hora & $\square \square \square$ & $\bullet$ & •-口पव & $\circ$ & $\circ$ & $\bullet$ & $\bullet$ & $\square \square \square$ & 0 & $\circ$ & $\bullet$ & $\square \square \square$ \\
\hline Sylvani & $\square \square \square$ & $\square$ & •-वप् & $\bullet$ & ० & $\bullet$ & $\square$ & $\square \square \square$ & $\square$ & ० & $\bullet$ & $\square \square \square$ \\
\hline Kosaře & $\square$ & $\bullet$ & $\square \square \square$ & $\square$ & o & o & $\square$ & $\square \square \square$ & ० & $\square$ & $\square$ & $\bullet$ \\
\hline Švagrov & $\square-\square \square \square$ & $\square$ & $\square-\square \square \square$ & $\square$ & $\circ$ & $\bullet$ & $\square$ & प्व & ○ & $\square \square$ & $\square \square$ & $\square \square$ \\
\hline Zadní Hutisko & $\square$ & $\square \square \square$ & $\square \square \square$ & $\circ$ & $\bullet$ & $\circ$ & $\square$ & $\square-\square \square$ & ० & $\circ$ & $\square \square \square$ & $\square$ \\
\hline Kyzový důl & $\bullet$ & $\square \square$ & प-वप् & $\square \square$ & ० & $\square \square$ & $\square \square$ & वप् & ० & ० & $\circ$ & $\bullet$ \\
\hline Břidličná & $\bullet$ & $\square$ & 口-वप् & पव口 & $\square$ & $\square$ & $\square$ & $\square \square \square$ & ○ & ० & $\bullet$ & $\bullet$ \\
\hline Mnišské jámy & $\bullet$ & $\square$ & प-वप् & $\square \square \square$ & $\square$ & 0 & $\square$ & $\square \square \square$ & ○ & $\square$ & $\square$ & $\bullet$ \\
\hline
\end{tabular}

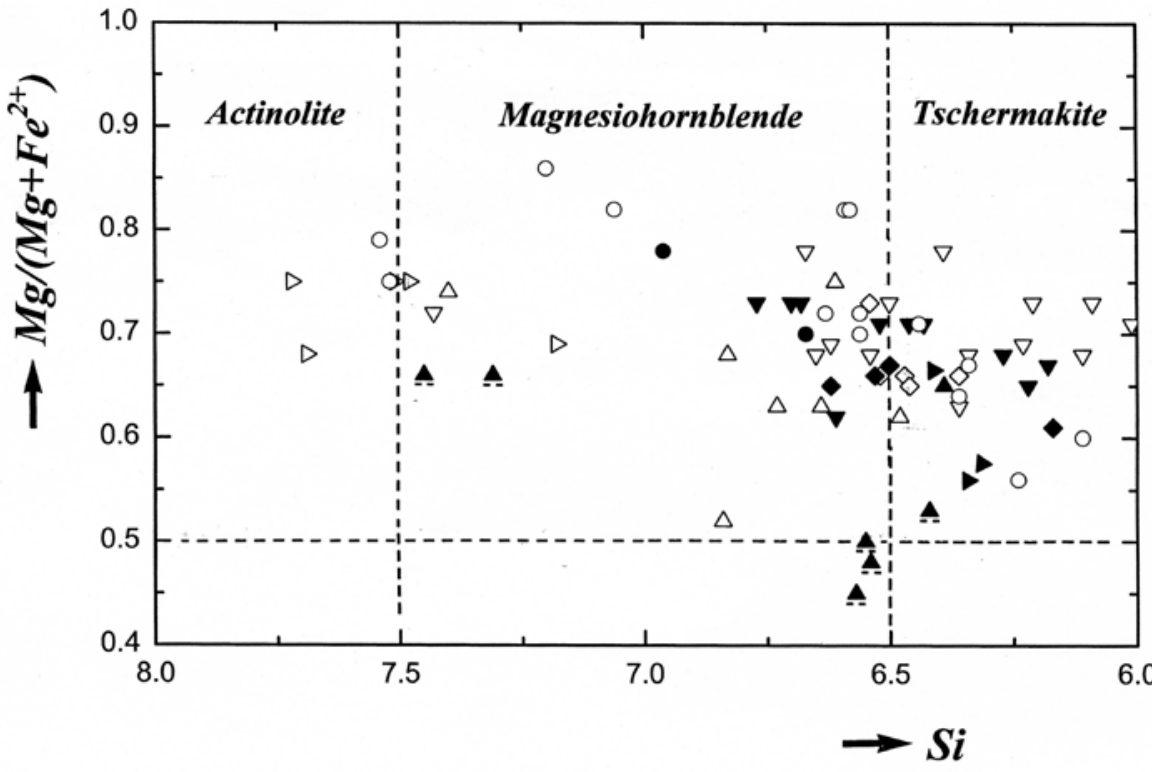

\begin{tabular}{|c|l|c|}
\hline & Sobotin Massif & $\bullet$ \\
\hline$\triangle$ & Sylvani & $\boldsymbol{\Delta}$ \\
\hline$\diamond$ & Rudná hora & $\bullet$ \\
\hline$\bigcirc$ & Kosaře & \\
\hline$\nabla$ & Švagrov & $\nabla$ \\
\hline$\triangleright$ & Zadni Hutisko & \\
\hline & Záméisko & $\boldsymbol{\Delta}$ \\
\hline
\end{tabular}

Fig. 16 Amphibole classification diagram $\mathrm{Mg} /\left(\mathrm{Mg}+\mathrm{Fe}^{2+}\right)$ versus $\mathrm{Si}$ (apfu) according to Leake et al. (1997). Diagram is valid for amphiboles with $\mathrm{Ca}_{\mathrm{B}} \geq 1.50$ and $(\mathrm{Na}+\mathrm{K})_{\mathrm{A}}<0.50$. 
Tab. 2 Selected amphibole analyses of magnetite-amphibole rocks [Rudná hora (I and II), Sylvani (III to V), Kosaře (VI to VIII), Švagrov (IX to XI) and Zadní Hutisko, XII and XIII)] in wt. \% (upper part) and apfu (lower part).

\begin{tabular}{|c|c|c|c|c|c|c|c|c|c|c|c|c|c|}
\hline & \multicolumn{2}{|c|}{ Rudná hora } & \multicolumn{3}{|c|}{ Sylvani } & \multicolumn{3}{|c|}{ Kosaře } & \multicolumn{3}{|c|}{ Švagrov } & \multicolumn{2}{|c|}{ Zadní Hutisko } \\
\hline & I & II & III & IV & $\mathbf{V}$ & VI & VII & VIII & IX & $\mathrm{X}$ & XI & XII & XIII \\
\hline $\mathrm{SiO}_{2}$ & 42.23 & 45.80 & 43.31 & 45.58 & 52.85 & 40.70 & 45.11 & 52.43 & 41.05 & 44.63 & 51.16 & 49.51 & 54.12 \\
\hline $\mathrm{TiO}_{2}$ & 0.16 & 0.15 & 0.49 & 0.32 & 0.06 & 0.31 & 0.24 & n.d. & 0.31 & 0.45 & 0.13 & 0.21 & n.d. \\
\hline $\mathrm{Al}_{2} \mathrm{O}_{3}$ & 11.78 & 11.15 & 10.11 & 8.83 & 3.36 & 14.70 & 11.17 & 3.90 & 15.74 & 10.60 & 4.24 & 6.46 & 2.33 \\
\hline $\mathrm{FeO}_{\text {anal }}$ & 17.49 & 15.76 & 19.06 & 17.94 & 11.80 & 17.19 & 15.74 & 12.09 & 16.74 & 17.14 & 13.05 & 14.18 & 11.12 \\
\hline $\mathrm{MnO}$ & 0.36 & 0.41 & 0.64 & 0.78 & 0.90 & 0.75 & 0.75 & 0.32 & 0.65 & 0.60 & 0.59 & 1.24 & 1.46 \\
\hline $\mathrm{MgO}$ & 10.72 & 12.28 & 9.59 & 10.85 & 16.48 & 8.92 & 11.41 & 16.63 & 9.87 & 11.13 & 14.45 & 12.94 & 16.91 \\
\hline $\mathrm{CaO}$ & 12.18 & 11.86 & 11.96 & 11.79 & 10.99 & 11.99 & 11.89 & 10.70 & 11.37 & 11.86 & 12.88 & 12.38 & 11.60 \\
\hline $\mathrm{Na}_{2} \mathrm{O}$ & 1.08 & 0.97 & 1.17 & 1.15 & 0.53 & 1.26 & 0.89 & 0.95 & 1.22 & 0.75 & 0.35 & 0.65 & 0.12 \\
\hline $\mathrm{K}_{2} \mathrm{O}$ & 0.30 & 0.23 & 0.38 & 0.26 & 0.11 & 0.34 & 0.17 & 0.09 & 0.22 & 0.14 & 0.04 & 0.13 & 0.09 \\
\hline $\mathrm{FeO}_{\text {calc }}$ & 9.77 & 8.31 & 12.78 & 11.22 & 10.13 & 9.15 & 8.57 & 9.74 & 7.33 & 9.31 & 9.85 & 10.35 & 9.87 \\
\hline $\mathrm{Fe}_{2} \mathrm{O}_{3 \text { calc }}$ & 8.58 & 8.28 & 6.98 & 7.46 & 1.85 & 8.93 & 7.97 & 2.61 & 10.46 & 8.70 & 3.55 & 4.19 & 1.39 \\
\hline $\mathrm{H}_{2} \mathrm{O}_{\text {calc }}$ & 2.03 & 2.09 & 2.05 & 2.03 & 2.08 & 1.99 & 2.05 & 2.09 & 2.05 & 2.04 & 2.06 & 2.06 & 2.10 \\
\hline total & 100.19 & 101.53 & 99.46 & 100.27 & 99.34 & 99.04 & 100.22 & 99.46 & 100.27 & 100.21 & 99.30 & 100.18 & 99.99 \\
\hline $\mathrm{Si}$ & 6.375 & 6.572 & 6.518 & 6.728 & 7.598 & 6.112 & 6.578 & 7.520 & 6.010 & 6.542 & 7.425 & 7.184 & 7.720 \\
\hline $\mathrm{Al}$ & 1.625 & 1.428 & 1.482 & 1.272 & 0.402 & 1.888 & 1.422 & 0.480 & 1.990 & 1.458 & 0.575 & 0.816 & .280 \\
\hline total & 8.000 & 8.000 & 8.000 & 8.000 & 8.000 & 2.000 & 8.000 & 8.000 & 8.000 & 8.000 & 8.000 & 8.000 & 8.000 \\
\hline $\mathrm{Ti}$ & 0.018 & 0.016 & 0.055 & 0.036 & 0.007 & 0.035 & 0.026 & - & 0.034 & 0.049 & 0.014 & 0.023 & - \\
\hline $\mathrm{Al}$ & 0.423 & 0.458 & 0.312 & 0.264 & 0.167 & 0.713 & 0.498 & 0.179 & 0.726 & 0.373 & 0.151 & 0.289 & 0.112 \\
\hline $\mathrm{Fe}^{3+}$ & 0.952 & 0.894 & 0.791 & 0.829 & 0.200 & 0.815 & 0.875 & 0.282 & 1.152 & 0.960 & 0.388 & 0.457 & 0.149 \\
\hline $\mathrm{Mg}$ & 2.357 & 2.626 & 2.151 & 2.388 & 3.531 & 1.997 & 2.480 & 3.555 & 2.154 & 2.432 & 3.127 & 2.799 & 3.596 \\
\hline $\mathrm{Fe}^{2+}$ & 1.205 & 0.998 & 1.608 & 1.385 & 1.095 & 1.344 & 1.045 & 0.984 & 0.898 & 1.142 & 1.196 & 1.263 & 1.143 \\
\hline $\mathrm{Mn}$ & 0.045 & 0.008 & 0.082 & 0.098 & - & 0.096 & 0.076 & - & 0.036 & 0.044 & 0.072 & 0.153 & - \\
\hline total & 5.000 & 5.000 & 4.999 & 5.000 & 5.000 & 5.000 & 5.000 & 5.000 & 5.000 & 5.000 & 5.000 & 5.000 & 5.000 \\
\hline $\mathrm{Fe}^{2+}$ & - & - & - & - & 0.123 & - & - & 0.185 & - & - & - & - & 0.035 \\
\hline $\mathrm{Mn}$ & - & 0.042 & - & - & 0.110 & - & 0.017 & 0.039 & 0.045 & 0.031 & - & - & 0.177 \\
\hline $\mathrm{Ca}$ & 1.925 & 1.824 & 1.929 & 1.864 & 1.694 & 1.929 & 1.840 & 1.645 & 1.783 & 1.863 & 2.003 & 1.925 & 1.773 \\
\hline $\mathrm{Na}$ & 0.075 & 0.134 & 0.071 & 0.136 & 0.073 & 0.071 & 0.143 & 0.131 & 0.172 & 0.106 & - & 0.075 & 0.015 \\
\hline total & 2.000 & 2.000 & 2.000 & 2.000 & 2.000 & 2.000 & 2.000 & 2.000 & 2.000 & 2.000 & 2.003 & 2.000 & 2.000 \\
\hline $\mathrm{Na}$ & 0.234 & 0.136 & 0.269 & 0.193 & 0.074 & 0.296 & 0.108 & 0.134 & 0.175 & 0.107 & 0.099 & 0.108 & 0.018 \\
\hline $\mathrm{K}$ & 0.057 & 0.042 & 0.073 & 0.050 & 0.020 & 0.065 & 0.032 & 0.016 & 0.041 & 0.026 & 0.007 & 0.024 & 0.016 \\
\hline total & 0.291 & 0.178 & 0.342 & 0.243 & 0.094 & 0.361 & 0.140 & 0.150 & 0.216 & 0.133 & 0.106 & 0.132 & 0.034 \\
\hline
\end{tabular}

Siderophyllite $\mathrm{KFe}_{2.5} \mathrm{Al} \boldsymbol{l}_{0.5}\left[(\mathrm{OH})_{2} / \mathrm{Al}_{1.5} \mathrm{Si}_{2.5} \mathrm{O}_{10}\right]$
Eastonite $\mathrm{KMg}_{2.5} \mathrm{Al}_{0.5}\left[(\mathrm{OH})_{2} / \mathrm{Al}_{1.5} \mathrm{Si}_{2.5} \mathrm{O}_{10}\right]$

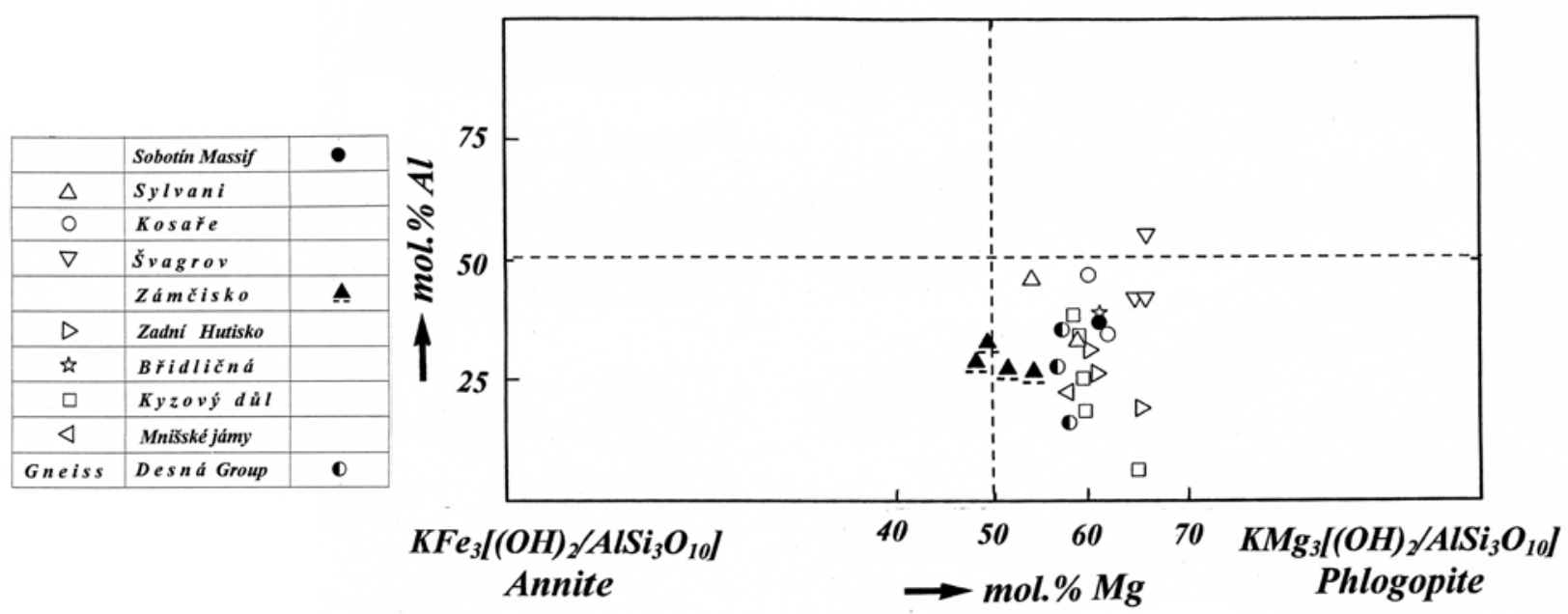

Fig. 17 Biotite classification diagram according to Rieder et al. (1998). 
Tab. 3 Selected amphibole analyses of hornblendite from Rudná hora (I and II), Švagrov (III to VI) and Zadní Hutisko (Z. H.) (VII); chlorite-hornblende-epidote rock (VIII) and actinolite-chlorite schist (IX) of the Sobotín Massif (analyses of Fiala et al. 1980); rocks from basic dyke of Zámčisko (X and XI; analyses Fojt et al. 1997), and Lahn-Dill type samples of the Malý Děd Mt. (XII to XIV). In wt. \% (upper part) and apfu (lower part).

\begin{tabular}{|c|c|c|c|c|c|c|c|c|c|c|c|c|c|c|}
\hline & \multicolumn{2}{|c|}{ Rudná hora } & \multicolumn{4}{|c|}{ Švagrov } & \multirow{2}{*}{$\begin{array}{c}\text { Z. H. } \\
\text { VII }\end{array}$} & \multicolumn{2}{|c|}{ Sobotín } & \multicolumn{2}{|c|}{ Zámčisko } & \multicolumn{3}{|c|}{ Malý Děd } \\
\hline & I & II & III & IV & V & VI & & VIII & IX & $\mathrm{X}$ & XI & XII & XIII & XIV \\
\hline $\mathrm{SiO}_{2}$ & 41.39 & 45.15 & 42.85 & 47.55 & 42.30 & 46.07 & 43.98 & 45.42 & 55.67 & 43.33 & 50.33 & 39.88 & 48.03 & 49.46 \\
\hline $\mathrm{TiO}_{2}$ & 0.48 & 0.47 & 0.37 & 0.33 & 0.42 & 0.32 & 0.38 & 0.38 & 0.08 & 0.36 & 0.29 & 0.05 & n.d. & n.d. \\
\hline $\mathrm{Al}_{2} \mathrm{O}_{3}$ & 14.09 & 10.44 & 15.92 & 10.76 & 15.34 & 11.18 & 12.41 & 10.18 & 1.94 & 12.41 & 5.08 & 18.19 & 3.64 & 1.57 \\
\hline $\mathrm{V}_{2} \mathrm{O}_{3}$ & 0.01 & n.d. & 0.10 & 0.06 & 0.06 & n.d. & n.a. & n.a. & n.a. & n.a. & n.a. & n.d. & n.d. & n.d. \\
\hline $\mathrm{Cr}_{2} \mathrm{O}_{3}$ & 0.01 & n.d. & 0.03 & 0.02 & n.d. & n.d. & n.a. & n.a. & n.a. & n.a. & n.a. & n.d. & n.d. & n.d. \\
\hline $\mathrm{FeO}_{\text {anal }}$ & 15.95 & 15.38 & 15.19 & 13.50 & 15.20 & 13.23 & 17.36 & 14.78 & 5.81 & 19.11 & 15.60 & 25.30 & 37.74 & 38.66 \\
\hline $\mathrm{MnO}$ & 0.24 & 0.26 & 0.32 & 0.28 & 0.39 & 0.42 & 0.22 & 0.24 & 0.18 & 0.29 & 0.24 & 0.26 & 0.62 & 0.74 \\
\hline $\mathrm{MgO}$ & 10.26 & 11.91 & 9.87 & 13.21 & 9.70 & 12.39 & 10.35 & 12.14 & 20.86 & 8.90 & 13.27 & 2.02 & 5.31 & 6.10 \\
\hline $\mathrm{CaO}$ & 12.18 & 12.46 & 11.80 & 12.03 & 12.16 & 12.10 & 11.78 & 11.62 & 12.47 & 11.45 & 12.23 & 10.40 & 1.96 & 1.18 \\
\hline $\mathrm{Na}_{2} \mathrm{O}$ & 1.57 & 1.15 & 1.14 & 0.82 & 1.40 & 1.17 & 1.27 & 1.48 & 0.28 & 1.83 & 0.65 & 1.13 & 0.19 & 0.06 \\
\hline $\mathrm{K}_{2} \mathrm{O}$ & 0.91 & 0.74 & 0.30 & 0.17 & 0.31 & 0.15 & 0.46 & 0.21 & 0.04 & 0.44 & 0.13 & 0.30 & 0.04 & 0.04 \\
\hline $\mathrm{FeO}_{\text {calc }}$ & 11.52 & 11.58 & 9.46 & 9.09 & 8.42 & 8.03 & 10.80 & 9.17 & 4.33 & 13.74 & 12.09 & 21.96 & 37.74 & 38.66 \\
\hline $\mathrm{Fe}_{2} \mathrm{O}_{3 \mathrm{calc}}$ & 4.93 & 4.22 & 6.37 & 4.90 & 7.52 & 5.78 & 7.29 & 6.23 & 1.64 & 5.97 & 3.90 & 3.72 & - & - \\
\hline $\mathrm{H}_{2} \mathrm{O}_{\text {calc }}$ & 2.01 & 2.04 & 2.07 & 2.10 & 2.05 & 2.07 & 2.05 & 2.04 & 2.15 & 2.02 & 2.06 & 1.96 & 1.89 & 1.89 \\
\hline total & 99.60 & 100.42 & 100.60 & 101.32 & 100.09 & 99.68 & 100.26 & 99.11 & 99.64 & 100.74 & 100.27 & 99.87 & 99.42 & 99.70 \\
\hline $\mathrm{Si}$ & 6.170 & 6.621 & 6.215 & 6.769 & 6.183 & 6.679 & 6.422 & 6.668 & 7.744 & 6.420 & 7.309 & 6.106 & 7.630 & 7.841 \\
\hline $\mathrm{Al}$ & 1.830 & 1.379 & 1.785 & 1.231 & 1.817 & 1.321 & 1.578 & 1.332 & 0.256 & 1.580 & 0.691 & 1.894 & 0.370 & 0.159 \\
\hline total & 8.000 & 8.000 & 8.000 & 8.000 & 8.000 & 8.000 & 8.000 & 8.000 & 8.000 & 8.000 & 8.000 & 8.000 & 8.000 & 8.000 \\
\hline $\mathrm{Ti}$ & 0.054 & 0.052 & 0.040 & 0.035 & 0.047 & 0.035 & 0.042 & 0.043 & 0.008 & 0.040 & 0.031 & 0.006 & - & - \\
\hline Al & 0.646 & 0.426 & 0.937 & 0.575 & 0.826 & 0.589 & 0.558 & 0.430 & 0.062 & 0.589 & 0.178 & 1.389 & 0.312 & 0.134 \\
\hline V & 0.001 & - & 0.011 & 0.007 & 0.007 & - & - & - & - & - & - & - & - & - \\
\hline $\mathrm{Cr}$ & 0.001 & - & 0.003 & 0.002 & - & - & - & - & - & - & - & - & - & - \\
\hline $\mathrm{Fe}^{3+}$ & 0.553 & 0.466 & 0.695 & 0.525 & 0.827 & 0.631 & 0.801 & 0.698 & 0.174 & 0.666 & 0.426 & 0.428 & - & - \\
\hline $\mathrm{Mg}$ & 2.280 & 2.603 & 2.134 & 2.803 & 2.113 & 2.678 & 2.253 & 2.657 & 4.326 & 1.966 & 2.873 & 0.461 & 1.257 & 1.441 \\
\hline $\mathrm{Fe}^{2+}$ & 1.436 & 1.421 & 1.148 & 1.053 & 1.031 & 0.973 & 1.319 & 1.142 & 0.430 & 1.702 & 1.468 & 2.716 & 5.014 & 5.126 \\
\hline $\mathrm{Mn}$ & 0.030 & 0.033 & -0.032 & - & 0.048 & 0.051 & 0.027 & 0.030 & - & 0.037 & 0.024 & - & 0.083 & 0.099 \\
\hline total & 5.001 & 5.000 & 5.000 & 5.000 & 4.900 & 4.957 & 5.000 & 5.000 & 5.000 & 5.000 & 5.000 & 5.000 & & \\
\hline $\mathrm{Fe}^{2+}$ & - & - & - & 0.029 & & - & - & - & 0.081 & - & - & 0.095 & & \\
\hline Mn & - & - & 0.007 & 0.033 & & - & - & - & 0.021 & - & 0.006 & 0.034 & & \\
\hline $\mathrm{Ca}$ & 1.946 & 1.958 & 1.834 & 1.835 & 1.904 & 1.880 & 1.844 & 1.828 & 1.859 & 1.818 & 1.903 & 1.706 & 0.334 & 0.200 \\
\hline $\mathrm{Na}$ & 0.054 & 0.042 & 0.159 & 0.103 & 0.096 & 0.120 & 0.156 & 0.172 & 0.039 & 0.182 & 0.091 & 0.165 & - & - \\
\hline total & 2.000 & 2.000 & 2.000 & 2.000 & 2.000 & 2.000 & 2.000 & 2.000 & 2.000 & 2.000 & 2.000 & 2.000 & 7.000 & 7.000 \\
\hline $\mathrm{Na}$ & 0.400 & 0.285 & 0.162 & 0.124 & 0.301 & 0.209 & 0.204 & 0.250 & 0.036 & 0.344 & 0.092 & 0.171 & 0.058 & 0.018 \\
\hline $\mathrm{K}$ & 0.173 & 0.138 & 0.056 & 0.031 & 0.058 & 0.028 & 0.087 & 0.040 & 0.007 & 0.083 & 0.024 & 0.059 & 0.008 & 0.008 \\
\hline total & 0.573 & 0.423 & 0.218 & 0.155 & 0.359 & 0.237 & 0.291 & 0.290 & 0.043 & 0.427 & 0.116 & 0.230 & 0.066 & 0.026 \\
\hline
\end{tabular}

Rieder et al. 1998), were obtained from the following rock samples:

1. magnetite-rich rocks, either associated with amphibole (magnetite-amphibole rocks) or with chlorite [magnetite-chlorite-(biotite) rocks]. Biotite of amphibole-magnetite rocks is from Sylvani (Tab. 4, I) and Kosaře (II), whereas biotite found in the magnetite-chlorite-(biotite) rocks belongs to the occurrences of Břidličná (III), Kyzový důl (IV), Zadní Hutisko (V) and Mnišské jámy (VI);

2. hornblendite from Švagrov (VII), rocks from the basic dyke of Zámčisko (VIII; analysis of Fojt et al. 1997) and the Sobotín Massif (IX; analysis of Fiala et al. 1980);
3 . the host gneiss may contain biotite instead of chlorite or both [Mnišské jámy $(\mathrm{X})$ and Zadní Hutisko (XI)].

The analyses of chlorite are presented in the ternary diagram $\mathrm{Fe}_{\text {tot }}-\mathrm{Mg}-\mathrm{Al}^{\mathrm{VI}}$ proposed by Mücke and Farshad (2005) (Fig. 18) and in diagram $\mathrm{Fe}_{\text {tot }} /\left(\mathrm{Fe}_{\text {tot }}+\mathrm{Mg}\right)-\mathrm{Si}$ according to Melka (1965) (Fig. 19). The selected analyses are shown in Tab. 5. Chlorite occurs in:

1. magnetite-amphibole rocks, in which the chlorite was formed at the expense of amphibole (Fig. 13b). The analyses are from Rudná hora (I and II), Kosaře (III and IV) and Švagrov (V and VI);

2. magnetite-chlorite-(biotite) rocks (Figs 13f, 14a and 14b). The analyses are from Břidličná (VII and VIII), 
Genesis of the magnetite mineralizations in the Silesian Desná Group, Czech Republic

Tab. 4 Selected biotite analyses of magnetite-amphibole rocks [Sylvani (I) and Kosaře (II)], magnetite-chlorite-(biotite) rocks [Břidličná (III), Kyzový důl (IV), Zadní Hutisko (V) and Mnišské jámy (VI)], hornblendite of Švagrov (VII), rocks from basic dyke of Zámčisko [(VIII); analyses of Fojt et al. 1997], albite-epidote amphibolite of the Sobotín Massif [(IX); analyses of Fiala et al. 1980], and gneiss from Mnišské jámy (X) and Zadní Hutisko (XI). In wt. \% (upper part) and apfu (lower part).

\begin{tabular}{|c|c|c|c|c|c|c|c|c|c|c|c|}
\hline & \multicolumn{6}{|c|}{ Magnetite-rich rocks with } & \multicolumn{5}{|c|}{ Rocks with accessory magnetite } \\
\hline & \multicolumn{2}{|c|}{ Amphibole } & \multicolumn{4}{|c|}{ Chlorite-(biotite) } & \multicolumn{3}{|c|}{ Amphibole-rich } & \multicolumn{2}{|c|}{ Gneiss } \\
\hline & $\begin{array}{l}\text { Syl. } \\
\text { I }\end{array}$ & $\begin{array}{c}\text { Kos. } \\
\text { II }\end{array}$ & $\begin{array}{c}\text { Břid. } \\
\text { III }\end{array}$ & $\begin{array}{c}\text { K. důl } \\
\text { IV }\end{array}$ & $\begin{array}{c}\text { Z.H. } \\
\text { V }\end{array}$ & $\begin{array}{l}\text { Mniš. } \\
\text { VI }\end{array}$ & $\begin{array}{l}\text { Švag. } \\
\text { VII }\end{array}$ & $\begin{array}{l}\text { Zám. } \\
\text { VIII }\end{array}$ & $\begin{array}{l}\text { Sob. } \\
\text { IX }\end{array}$ & $\begin{array}{l}\text { Mniš. } \\
\text { X }\end{array}$ & $\begin{array}{c}\text { Z.H. } \\
\text { XI }\end{array}$ \\
\hline $\mathrm{SiO}_{2}$ & 36.12 & 37.18 & 36.93 & 37.74 & 38.32 & 37.25 & 37.33 & 36.80 & 37.36 & 37.41 & 36.33 \\
\hline $\mathrm{TiO}_{2}$ & 2.25 & 1.39 & 1.47 & 1.36 & 2.01 & 1.54 & 1.63 & 3.35 & 2.29 & 1.76 & 2.38 \\
\hline $\mathrm{Al}_{2} \mathrm{O}_{3}$ & 14.37 & 16.44 & 15.72 & 17.11 & 15.34 & 15.22 & 17.40 & 15.06 & 15.46 & 18.82 & 17.09 \\
\hline $\mathrm{FeO}_{\text {anal }}$ & 19.43 & 15.55 & 16.46 & 16.81 & 14.06 & 16.40 & 14.53 & 19.11 & 16.07 & 16.17 & 18.31 \\
\hline $\mathrm{MnO}$ & 1.44 & 0.29 & 0.25 & 0.39 & 0.75 & 0.93 & 0.23 & n.d. & 0.12 & 0.36 & 0.26 \\
\hline $\mathrm{MgO}$ & 12.78 & 14.21 & 14.47 & 13.49 & 14.81 & 12.45 & 14.95 & 11.37 & 14.12 & 12.10 & 11.19 \\
\hline $\mathrm{CaO}$ & n.d. & n.d. & n.d. & 0.35 & 0.69 & n.d. & 0.43 & n.d. & 0.21 & n.d. & 0.02 \\
\hline $\mathrm{BaO}$ & n.a. & n.a. & n.a. & n.a. & n.a. & n.a. & n.a. & 2.11 & n.a. & n.a. & n.a. \\
\hline $\mathrm{Na}_{2} \mathrm{O}$ & 0.21 & 0.25 & 0.08 & n.d. & 0.07 & 0.11 & n.d. & n.d. & 0.10 & 0.08 & 0.12 \\
\hline $\mathrm{K}_{2} \mathrm{O}$ & 10.02 & 9.79 & 10.25 & 9.56 & 9.68 & 12.32 & 9.93 & 8.36 & 8.69 & 9.68 & 9.45 \\
\hline $\mathrm{FeO}_{\text {calc }}$ & 19.06 & 15.55 & 16.46 & 16.81 & 14.06 & 16.40 & 14.53 & 19.11 & 16.07 & 16.17 & 18.31 \\
\hline $\mathrm{Fe}_{2} \mathrm{O}_{3 \mathrm{calc}}$ & 0.42 & - & - & - & - & - & - & - & - & - & - \\
\hline $\mathrm{H}_{2} \mathrm{O}_{\text {calc }}$ & 3.91 & 3.94 & 3.76 & 3.57 & 3.26 & 3.45 & 3.57 & 3.36 & 3.79 & 3.25 & 3.87 \\
\hline total & 100.58 & 99.04 & 99.39 & 100.38 & 98.99 & 99.43 & 100.00 & 99.52 & 98.21 & 99.63 & 99.02 \\
\hline $\mathrm{Si}$ & 2.770 & 2.827 & 2.805 & 2.828 & 2.903 & 2.886 & 2.789 & 2.862 & 2.846 & 2.822 & 2.811 \\
\hline $\mathrm{Al}$ & 1.230 & 1.173 & 1.195 & 1.172 & 1.097 & 1.114 & 1.211 & 1.138 & 1.154 & 1.178 & 1.189 \\
\hline total & 4.000 & 4.000 & 4.000 & 4.000 & 4.000 & 4.000 & 4.000 & 4.000 & 4.000 & 4.000 & 4.000 \\
\hline $\mathrm{Ti}$ & 0.130 & 0.080 & 0.084 & 0.077 & 0.115 & 0.088 & 0.092 & 0.196 & 0.131 & 0.100 & 0.139 \\
\hline $\mathrm{Al}$ & 0.069 & 0.301 & 0.212 & 0.339 & 0.273 & 0.290 & 0.321 & 0.245 & 0.234 & 0.495 & 0.369 \\
\hline $\mathrm{Fe}^{3+}$ & 0.024 & - & - & - & - & - & - & - & - & - & - \\
\hline $\mathrm{Mg}$ & 1.461 & 1.611 & 1.638 & 1.506 & 1.673 & 1.465 & 1.665 & 1.318 & 1.603 & 1.361 & 1.291 \\
\hline $\mathrm{Fe}^{2+}$ & 1.222 & 0.989 & 1.045 & 1.053 & 0.891 & 1.074 & 0.908 & 1.243 & 1.024 & 1.021 & 1.184 \\
\hline $\mathrm{Mn}$ & 0.094 & 0.019 & 0.016 & 0.025 & 0.048 & 0.060 & 0.014 & - & 0.008 & 0.023 & 0.017 \\
\hline total & 3.000 & 3.000 & 3.000 & 3.000 & 3.000 & 3.000 & 3.000 & 3.000 & 3.000 & 3.000 & 3.000 \\
\hline $\mathrm{OH}$ & 2.000 & 1.902 & 1.904 & 1.786 & 1.651 & 1.759 & 1.782 & 1.741 & 1.895 & 1.639 & 1.542 \\
\hline $\mathrm{O}$ & - & 0.098 & 0.096 & 0.214 & 0.349 & 0.241 & 0.218 & 0.259 & 0.105 & 0.361 & 0.458 \\
\hline total & 2.000 & 2.000 & 2.000 & 2.000 & 2.000 & 2.000 & 2.000 & 2.000 & 2.000 & 2.000 & 2.000 \\
\hline $\mathrm{O}$ & 10.000 & 10.000 & 10.000 & 10.000 & 10.000 & 10.000 & 10.000 & 10.000 & 10.000 & 10.000 & 10.000 \\
\hline $\mathrm{Ca}$ & - & - & - & 0.028 & 0.056 & - & 0.035 & - & 0.017 & - & 0.002 \\
\hline $\mathrm{Ba}$ & - & - & - & - & - & - & - & 0.064 & - & - & - \\
\hline $\mathrm{Na}$ & 0.031 & 0.037 & 0.012 & - & 0.010 & 0.016 & - & - & 0.015 & 0.012 & 0.018 \\
\hline $\mathrm{K}$ & 0.980 & 0.950 & 0.993 & 0.914 & 0.936 & 1.007 & 0.946 & 0.830 & 0.845 & 0.932 & 0.933 \\
\hline total & 1.011 & 0.987 & 1.005 & 0.942 & 1.002 & 1.023 & 0.981 & 0.894 & 0.877 & 0.944 & 0.953 \\
\hline
\end{tabular}

Kyzový důl (IX and X), Zadní Hutisko (XI and XII) and Mnišské jámy (XIII);

3. hornblendites from Švagrov (XIV) or belonging to the Sobotín Massif (analyses from Fiala et al. 1980).

4. the host rock gneiss (samples are from Mnišské jámy; see Fig. 19).

Garnet occurs in garnetite, which is documented at four localities. Garnetite is the most abundant rock type in Zadní Hutisko (Figs 15d and 15e), relatively widely distributed in Švagrov (Fig. 12f), and rare at Kosaře and Mnišské jámy. In Kyzový důl, where garnetite sensu stricto was not detected, garnet crystals were found in quartz as relics only. All the garnet data are presented in Figs 20 and 21; selected analyses are summarized in Tab. 6 .
The magnetite analytical data are presented in Tab. 7, documenting that magnetite $\left(\mathrm{Fe}^{2+} \mathrm{Fe}^{3+}{ }_{2} \mathrm{O}_{4}\right)$ forms solid solutions with ulvite $\left(\mathrm{Fe}^{2+}{ }_{2} \mathrm{TiO}_{4}\right)$, jacobsite $\left(\mathrm{Mn}^{2+} \mathrm{Fe}^{3+}{ }_{2} \mathrm{O}_{4}\right)$, trevorite $\left(\mathrm{NiFe}^{3+}{ }_{2} \mathrm{O}_{4}\right)$, magnesioferrite $\left(\mathrm{MgFe}^{3+}{ }_{2} \mathrm{O}_{4}\right)$, hercynite $\left(\mathrm{Fe}^{2+} \mathrm{Al}_{2} \mathrm{O}_{4}\right)$ and chromite $\left(\mathrm{Fe}^{2+} \mathrm{Cr}_{2} \mathrm{O}_{4}\right)$ end members.

The analytical data for ilmenite obtained from magnetite-amphibole rocks (Tab. 8, I to III, V and VI), magnetite-chlorite-(biotite) rocks (VIII, IX, XI to XIV, XVI and XVII) and hornblendite (XIX) show that ilmenite $\left(\mathrm{Fe}^{2+} \mathrm{TiO}_{3}\right)$ forms solid solutions with the following end-members: pyrophanite $\left(\mathrm{Mn}^{2+} \mathrm{TiO}_{3}\right)$, geikielite $\left(\mathrm{MgTiO}_{3}\right)$, perovskite $\left(\mathrm{CaTiO}_{3}\right)$, hematite $\left(\mathrm{Fe}^{3+}{ }_{2} \mathrm{O}_{3}\right)$, and eskolaite $\left(\mathrm{Cr}_{2} \mathrm{O}_{3}\right)$. The ilmenite solid 
Tab. 5 Selected chlorite analyses of magnetite-amphibole rocks from Rudná hora (I and II), Kosaře (III and IV) and Švagrov (V and VI); magnetite-chlorite-(biotite) rocks of Břidličná (VII and VIII), Kyzový důl (IX and X), Zadní Hutisko (XI and XII) and Mnišské jámy (Mniš.) (XIII); hornblendite of Švagrov (Švagr.) (XIV); and Lahn-Dill type samples of the Malý děd Mt. (XV and XVI). In wt. \% (upper part) and apfu (lower part), including the end-members $\mathrm{Mg}, \mathrm{Fe}_{\text {tot }}$ and $\mathrm{Al}^{\mathrm{VI}}$ (in mol. \%).

\begin{tabular}{|c|c|c|c|c|c|c|c|c|c|c|c|c|c|c|c|c|}
\hline & \multicolumn{6}{|c|}{ Magnetite-amphibole rocks } & \multicolumn{7}{|c|}{ Magnetite-chlorite-(biotite) rocks } & \multirow{3}{*}{$\frac{\frac{\text { Hornbl. }}{\text { Švagr. }}}{\text { XIV }}$} & \multirow{2}{*}{\multicolumn{2}{|c|}{$\frac{\text { Lahn-Dill type }}{\text { Malý Dĕd Mt. }}$}} \\
\hline & \multicolumn{2}{|c|}{ Rudná hora } & \multicolumn{2}{|c|}{ Kosaře } & \multicolumn{2}{|c|}{ Švagrov } & \multicolumn{2}{|c|}{ Břidličná } & \multicolumn{2}{|c|}{ Kyzový důl } & \multicolumn{2}{|c|}{ Zad. Hutisko } & \multirow{2}{*}{$\frac{\text { Mniš. }}{\text { XIII }}$} & & & \\
\hline & I & II & III & IV & V & VI & VII & VIII & IX & $\mathbf{X}$ & XI & XII & & & XV & XVI \\
\hline $\mathrm{SiO}_{2}$ & 23.86 & 25.19 & 24.99 & 26.29 & 26.75 & 24.99 & 27.34 & & 25.78 & 26.78 & 27.67 & 28.87 & 26.80 & 22.03 & 22.28 & 22.76 \\
\hline $\mathrm{TiO}_{2}$ & 0.12 & 0.07 & n.d. & n.d. & n.d. & n.d. & 0.07 & 0.11 & 0.05 & n.d. & n.d. & n.d. & 0.07 & 0.14 & 0.06 & 0.06 \\
\hline $\mathrm{Al}_{2} \mathrm{O}_{3}$ & 23.79 & 24.69 & 22.45 & 20.51 & 20.25 & 22.45 & 21.26 & 21.18 & 18.57 & 20.20 & 20.24 & 17.21 & 21.49 & 25.68 & 21.45 & 21.10 \\
\hline $\mathrm{FeO}_{\text {anal }}$ & 19.30 & 18.44 & 17.87 & 15.17 & 21.24 & 17.87 & 17.98 & 18.62 & 30.39 & 19.61 & 18.31 & 20.88 & 18.80 & 17.26 & 40.71 & 40.91 \\
\hline $\mathrm{MnO}$ & 50 & 0.45 & 0.39 & 0.32 & 0.41 & 0.39 & 0.25 & 0.37 & 0.45 & 0.35 & 1.91 & 2.31 & 0.38 & 0.19 & 0.04 & 0.03 \\
\hline $\mathrm{MgO}$ & 20.86 & 19.02 & 21.31 & 23.58 & 18.82 & 21.31 & 20.32 & 20.32 & 14.74 & 21.49 & & 19.31 & 20.28 & 22.24 & 4.11 & 4.15 \\
\hline $\mathrm{CaO}$ & n.d. & 0.08 & 0.58 & 1.99 & n.d. & 0.58 & n.d. & n.d. & n.d. & 0.24 & 0.41 & n.d. & n.d. & 0.11 & 0.02 & 0.04 \\
\hline $\mathrm{FeO}_{\text {calc }}$ & 15.03 & 18.44 & 14.46 & 10.63 & 20.94 & 14.34 & 17.98 & 18.62 & 26.97 & 16.84 & 18.31 & 20.88 & 18.80 & 10.48 & 40.29 & 40.88 \\
\hline $\mathrm{Fe}_{2} \mathrm{O}_{3 \text { calc }}$ & 4.74 & - & 3.79 & 5.04 & 0.33 & 3.93 & - & - & 3.80 & 3.08 & - & - & - & 7.53 & 47 & 0.03 \\
\hline $\mathrm{H}_{2} \mathrm{O}_{\text {calc }}$ & 11.91 & 11.72 & 11.87 & 12.07 & 11.63 & 11.90 & 11.52 & 11.71 & 11.44 & 11.93 & 10.02 & 11.67 & 11.76 & 12.01 & 10.53 & 10.57 \\
\hline total & 100.63 & 99.66 & 99.84 & 100.43 & 99.13 & 99.89 & 98.74 & 98.85 & 101.80 & 100.91 & 99.49 & 100.25 & 99.58 & 100.41 & 99.25 & 99.62 \\
\hline $\mathrm{Si}$ & 401 & 2.554 & 2.521 & 2.611 & 2.757 & & 2.790 & & & 2.688 & & & 2.723 & 2.197 & & 2.582 \\
\hline $\mathrm{Al}$ & .599 & 1.446 & 1.479 & 1.389 & 1.243 & & 1.210 & 1.285 & 1.300 & 1.312 & & 1.035 & 1.277 & 1.803 & & 1.418 \\
\hline total & 4.000 & 4.000 & 4.000 & 4.000 & 4.000 & 4.000 & 4.000 & 4.000 & 4.000 & 4.000 & 4.000 & 8.000 & 4.000 & 4.000 & 4.000 & 4.000 \\
\hline $\mathrm{Ti}$ & 0.009 & 0.005 & - & - & - & - & 0.006 & 0.009 & 0.004 & - & - & - & 0.005 & 0.011 & 0.005 & 0.006 \\
\hline $\mathrm{Al}$ & .222 & 1.505 & 1.191 & 1.012 & 1.217 & 1.181 & 1.347 & 1.268 & 0.922 & 1.079 & 1.642 & 1.049 & 1.295 & 1.216 & 1.414 & 1.403 \\
\hline $\mathrm{Fe}^{3+}$ & 0.359 & - & 0.288 & 0.377 & 0.026 & 0.302 & - & - & 0.300 & 0.233 & - & - & - & .565 & 0.040 & 0.003 \\
\hline Mg & 103 & 2.879 & 3.205 & 3.490 & 2.891 & & 3.091 & 3.098 & 2.301 & 3.217 & 2.456 & 2.956 & 3.070 & 3.306 & 0.698 & 0.702 \\
\hline $\mathrm{Fe}^{2+}$ & 1.265 & 1.564 & 1.220 & 0.883 & 1.831 & 1.224 & 1.535 & 1.593 & 2.363 & 1.414 & 1.677 & 1.794 & 1.597 & 0.874 & 3.836 & 3.878 \\
\hline $\mathrm{Mn}$ & 0.042 & 0.038 & 0.033 & 0.027 & 0.035 & 0.033 & 0.021 & 0.032 & 0.040 & 00.030 & 0.177 & 0.402 & 0.033 & 0.016 & 0.004 & 0.003 \\
\hline $\mathrm{Ca}$ & - & 0.009 & 0.063 & 0.211 & - & 0.062 & - & - & - & 0.027 & 0.048 & - & - & 0.012 & 0.003 & 0.005 \\
\hline total & 6.000 & 6.000 & 6.000 & 6.000 & 6.000 & & 6.000 & 6.000 & 6.000 & 6.000 & 6.000 & 6.000 & 6.000 & 6.000 & 6.000 & 6.000 \\
\hline $\mathrm{OH}$ & 8.000 & 7.936 & 8.000 & 8.000 & 8.000 & 8.000 & 7.851 & 8.000 & 8.000 & 8.000 & 7.328 & 7.986 & 7.972 & 8.000 & 8.000 & 8.000 \\
\hline $\mathrm{O}$ & - & 0.064 & - & - & - & - & 0.149 & - & - & - & 0.672 & 0.014 & 0.028 & - & - & - \\
\hline total & 8.000 & 8.000 & 8.000 & 8.000 & 8.000 & 8.000 & 8.000 & 8.000 & 8.000 & 8.000 & 8.000 & 8.000 & 8.000 & 8.000 & 8.000 & 8.000 \\
\hline O & 10.000 & 10.000 & 10.000 & 10.000 & 10.000 & 10.000 & 10.000 & 10.000 & 10.000 & 10.000 & 10.000 & 10.000 & 10.000 & 10.000 & 10.000 & 10.000 \\
\hline $\mathrm{Mg}$ & 52.16 & 48.21 & 54.29 & 60.57 & 48.47 & & 51.75 & 51.99 & 38.63 & 54.13 & 42.53 & 50.97 & 51.49 & 55.46 & 11.66 & 11.73 \\
\hline $\mathrm{Fe}_{\text {tot }}$ & 27.30 & 26.24 & 25.54 & 21.87 & 31.13 & 25.84 & 25.70 & 26.73 & 44.71 & 27.71 & 29.04 & 30.94 & 26.79 & 24.14 & 64.73 & 64.83 \\
\hline $\mathrm{Al}^{\mathrm{VI}}$ & 20.54 & 25.25 & 20.17 & 17.56 & 20.40 & 20.00 & 22.55 & 21.28 & 16.66 & 18.16 & 28.43 & 18.09 & 21.72 & 20.40 & 23.61 & 23.44 \\
\hline
\end{tabular}

\section{$\mathrm{Mg}$}

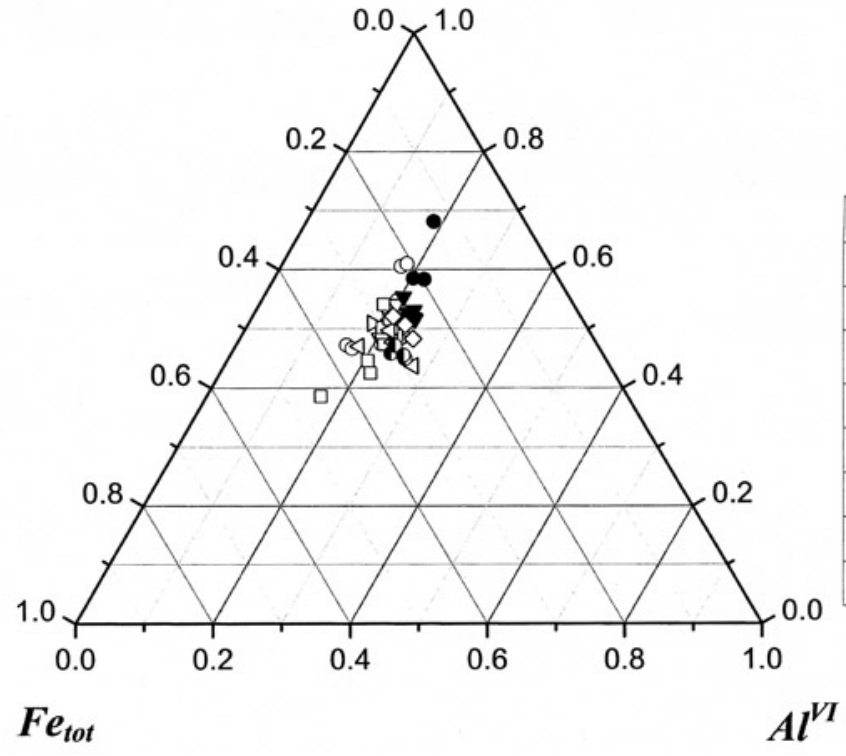

\begin{tabular}{|c|c|c|}
\hline & Sobotin Massif & • \\
\hline$\diamond$ & Rudná hora & \\
\hline 0 & Kosaře & \\
\hline$\nabla$ & Švagrov & $\boldsymbol{\nabla}$ \\
\hline$D$ & Zadní Hutisko & \\
\hline 占 & Bř́idličná & \\
\hline$\square$ & $K y z o v \dot{y} d \dot{u} l$ & \\
\hline$\triangleleft$ & Mnišské jámy & \\
\hline Gneiss & Desná Group & D \\
\hline
\end{tabular}

Fig. 18 Chlorite compositions in mol. \% presented in the ternary diagram $\mathrm{Fe}_{\text {tot }}-\mathrm{Mg}-\mathrm{Al}^{\mathrm{VI}}$ according to Mücke and Farshad (2005). 
Tab. 6 Selected garnet analyses for garnetite from Kosaře (I to III= average of 9 analyses), Švagrov (IV to VI = average of 7 analyses), Kyzový důl (VII to IX = average of 6 analyses), Zadní Hutisko (X to XII = average of 12 analyses), and Mnišské jámy (XIII to XV = average of 8 analyses). In wt. \% (upper part) and end-member compositions (in mol. \%; lower part). The data of each locality represent analyses with highest, lowest and averaged $\mathrm{Mn}$ and Fe contents.

\begin{tabular}{|c|c|c|c|c|c|c|c|c|c|c|c|c|c|c|c|}
\hline & \multicolumn{3}{|c|}{ Kosaře } & \multicolumn{3}{|c|}{ Švagrov } & \multicolumn{3}{|c|}{ Kyzový důl } & \multicolumn{3}{|c|}{ Zadní Hutisko } & \multicolumn{3}{|c|}{ Mnišské jámy } \\
\hline & I & II & III, 9 & IV & V & VI, 7 & VII & VIII & IX, 6 & $\mathrm{X}$ & XI & XII,12 & XIII & XIV & $\mathrm{XV}, 8$ \\
\hline $\mathrm{SiO}_{2}$ & 37.07 & 36.82 & 36.90 & 36.80 & 37.27 & 37.31 & 36.98 & 37.32 & 37.67 & 37.05 & 36.70 & 36.88 & 37.10 & 36.91 & 37.26 \\
\hline $\mathrm{TiO}_{2}$ & 0.03 & 0.08 & 0.12 & 0.13 & n.d. & 0.14 & n.d. & n.d. & n.d. & n.d. & n.d. & n.d. & 0.02 & 0.13 & 0.08 \\
\hline $\mathrm{Al}_{2} \mathrm{O}_{3}$ & 20.06 & 21.17 & 19.95 & 18.75 & 19.33 & 19.39 & 19.57 & 19.11 & 19.44 & 19.08 & 20.52 & 19.32 & 20.54 & 19.94 & 20.21 \\
\hline $\mathrm{FeO}_{\text {anal }}$ & 23.36 & 19.39 & 22.13 & 25.96 & 19.88 & 21.09 & 16.85 & 15.46 & 15.54 & 11.14 & 18.61 & 14.27 & 22.72 & 19.86 & 21.16 \\
\hline $\mathrm{MnO}$ & 13.32 & 12.91 & 13.48 & 11.35 & 14.86 & 14.89 & 16.66 & 17.33 & 17.26 & 28.14 & 19.79 & 22.86 & 11.33 & 13.89 & 12.75 \\
\hline $\mathrm{MgO}$ & 2.49 & 2.35 & 2.48 & 3.04 & 2.30 & 2.48 & 3.10 & 2.89 & 2.86 & 1.82 & 1.53 & 1.70 & 1.55 & 0.89 & 1.29 \\
\hline $\mathrm{CaO}$ & 3.42 & 7.23 & 4.69 & 4.52 & 6.63 & 4.92 & 7.43 & 8.01 & 7.45 & 3.64 & 2.25 & 4.88 & 7.20 & 8.50 & 7.59 \\
\hline $\mathrm{FeO}_{\text {calc }}$ & 22.06 & 19.27 & 20.62 & 22.64 & 17.29 & 18.69 & 14.29 & 12.40 & 12.91 & 8.34 & 18.26 & 12.00 & 21.79 & 18.27 & 19.85 \\
\hline $\mathrm{Fe}_{2} \mathrm{O}_{3 \text { calc }}$ & 1.45 & 0.13 & 1.68 & 3.69 & 2.88 & 2.67 & 2.85 & 3.40 & 2.92 & 3.16 & 0.39 & 2.52 & 1.03 & 1.77 & 1.46 \\
\hline total & 99.90 & 99.96 & 99.92 & 100.42 & 100.56 & 100.49 & 100.88 & 100.46 & 100.51 & 101.23 & 99.44 & 100.16 & 100.56 & 100.30 & 100.49 \\
\hline Almandite & 49.73 & 42.08 & 45.97 & 49.85 & 38.45 & 42.14 & 30.92 & 27.35 & 28.67 & 18.65 & 41.58 & 27.10 & 48.14 & 40.76 & 44.23 \\
\hline Spessartite & 30.40 & 28.56 & 30.44 & 25.37 & 33.47 & 33.83 & 36.52 & 38.69 & 38.85 & 63.70 & 45.65 & 51.94 & 25.35 & 31.40 & 28.90 \\
\hline Grossular & 5.47 & 19.81 & 8.67 & 1.58 & 10.26 & 6.08 & 12.30 & 12.50 & 12.43 & 1.00 & 5.37 & 6.43 & 17.30 & 18.95 & 17.32 \\
\hline Andradite & 4.33 & 0.25 & 4.73 & 10.96 & 8.66 & 7.75 & 8.32 & 10.12 & 8.75 & 9.41 & 1.20 & 7.70 & 3.07 & 5.07 & 4.25 \\
\hline Pyrope & 10.00 & 9.13 & 9.85 & 11.94 & 9.16 & 9.90 & 11.94 & 11.34 & 11.30 & 7.24 & 6.20 & 6.83 & 6.11 & 3.55 & 5.13 \\
\hline Schorlomite & 0.07 & 0.17 & 0.34 & 0.30 & - & 0.30 & - & - & - & - & - & - & 0.03 & - & 0.17 \\
\hline
\end{tabular}

\begin{tabular}{|c|c|c|}
\hline & Sobotín Massif & $\bullet$ \\
\hline$\diamond$ & Rudná hora & \\
\hline 0 & Kosaře & \\
\hline$\nabla$ & Švagrov & $\boldsymbol{\nabla}$ \\
\hline$\triangleright$ & Zadni Hutisko & \\
\hline t & B řidličná & \\
\hline$\square$ & Kyzovýd ål & \\
\hline$\triangleleft$ & Mnišské jámy & \\
\hline Gneiss & Desná Group & D \\
\hline
\end{tabular}

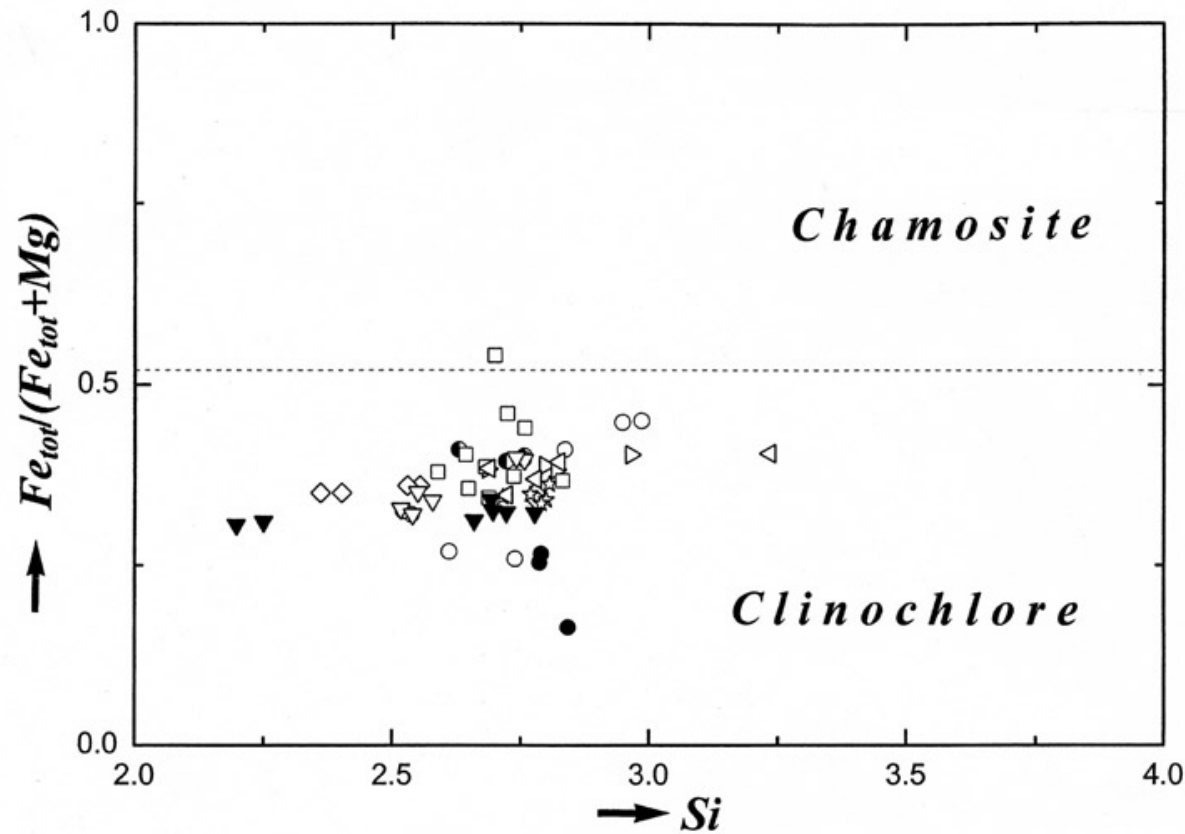

Fig. 19 Chlorite diagram in at. \% after Melka (1965; strongly modified, as many chlorite end members have been abolished).

solutions occur in the form of (1) oriented intergrowths with magnetite (Fig. 22a), (2) as elongated crystals or aggregates among magnetite and/or amphibole crystals (Fig. 22b), which may be partially replaced by titanite, as well as crystals that contain exsolutions of (4) hematite (Fig. 22c), (5) corundum (Fig. 22d) or (6) relics within newly-formed blitz-textured intergrowth of hematite and rutile.
Apatite appears in varying proportions in all rock types, including hornblendite of both the Sobotín Massif (Rudná hora) and Švagrov (Fig. 13e), magnetite-amphibole rocks of Kosaře (Fig. 12e) and Švagrov (Fig. 13c) and magnetite-chlorite-(biotite)-rocks. Generally, apatite forms rounded to euhedral grains (up to $0.2 \mathrm{~mm}$ ) and is mainly evenly distributed. The whole-rock $\mathrm{P}_{2} \mathrm{O}_{5}$ concentrations fall in the range of 0.1 to $0.7 \mathrm{wt} . \%$ 
Tab. 7 Selected magnetite analyses in wt. \% (upper part) and the proportions of the end-members in mol. \% (lower part). I: Sylvani (average of 10 analyses), II: Sylvani (highest Mn), III: Rudná hora (R. h.); IV: Zadní Hutisko (highest $\mathrm{Al}_{2} \mathrm{O}_{3}$ ), V: Zadní Hutisko (highest Mn), VI: Kosaře (Kos.), VII: Kyzový důl (average of 5 analyses), VIII: Kyzový důl (highest $\mathrm{Al}_{2} \mathrm{O}_{3}$ ), IX: Švagrov (highest $\mathrm{TiO}_{2}$ and $\mathrm{Al}_{2} \mathrm{O}_{3}$ contents), X: Švagrov (highest $\mathrm{Cr}_{2} \mathrm{O}_{3}$ ), XI: Mnišské jámy (average of 8 analyses) and XII: Mnišské jámy (highest $\mathrm{Cr}_{2} \mathrm{O}_{3}$ and $\mathrm{TiO}_{2}$ ).

\begin{tabular}{|c|c|c|c|c|c|c|c|c|c|c|c|c|}
\hline & \multicolumn{2}{|c|}{ Sylvani } & \multirow{2}{*}{$\begin{array}{c}\text { R. h. } \\
\text { III }\end{array}$} & \multicolumn{2}{|c|}{ Zadní Hutisko } & \multirow{2}{*}{$\begin{array}{c}\text { Kos. } \\
\text { VI }\end{array}$} & \multicolumn{2}{|c|}{ Kyzový důl } & \multicolumn{2}{|c|}{ Švagrov } & \multicolumn{2}{|c|}{ Mnišské jámy } \\
\hline & $I_{10}$ & II & & IV & $\mathbf{V}$ & & $\mathrm{VII}_{5}$ & VIII & IX & $\mathrm{X}$ & $\mathrm{XI}_{8}$ & XII \\
\hline $\mathrm{FeO}$ & 92.73 & 92.07 & 92.16 & 92.34 & 93.47 & 91.99 & 93.38 & 92.64 & 93.07 & 92.87 & 93.39 & 93.03 \\
\hline $\mathrm{TiO}_{2}$ & 0.12 & 0.12 & 0.15 & 0.04 & 0.08 & 0.12 & 0.30 & 0.04 & 0.09 & 0.11 & 0.14 & 0.89 \\
\hline $\mathrm{MnO}$ & 0.21 & 0.69 & 0.18 & 0.07 & 0.25 & 0.05 & 0.07 & 0.07 & 0.16 & 0.12 & 0.14 & 0.22 \\
\hline $\mathrm{NiO}$ & 0.06 & n.d. & 0.01 & 0.14 & n.d. & n.d. & 0.03 & n.a. & n.a. & n.a. & n.d. & 0.05 \\
\hline $\mathrm{MgO}$ & 0.07 & n.d. & n.d. & 0.02 & n.d. & 0.01 & 0.02 & 0.06 & n.d. & n.d. & n.d. & n.d \\
\hline $\mathrm{Cr}_{2} \mathrm{O}_{3}$ & 0.02 & n.d. & 0.01 & 0.05 & n.d. & 0.03 & 0.05 & n.a. & n.a. & 0.03 & 0.01 & 0.09 \\
\hline $\mathrm{Al}_{2} \mathrm{O}_{3}$ & 0.16 & 0.04 & 0.29 & 0.14 & 0.10 & 0.08 & 0.05 & 0.15 & 0.23 & 0.10 & 0.08 & 0.02 \\
\hline $\mathrm{FeO}_{\text {calc }}$ & 30.89 & 30.42 & 30.91 & 30.79 & 31.16 & 30.96 & 31.45 & 30.89 & 31.15 & 31.09 & 31.24 & 31.91 \\
\hline $\mathrm{Fe}_{2} \mathrm{O}_{3 \text { calc }}$ & 68.73 & 68.52 & 68.07 & 68.41 & 69.25 & 67.83 & 68.83 & 68.63 & 68.82 & 68.66 & 69.07 & 67.93 \\
\hline total & 100.26 & 99.79 & 99.61 & 99.66 & 100.54 & 99.08 & 100.80 & 99.84 & 100.45 & 100.11 & 100.68 & 101.11 \\
\hline $\mathrm{FeFe}_{2} \mathrm{O}_{4}$ & 98.00 & 97.40 & 98.35 & 98.80 & 98.75 & 99.25 & 98.50 & 99.05 & 98.70 & 99.00 & 98.90 & 96.40 \\
\hline $\mathrm{Fe}_{2} \mathrm{TiO}_{4}$ & 0.30 & 0.30 & 0.40 & 0.10 & 0.20 & 0.30 & 0.90 & 0.10 & 0.30 & 0.30 & 0.40 & 2.50 \\
\hline $\mathrm{MnFe}_{2} \mathrm{O}_{4}$ & 0.70 & 2.20 & 0.60 & 0.20 & 0.80 & 0.20 & 0.20 & 0.20 & 0.50 & 0.40 & 0.50 & 0.70 \\
\hline $\mathrm{NiFe}_{2} \mathrm{O}_{4}$ & 0.20 & - & - & 0.40 & - & - & 0.10 & - & - & - & - & 0.20 \\
\hline $\mathrm{MgFe}_{2} \mathrm{O}_{4}$ & 0.40 & - & - & 0.10 & - & - & 0.10 & 0.30 & - & - & - & - \\
\hline $\mathrm{FeAl}_{2} \mathrm{O}_{4}$ & 0.35 & 0.10 & 0.65 & 0.30 & 0.25 & 0.20 & 0.10 & 0.35 & 0.50 & 0.25 & 0.20 & 0.05 \\
\hline $\mathrm{FeCr}_{2} \mathrm{O}_{4}$ & 0.05 & - & - & 0.10 & - & 0.05 & 0.10 & - & - & 0.05 & - & 0.15 \\
\hline
\end{tabular}

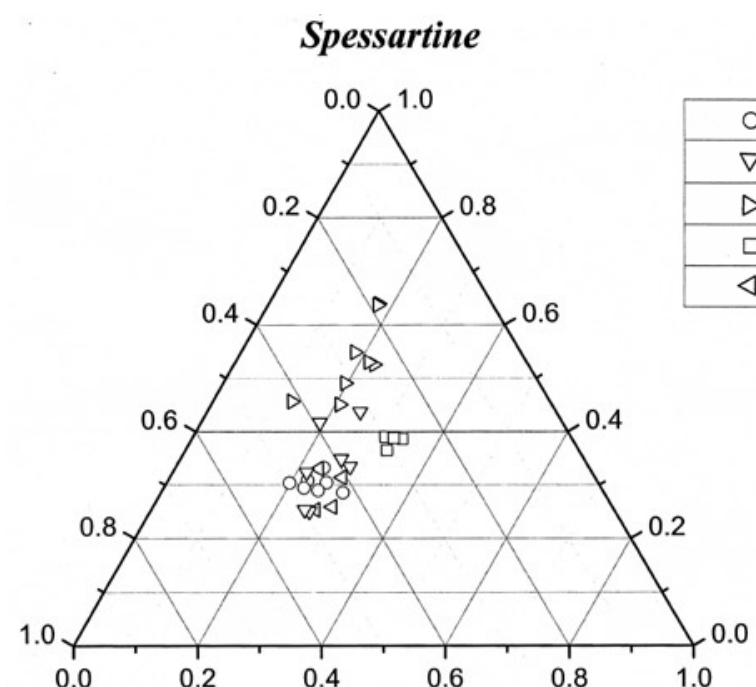

Almandine

Fig. 20 Garnet compositions in mol. \% presented in the ternary diagram Almandine - Spessartine - "Other end-members" (grossular, andradite, and pyrope - see Fig. 21).

(on average $0.5 \mathrm{wt} . \%$ ). The highest content of the apatite is in magnetite-amphibole rocks (Fig. 13c) and hornblendite (Fig. 13e) of Švagrov, containing $5.47 \mathrm{wt}$. $\% \mathrm{P}_{2} \mathrm{O}_{5}$ (Tab. 11, I), corresponding to about 20 vol. $\%$ of apatite. As proven by qualitative analyses, carried out in all locations, apatite contains always $\mathrm{F}$, whereas $\mathrm{Cl}$ was never detected. Selected quantitative analyses were car-

\section{Andradite}

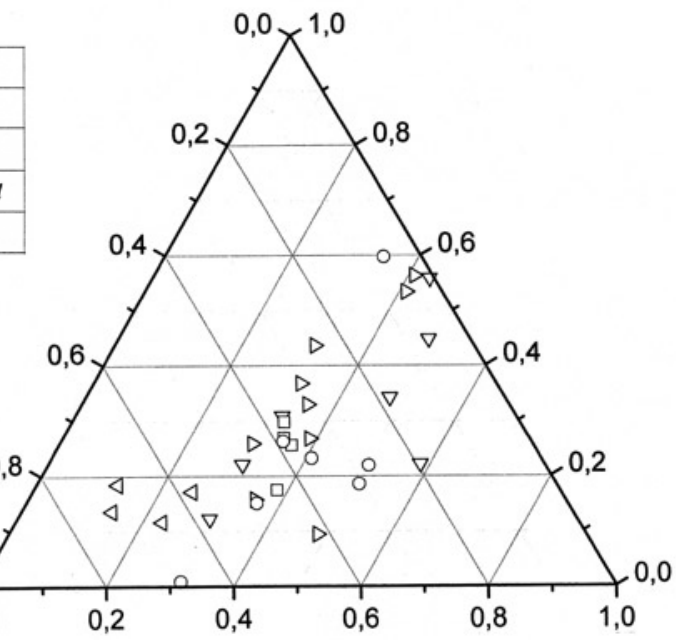

Pyrope

Fig. 21 Proportions of the minor components in garnets (Grossular - Andradite - Pyrope diagram).

ried out but are not published. They show that the amount of $\mathrm{F}$ is mostly close to $3.2 \mathrm{wt}$. \%. For comparison, the theoretical pure F-apatite $\mathrm{Ca}_{5}\left[\mathrm{~F} /\left(\mathrm{PO}_{4}\right)_{3}\right]$ contains 3.77 wt. \% F. Consequently, the studied apatite is $(\mathrm{OH})$-bearing F-apatite. Apart from $\mathrm{CaO}$ and $\mathrm{P}_{2} \mathrm{O}_{5}$, other elements contained in apatite are $\mathrm{Fe}(0.16$ to $0.70 \mathrm{wt} . \% \mathrm{FeO})$ and Mn (0.04 to 0.48 wt. \% MnO). 
Feldspar was analysed in a few samples, being the most abundant in magnetite-chlorite(biotite) rocks and in paragenesis with chlorite, carbonate and epidote. In magnetite-rich rocks of Kyzový důl, feldspar represents up to 5 vol. \% (Fig. 14a). In magnetite-amphibole rocks this mineral occurs especially in samples with amphibole replaced by epidote. The feldspar is mainly albite $\left(\mathrm{An}_{12.79-0.73}\right.$ mol. \%) (Břidličná, Zadní Hutisko and Mnišské jámy: Tab. 9, III to VII). The highest but still low anorthite contents were obtained in magnetite-chlorite-(biotite) rocks of Kyzový důl ( $\mathrm{An}_{12.79}$ mol. \%; II). Anorthite-rich plagioclase was detected in metahornblendite of Švagrov $\left(\mathrm{An}_{50.21-51.45} \mathrm{~mol} . \%\right.$; VI $)$ and Zadní Hutisko $\left(\mathrm{An}_{23,6}\right.$ mol. \%; VI). Feldspar from Kosaře, found as inclusion in amphibole, is pure K-feldspar (Tab. 9; I). Albite was also detected in Kosaře.

Carbonate is similar in its distribution to feldspar. Relatively rich in carbonate are the magnetite-chlorite-(biotite) rocks at Břidličná, Mnišské jámy and in particular in Kyzový důl (Figs 14c and 14d). In Rudná hora, Sylvani and Švagrov, the carbonate occurs in magnetite-amphibole rocks and in Zadní Hutisko in albitized metahornblendite. Carbonates are always associated with albite, often forming inclusions in this mineral or the two being intimately intergrown. Carbonate is composed predominantly of the $\mathrm{CaCO}_{3}$ end-member, varying from 88.85 (Kyzový důl) to $99.22 \mathrm{~mol}$. \% (Zadní Hutisko) followed by $\mathrm{MnCO}_{3}(0.04$ to 7.47 mol. \%), $\mathrm{FeCO}_{3}(0.49$ to $3.77 \mathrm{~mol} . \%)$ and $\mathrm{MgCO}_{3}(0.00$ to $3.88 \mathrm{~mol}$. \%) (Tab. 10). The carbonate solid solutions are usually relatively constant in composition. Only in Kyzový důl occur three carbonate types. Two of them belong to the calcite series with $88.9 \mathrm{~mol}$. \% $\mathrm{CaCO}_{3}$ accompanied by $\mathrm{FeCO}_{3}, \mathrm{MnCO}_{3}$ and $\mathrm{MgCO}_{3}$ in nearly equal proportions (higher than 3 mol. \%); and $\mathrm{CaCO}_{3}$ with high $\mathrm{MnCO}_{3}$ proportion $(7.5 \mathrm{~mol}$. \%). The third type is represented by kutnohorite-bearing $(5.8 \mathrm{~mol} . \%)$ and ankerite-rich $(24.0 \mathrm{~mol}$. \%) dolomite (Tab. 10).

Epidote occurs in magnetite-amphibole rocks and hornblendites, where it replaced predominantly amphibole. This replacement may be strongly developed in Švagrov, Kosaře and Zadní Hutisko (Fig. 15f), where rocks may contain abundant epidote pseudomorphs after amphibole (Fig. 12d). In magnetite-chlorite-(biotite) rocks, epidote is less abundant. 

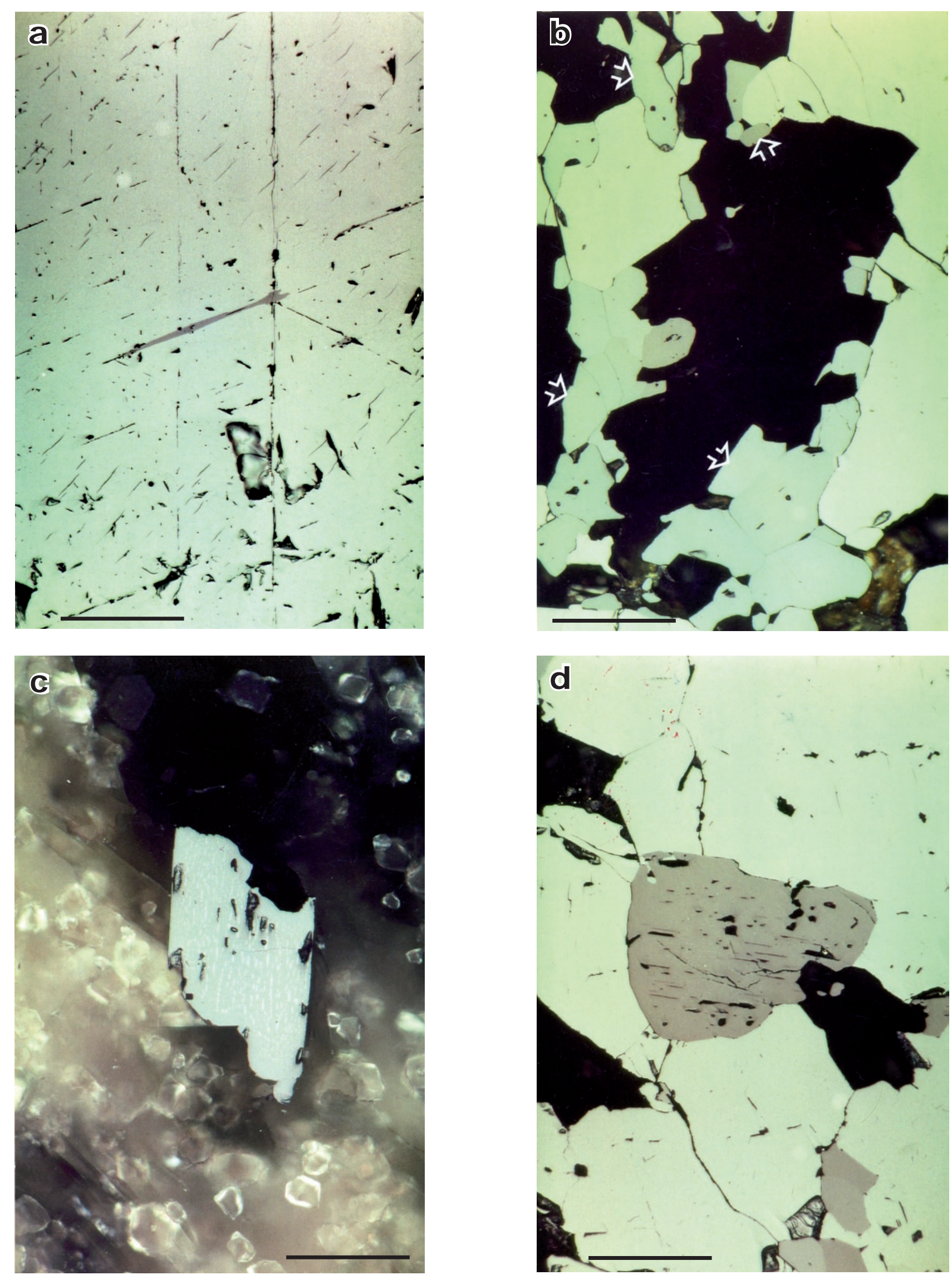
Tab. 9 Selected feldspar electron-microprobe analyses of Kosaře (I), Kyzový důl (II), Břidličná (III), Zadní Hutisko (IV to VI), Mnišské jámy (VII), and Švagrov (VIII) presented in the form of end-members (mol. \%).

\begin{tabular}{|c|c|c|c|c|c|c|c|c|}
\hline & Kosaře & Kyzový důl & Břidl. & & Iní Huti & & Mnišské jámy & Švagrov \\
\hline & I & II & III & IV & $\mathbf{V}$ & VI & VII & VIII \\
\hline Albite & - & $86.80-97.71$ & 98.96 & 97.71 & 96.01 & 76.02 & 98.35 & $48.13-49.38$ \\
\hline Anorthite & 0.003 & $12.79-1.98$ & 0.73 & 1.98 & 3.80 & 23.58 & 0.97 & $51.45-50.21$ \\
\hline K-feldspar & 0.997 & $0.41-0.31$ & 0.31 & 0.31 & 0.19 & 0.40 & 0.68 & $0.42-0.41$ \\
\hline
\end{tabular}

Tab. 10 Selected carbonate electron-microprobe analyses from Rudná hora, Sylvani, Švagrov, Břidličná, Mnišské jámy, Kyzový důl (three types), Zadní Hutisko and Malý Dĕd presented in the form of end-members of the calcite and the dolomite series (mol. \%).

\begin{tabular}{|c|c|c|c|c|c|}
\hline & $\mathbf{\Sigma N}$ & $\mathrm{CaCO}_{3}$ & $\mathrm{FeCO}_{3}$ & $\mathrm{MnCO}_{3}$ & $\mathrm{MgCO}_{3}$ \\
\hline Rudná hora & 4 & 89.24 & 3.77 & 3.11 & 3.88 \\
\hline Sylvani & 4 & 99.13 & 0.49 & 0.38 & - \\
\hline Švagrov & 4 & 95.19 & 1.77 & 1.81 & 1.23 \\
\hline Břidličná & 3 & 92.52 & 2.78 & 2.06 & 2.64 \\
\hline Mnišské jámy & 3 & 94.16 & 2.02 & 1.91 & 1.91 \\
\hline \multirow{2}{*}{ Kyzový důl } & 10 & 88.85 & 3.47 & 3.05 & 3.63 \\
\hline & 4 & 91.02 & 1.51 & 7.47 & - \\
\hline \multirow[t]{2}{*}{ Zadní Hutisko } & 3 & 99.22 & 0.74 & 0.04 & - \\
\hline & & $\mathrm{CaCO}_{3}$ & $\mathrm{CaFe}\left(\mathrm{CO}_{3}\right)_{2}$ & $\mathrm{CaMn}\left(\mathrm{CO}_{3}\right)_{2}$ & $\mathrm{CaMg}\left(\mathrm{CO}_{3}\right)_{2}$ \\
\hline Kyzový důl & 2 & 3.38 & 24.00 & 5.82 & 66.80 \\
\hline Malý Děd Mt. & 5 & 0.24 & 84.56 & 0.42 & 14.36 \\
\hline
\end{tabular}

\section{Discussion}

\subsection{Whole-rock analyses}

The whole-rock compositions can be briefly summarized as follows: Tabs 11 and 12 contain analytical data from hornblendites (Tab. 11, I to VI) and magnetite-amphibole rocks (Tab. 11, VII to X, and Tab. 12, IX to XIII) [gneisses (Tab. 11, XI to XIII) were analyzed for completeness only]. All these data are presented in the $\left(\mathrm{Na}_{2} \mathrm{O}\right.$ $\left.+\mathrm{K}_{2} \mathrm{O}\right)-\mathrm{MgO}-\mathrm{FeO}_{\text {tot }}$ diagram (Fig. 23). The data show a clear separation of the magnetite-amphibole rocks from those that represent hornblendites. Magnetite-amphibole rocks occupy a small field close to the $\mathrm{FeO}_{\text {tot }}$ apex. The hornblendites are concentrated within a larger field, and this confirms that they are similar in composition. However, two points for hornblendite of the Sobotín Massif and hornblendite from Švagrov located within the Desná

Fig. 22a - Magnetite intergrown with spinel lamellae (black) parallel to (100) and a bigger ilmenite lamella parallel to (111) (in the centre). Reflected light, oil immersion, scale bar $5 \mu \mathrm{m}$, Sylvani; $\mathbf{b}$ - Grain boundaries of magnetite (bright) and amphibole (black) are occupied by ilmenite (bireflecting; arrows). Reflected light, oil immersion, scale bar $5 \mu \mathrm{m}$, Sylvani; c - Ilmenite containing hematite exsolutions. Rounded crystals with internal reflections are garnets, which are embedded in quartz. Reflected light, oil immersion, scale bar $5 \mu \mathrm{m}$, Švagrov; d - Inclusion of ilmenite containing oriented lamellae of corundum (black, small needles) in magnetite, which is associated with amphibole (dark). Reflected light, oil immersion, scale bar $5 \mu \mathrm{m}$, Sylvani. gneisses, are nearly identical (see also Tab. 11, IV and V). One point falling between the two fields represents the Sylvani rock sample, which is shown in Fig. 2. This sample contains both, hornblendite as well as magnetite-amphibole rock.

Table 13 contains analytical data from magnetite-rich samples enriched in laboratory, with magnetite originally occurring in paragenesis with amphibole (I to III and IX) or chlorite-(biotite) (IV to VII and X). The remaining analyses are from compact magnetite, which was concentrated by natural processes (VIII, XI and XII). There are no obvious differences in the major-element and traceelement concentrations between the two rock groups (e.g., $\mathrm{Zn}, \mathrm{Ni}, \mathrm{Co}, \mathrm{Cr}$ and V). Table 13 also reveals that magnetite is accompanied by relatively high $\mathrm{P}_{2} \mathrm{O}_{5}$ concentrations ( 0.40 to 0.88 wt. \%; columns VIII, XI and XII). However, the $\mathrm{P}_{2} \mathrm{O}_{5}$ was lost in magnetite concentrates obtained by laboratory separation. The relations of $\mathrm{V} / \mathrm{Ni}$ (Fig. 24a) and V/Ti (Fig. 24b) differentiating between magnetite-rich, magmatic ores and iron-formations, are indications that the magnetite ores of Tab. 13 belong to apatite-bearing magmatic iron ores.

The $\mathrm{MnO}-\mathrm{FeO}_{\text {tot }}-\mathrm{Al}_{2} \mathrm{O}_{3}$ diagram (Fig. 25) portrays the whole-rock composition of magnetite-amphibole rocks (Tab. 12, IX to XIII; and Tab. 11, VI to IX), magnetite-chlorite-biotite rocks (Tab. 12, XIV to XVII), and garnetites (Tab. 11, I, II and IV). Plotted are also garnetite-rich rocks, which are however intimately intergrown with magnetite-amphibole rocks (Tab. 11, 
Tab. 11 Whole-rock geochemical compositions (XRF) of hornblendite from Švagrov (I to IV), the Sobotín Massif (Sob) (V; analysis of Fiala et al. 1980), basic dyke in Zámčisko (Zám) (VI; analysis of Fojt et al. 1997), magnetite-amphibole rocks from Švagrov (VII; wet chemical analysis), Sylvani (Sylv) (VIII) and Rudná hora (IX and X), and examples of the host-rock gneiss [Zadní Hutisko (Zadní H) XI, Kyzový důl (K důl) XII, Švagrov XIII].

\begin{tabular}{|c|c|c|c|c|c|c|c|c|c|c|c|c|c|}
\hline & \multicolumn{6}{|c|}{ Metahornblendite } & \multicolumn{4}{|c|}{ Magnetite-amphibole rock } & \multicolumn{3}{|c|}{ Gneiss } \\
\hline & \multicolumn{4}{|c|}{ Švagrov } & \multirow{2}{*}{$\begin{array}{c}\text { Sob } \\
V\end{array}$} & \multirow{2}{*}{$\begin{array}{c}\text { Zám } \\
\text { VI }\end{array}$} & \multirow{2}{*}{$\frac{\text { Švagrov }}{\text { VII }}$} & \multirow{2}{*}{$\begin{array}{l}\text { Sylv } \\
\text { VIII }\end{array}$} & \multicolumn{2}{|c|}{ Rudná hora } & \multirow{2}{*}{$\frac{\text { Zadní H }}{\text { XI }}$} & \multirow{2}{*}{$\frac{\text { K důl }}{\text { XII }}$} & \multirow{2}{*}{$\frac{\text { Švagrov }}{\text { XIII }}$} \\
\hline & I & II & III & IV & & & & & IX & $\mathbf{X}$ & & & \\
\hline $\mathrm{SiO}_{2}$ & 54.80 & 49.80 & 48.80 & 55.30 & 55.41 & 47.42 & 52.70 & 40.30 & 48.90 & 48.70 & 62.20 & 67.40 & 70.30 \\
\hline $\mathrm{TiO}_{2}$ & 0.52 & 1.26 & 2.32 & 0.42 & 0.33 & 2.30 & 0.32 & 0.75 & 0.18 & 0.21 & 0.78 & 0.69 & 0.40 \\
\hline $\mathrm{Al}_{2} \mathrm{O}_{3}$ & 11.15 & 12.90 & 12.30 & 11.40 & 10.24 & 12.70 & 6.40 & 8.69 & 2.40 & 2.80 & 14.80 & 14.10 & 15.10 \\
\hline $\mathrm{Fe}_{2} \mathrm{O}_{3}$ & 2.36 & 4.56 & 6.66 & 3.17 & 2.63 & 4.30 & 23.00 & 16.40 & 28.90 & 29.04 & 4.04 & 2.47 & 0.82 \\
\hline $\mathrm{FeO}$ & 3.39 & 8.23 & 7.40 & 5.42 & 5.27 & 8.44 & 8.80 & 13.70 & 13.30 & 12.41 & 3.62 & 2.99 & 2.59 \\
\hline $\mathrm{MnO}$ & 0.17 & 0.34 & 0.27 & 0.25 & 0.21 & 0.19 & 0.69 & 0.54 & 0.13 & 0.16 & 0.12 & 0.10 & 0.21 \\
\hline $\mathrm{MgO}$ & 4.41 & 8.11 & 8.78 & 10.00 & 9.93 & 7.94 & 2.37 & 8.76 & 1.93 & 2.48 & 2.45 & 1.82 & 1.53 \\
\hline $\mathrm{CaO}$ & 13.10 & 9.36 & 10.45 & 9.08 & 11.19 & 9.58 & 3.07 & 9.52 & 2.56 & 2.89 & 1.65 & 1.69 & 2.52 \\
\hline $\mathrm{Na}_{2} \mathrm{O}$ & 1.83 & 1.03 & 0.86 & 1.96 & 1.53 & 2.31 & 0.61 & 0.91 & 0.14 & 0.32 & 4.87 & 5.01 & 4.33 \\
\hline $\mathrm{K}_{2} \mathrm{O}$ & 0.63 & 0.48 & 0.36 & 0.35 & 0.80 & 0.83 & 0.21 & 0.35 & 0.11 & 0.11 & 2.16 & 1.63 & 1.49 \\
\hline $\mathrm{P}_{2} \mathrm{O}_{5}$ & 5.47 & 0.20 & 0.27 & 0.06 & 0.53 & 0.36 & 0.61 & 0.10 & 0.53 & 0.57 & 0.13 & 0.13 & 0.09 \\
\hline $\mathrm{H}_{2} \mathrm{O}^{+}$ & n.d. & n.d. & n.d. & n.d. & 1.50 & 1.41 & n.d. & n.d. & n.d. & n.d. & n.d. & n.d. & 1.52 \\
\hline $\mathrm{H}_{2}^{-} \mathrm{O}^{-}$ & 0.54 & 0.25 & 0.18 & 0.23 & 0.19 & 0.10 & 0.14 & 0.12 & 0.08 & 0.04 & 0.10 & 0.06 & 0.10 \\
\hline LOI & 1.34 & 1.60 & 1.72 & 1.62 & n.d. & n.d. & 1.27 & 1.12 & 0.67 & 0.83 & 2.13 & 2.09 & n.d. \\
\hline total & 99.71 & 98.12 & 100.37 & 99.26 & 99.76 & 97.88 & 99.37 & 101.26 & 99.83 & 100.56 & 99.05 & 100.18 & 101.00 \\
\hline $\mathrm{Nb}$ & 18 & 37 & 12 & 8 & n.d. & n.d. & n.d. & 14 & 7 & 2 & 19 & 17 & 10 \\
\hline $\mathrm{Zr}$ & 128 & 172 & 152 & 21 & n.d. & n.d. & n.d. & 54 & 63 & 62 & 154 & 139 & 132 \\
\hline $\mathrm{Y}$ & 92 & 32 & 28 & 13 & n.d. & n.d. & n.d. & 18 & 23 & 24 & 23 & 24 & 11 \\
\hline $\mathrm{Sr}$ & 258 & 60 & 202 & 82 & n.d. & n.d. & n.d. & 18 & 13 & 16 & 169 & 198 & 150 \\
\hline $\mathrm{Rb}$ & 17 & 19 & 11 & 14 & n.d. & n.d. & n.d. & $<5$ & 5 & 4 & 85 & 67 & 30 \\
\hline $\mathrm{Pb}$ & 14 & $<10$ & 99 & $<10$ & n.d. & n.d. & n.d. & $<10$ & $<10$ & $<10$ & 11 & $<10$ & 14 \\
\hline $\mathrm{Ga}$ & 14 & 20 & 60 & 14 & n.d. & n.d. & n.d. & 18 & 9 & 7 & 17 & 17 & 19 \\
\hline $\mathrm{Zn}$ & 47 & 63 & 316 & 78 & n.d. & n.d. & n.d. & 115 & 47 & 61 & 56 & 46 & 49 \\
\hline $\mathrm{Ni}$ & 71 & 103 & 107 & 88 & n.d. & n.d. & n.d. & 279 & $<10$ & 18 & 42 & 28 & 36 \\
\hline $\mathrm{Co}$ & 19 & 90 & 88 & 59 & n.d. & n.d. & n.d. & 71 & 40 & 70 & 30 & 22 & 15 \\
\hline $\mathrm{Cr}$ & 40 & 217 & 232 & 960 & 690 & n.d. & n.d. & 541 & 51 & 42 & 60 & 43 & 158 \\
\hline $\mathrm{V}$ & 90 & 194 & 329 & 215 & n.d. & n.d. & n.d. & 171 & 51 & 60 & 108 & 99 & 61 \\
\hline $\mathrm{Ba}$ & 77 & 76 & 40 & 55 & n.d. & n.d. & n.d. & 29 & 35 & 44 & 456 & 425 & 689 \\
\hline Sc & 15 & 28 & 30 & 43 & n.d. & n.d. & n.d. & 43 & 10 & 10 & 16 & 16 & 8 \\
\hline
\end{tabular}

\section{$\mathrm{FeO} \mathrm{O}_{\text {tot }}$}
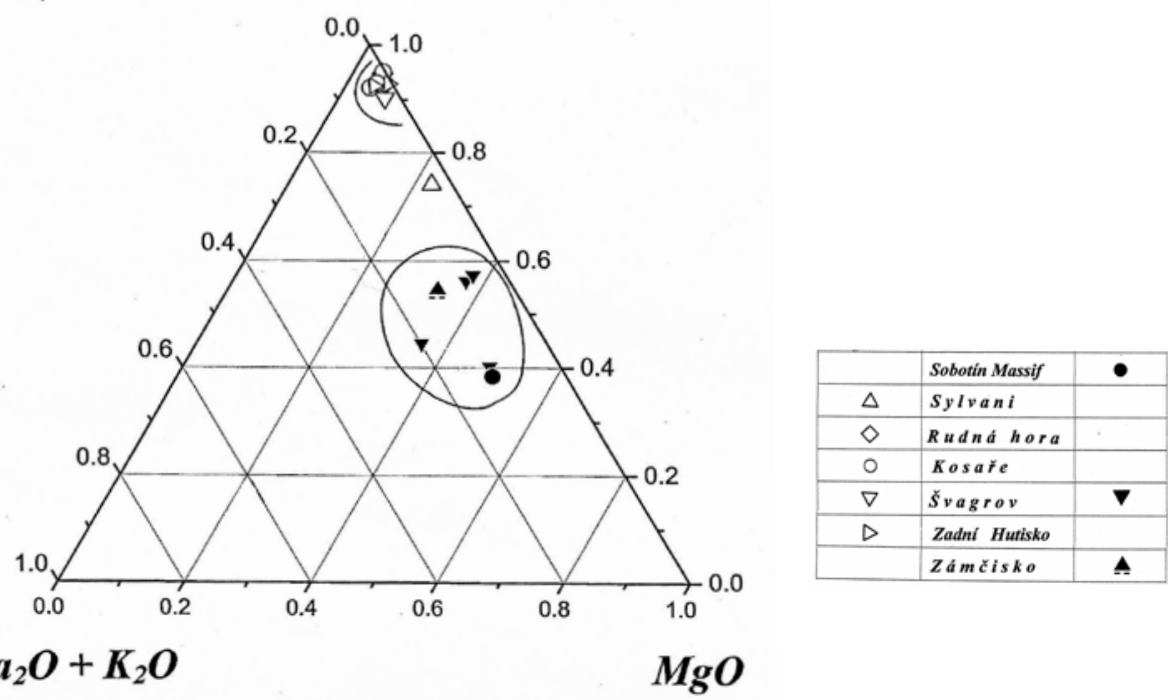

$\mathrm{Na}_{2} \mathrm{O}+\mathrm{K}_{2} \mathrm{O}$

$\mathrm{MgO}$

Fig. 23 The plot $\left(\mathrm{Na}_{2} \mathrm{O}+\mathrm{K}_{2} \mathrm{O}-\mathrm{FeO}_{\text {tot }}-\mathrm{MgO}\right.$ in wt. \%) with fields for the studied magnetite-rich rocks and hornblendites. 


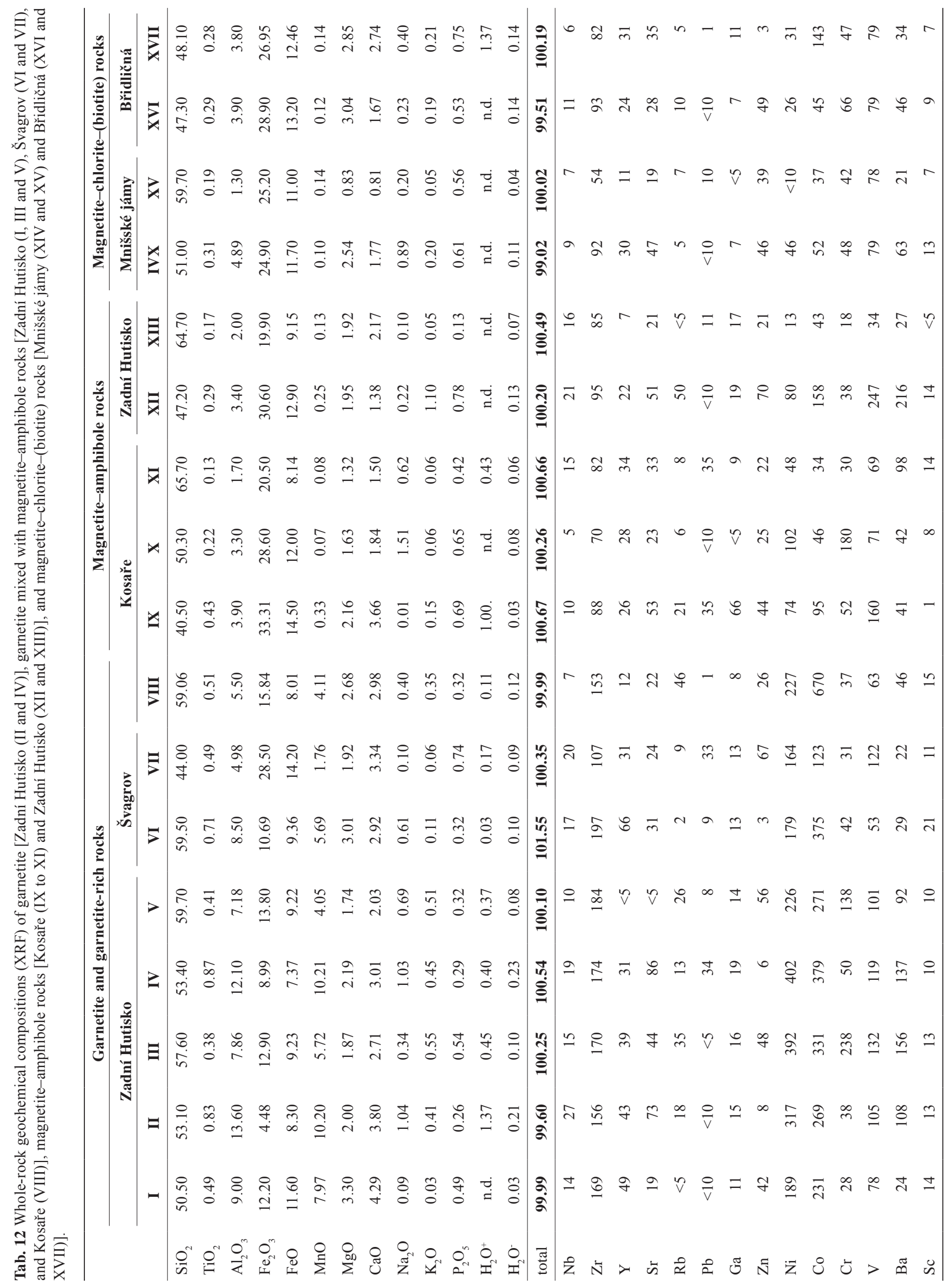



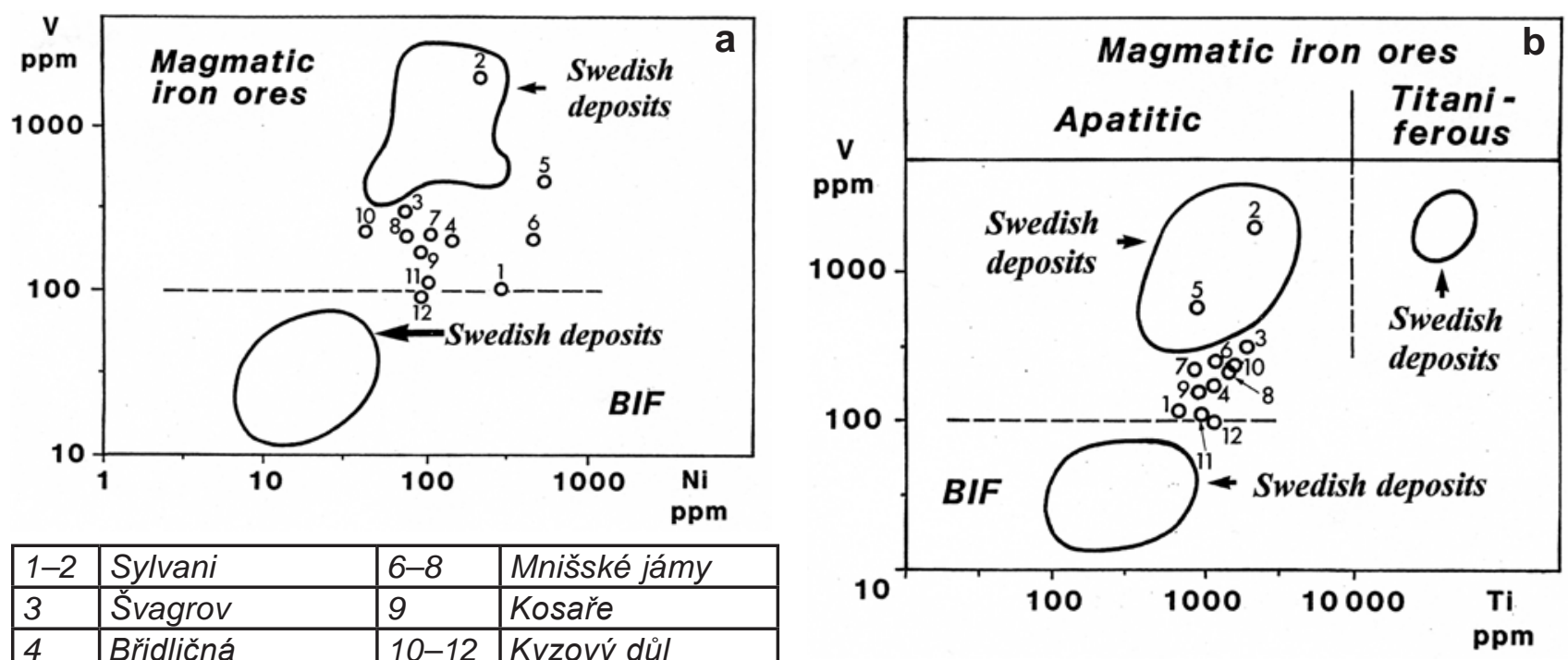

\begin{tabular}{|l|l|l|l|}
\hline $1-2$ & Sylvani & $6-8$ & Mnišské jámy \\
\hline 3 & Švagrov & 9 & Kosaře \\
\hline 4 & Břidličná & $10-12$ & Kyzový důl \\
\hline 5 & Zadní Hutisko & & \\
\hline
\end{tabular}

Fig. 24a - The V/Ni plot after Loberg and Horndahl (1983) for the studied magnetite ores. The diagram distinguishes between magnetite-rich ores of magmatic origin and iron-formations; b - The V/Ti plot after Loberg and Horndahl (1983) for the studied magnetite ores. The diagram distinguishes between magnetite-rich ores of magmatic origin and iron-formations.

Tab. 13 Whole-rock geochemical compositions (XRF) for upgraded magnetite-rich samples (grinding, magnetic separation and treatment in HCl) from Sylvani (I and II), Švagrov (III), Břidličná (IV), Zadní Hutisko (V), Mnišské jámy (VI and VII), Kosaře (IX) and Kyzový důl (X) and compact magnetite samples from Mnišské jámy (VIII) and Kyzový důl (XI and XII). The $\mathrm{Fe}_{2} \mathrm{O}_{3}$ contents represent a difference to the total of $100 \%$.

\begin{tabular}{|c|c|c|c|c|c|c|c|c|c|c|c|c|}
\hline & \multicolumn{2}{|c|}{ Sylvani } & \multirow{2}{*}{$\frac{\text { Švagrov }}{\text { III }}$} & \multirow{2}{*}{$\frac{\text { Břidličná }}{\text { IV }}$} & \multirow{2}{*}{$\frac{\text { Z. Hutisko }}{\mathrm{V}}$} & \multicolumn{3}{|c|}{ Mnišské jámy } & \multirow{2}{*}{$\begin{array}{c}\text { Kosaře } \\
\text { IX }\end{array}$} & \multicolumn{3}{|c|}{ Kyzový důl } \\
\hline & I & II & & & & VI & VII & VIII & & $\mathbf{X}$ & XI & XII \\
\hline $\mathrm{SiO}_{2}$ & 6.40 & 7.30 & 9.40 & 8.50 & 7.60 & 5.50 & 7.90 & 12.40 & 5.20 & 5.10 & 13.90 & 16.80 \\
\hline $\mathrm{TiO}_{2}$ & 0.15 & 0.40 & 0.32 & 0.19 & 0.18 & 0.20 & 0.15 & 0.23 & 0.16 & 0.27 & 0.24 & 0.28 \\
\hline $\mathrm{Al}_{2} \mathrm{O}_{3}$ & 0.30 & 1.02 & 0.96 & 0.78 & 1.08 & 0.45 & 0.45 & 1.94 & 0.45 & 0.57 & 2.43 & 2.32 \\
\hline $\mathrm{Fe}_{2} \mathrm{O}_{3 \text { tot }}$ & 94.47 & 92.85 & 90.33 & 92.01 & 92.35 & 95.87 & 93.85 & 84.73 & 96.74 & 95.78 & 80.88 & 77.54 \\
\hline $\mathrm{Fe}_{2} \mathrm{O}_{3 \text { diff. }}$ & 63.01 & 61.93 & 60.32 & 61.37 & 61.60 & 63.94 & 60.94 & 58.50 & 62.05 & 63.88 & 53.24 & 51.64 \\
\hline $\mathrm{FeO}_{\text {anal. }}$ & 28.31 & 27.82 & 27.00 & 27.57 & 27.67 & 28.73 & 29.61 & 23.60 & 31.21 & 28.70 & 24.87 & 23.30 \\
\hline $\mathrm{MnO}$ & 0.77 & 0.15 & 0.26 & 0.12 & 0.70 & 0.25 & 0.08 & 0.24 & 0.10 & 0.10 & 0.19 & 0.16 \\
\hline $\mathrm{MgO}$ & 0.69 & 1.08 & 0.80 & 0.99 & 0.69 & 0.57 & 0.60 & 1.33 & 0.60 & 0.93 & 2.28 & 2.06 \\
\hline $\mathrm{CaO}$ & 0.22 & 0.16 & 0.60 & 0.25 & 0.28 & 0.13 & 0.15 & 0.54 & 0.15 & 0.36 & 2.13 & 2.44 \\
\hline $\mathrm{Na}_{2} \mathrm{O}$ & $<0.10$ & $<0.10$ & $<0.10$ & $<0.10$ & $<0.10$ & $<0.10$ & $<0.10$ & 0.36 & $<0.10$ & $<0.10$ & $<0.10$ & $<0.10$ \\
\hline $\mathrm{K}_{2} \mathrm{O}$ & 0.03 & 0.03 & 0.03 & 0.06 & 0.06 & 0.06 & 0.06 & 0.30 & 0.03 & 0.04 & 0.09 & 0.12 \\
\hline $\mathrm{P}_{2} \mathrm{O}_{5}$ & 0.03 & 0.02 & 0.13 & 0.08 & 0.05 & 0.08 & 0.06 & 0.40 & 0.05 & 0.05 & 0.63 & 0.88 \\
\hline total & 100.00 & 100.00 & 100.00 & 100.00 & 100.00 & 100.00 & 100.00 & 100.00 & 100.00 & 100.00 & 100.00 & 100.00 \\
\hline $\mathrm{Nb}$ & $<5$ & $<5$ & $<5$ & $<5$ & $<5$ & $<5$ & $<5$ & $<5$ & $<5$ & $<5$ & 15 & 12 \\
\hline $\mathrm{Zr}$ & 72 & 36 & 105 & 114 & 192 & 132 & 156 & 77 & 108 & 99 & 93 & 106 \\
\hline Y & $<5$ & $<5$ & $<5$ & $<5$ & $<5$ & $<5$ & $<5$ & 14 & $<5$ & $<5$ & 30 & 72 \\
\hline $\mathrm{Sr}$ & 21 & 24 & 33 & 24 & 18 & 15 & $<5$ & 1 & 30 & 27 & 42 & 48 \\
\hline $\mathrm{Rb}$ & $<5$ & $<5$ & $<5$ & 15 & $<5$ & $<5$ & $<5$ & 16 & 24 & $<5$ & 15 & 14 \\
\hline $\mathrm{Pb}$ & $<10$ & $<10$ & $<10$ & $<10$ & $<10$ & $<10$ & $<10$ & 28 & $<10$ & $<10$ & $<10$ & 12 \\
\hline $\mathrm{Ga}$ & $<5$ & 30 & 21 & $<5$ & 24 & $<5$ & $<5$ & 7 & $<5$ & $<5$ & $<5$ & 24 \\
\hline $\mathrm{Zn}$ & 42 & 78 & 102 & 39 & 69 & 111 & 39 & 148 & 51 & 54 & 33 & 50 \\
\hline $\mathrm{Ni}$ & 303 & 213 & 72 & 132 & 570 & 150 & 102 & 80 & 92 & 45 & 99 & 98 \\
\hline Co & 105 & 219 & 168 & 93 & 672 & 84 & 90 & 72 & 72 & 93 & 150 & 36 \\
\hline $\mathrm{Cr}$ & 69 & 108 & 90 & 87 & 99 & 69 & 99 & 54 & 54 & 60 & 42 & 26 \\
\hline V & 114 & 1179 & 264 & 168 & 477 & 222 & 216 & 191 & 156 & 192 & 108 & 88 \\
\hline $\mathrm{Ba}$ & 57 & $<10$ & 36 & 42 & 81 & 60 & 87 & 65 & 60 & $<10$ & 51 & 20 \\
\hline $\mathrm{Sc}$ & 15 & $<5$ & $<5$ & $<5$ & $<5$ & $<5$ & 15 & 25 & 21 & $<5$ & 21 & 5 \\
\hline
\end{tabular}




\begin{tabular}{|c|l|}
\hline$\triangle$ & Sylvani \\
\hline$\diamond$ & Rudná hora \\
\hline$\bigcirc$ & Kosaře \\
\hline$\nabla$ & Švagrov \\
\hline$\triangleright$ & Zadni Hutisko \\
\hline$\downarrow$ & Břidličná \\
\hline$\triangleleft$ & Mnišské jámy \\
\hline
\end{tabular}

Fig. 25 Ternary plot $\mathrm{MnO}-\mathrm{FeO}_{\text {tot }}-\mathrm{Al}_{2} \mathrm{O}_{3}$ (wt. \%) for the magnetite-rich rocks and garnetites from Desná Dome. The field of coticules defined by Spry et al. (1997) is also shown in Fig. 25. The point marked by arrow (Švagrov; Tab. 10, column VII) represents an amphibole-magnetite rock, which contains minor admixture of garnetite.

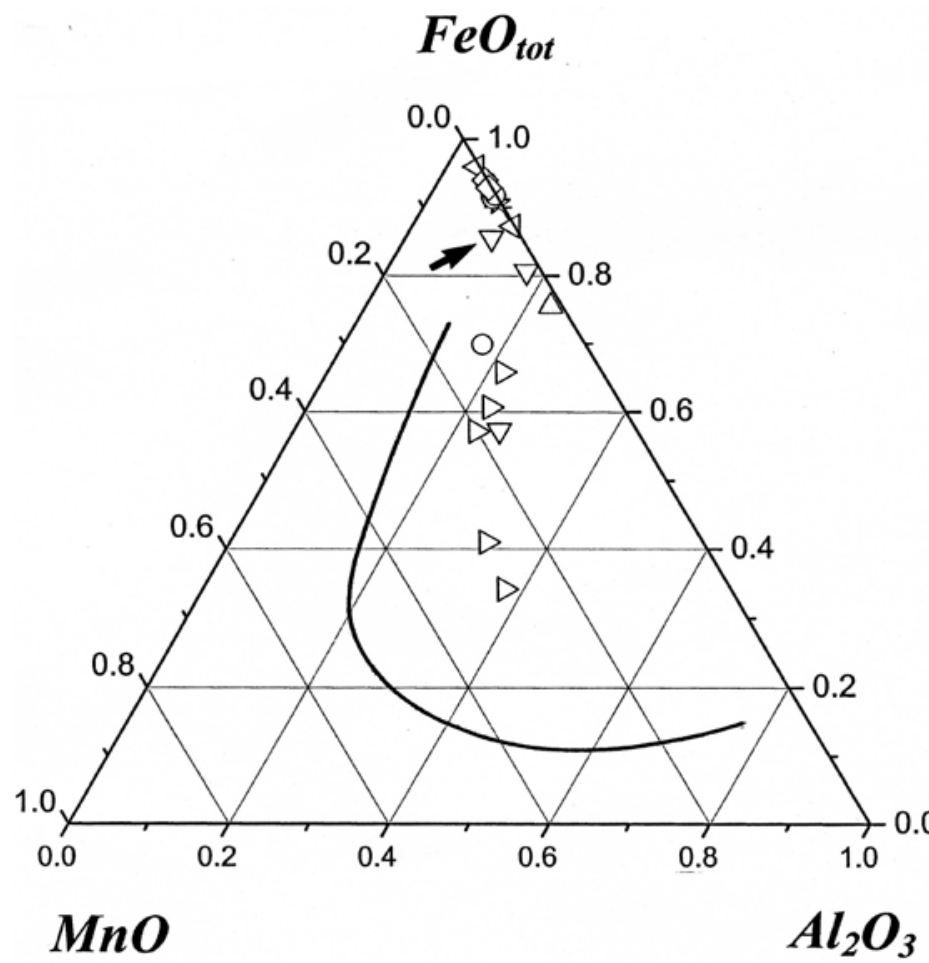

of the basic rocks of the Sobotín Massif. In relation to the other rocks, compact magnetite (line IV) is interpreted to be of late stage origin. It is slightly more differentiated than the other samples $(\mathrm{LREE} / \mathrm{HREE}=8.1$; highlighted in Fig. 26).

Figure 27 contains exclusively rocks from Zadní Hutisko (I to III; Tab. 14). The pattern with the highest REE concentration (347.13 ppm), represents data for garnetite with more than 10 wt. \% MnO (Tab. 12, II). The pattern with the lowest total REE contents corresponds to the host-rock gneiss. The remaining sample is a mixture of the two. The Ce anomaly of the garnetiferous rock probably reflects the input of detrital material during the formation of the protolith. The garnetite pattern is nearly identical to the REE normalized patterns from the Joffre iron-formation (Hamersley Range, Australia), containing a significant detrital component (Alibert and McCulloch 1993).

\subsection{Amphibole}

Unlike in the studied Desná rocks, amphiboles of ironformations always have $\mathrm{Mg} /\left(\mathrm{Mg}+\mathrm{Fe}^{2+}\right)$ values lower than 0.4 and generally correspond to the $\mathrm{Fe} / \mathrm{Mg}$-amphibole grunerite. Exceptionally, ferroactinolite and rarely ferrohornblende and ferrotschermakite can occur (Mücke 2003). The investigated amphiboles of the magnetite-amphibole rocks and the hornblendites are exclusively calcic with $\mathrm{Ca} \geq 1.50$ and $(\mathrm{Na}+\mathrm{K})_{\mathrm{A}}<0.50$ (Tabs 2 and may reflect a low oxygen fugacity after the emplacement 


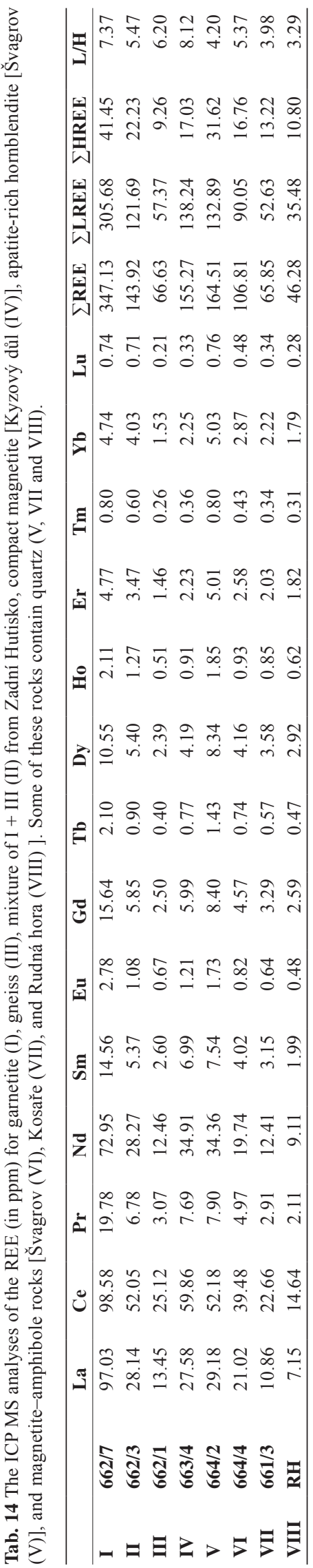

$661 / 3 * 663 / 4 \diamond 664 / 2 \triangle 664 / 4 \diamond \mathrm{RH}$

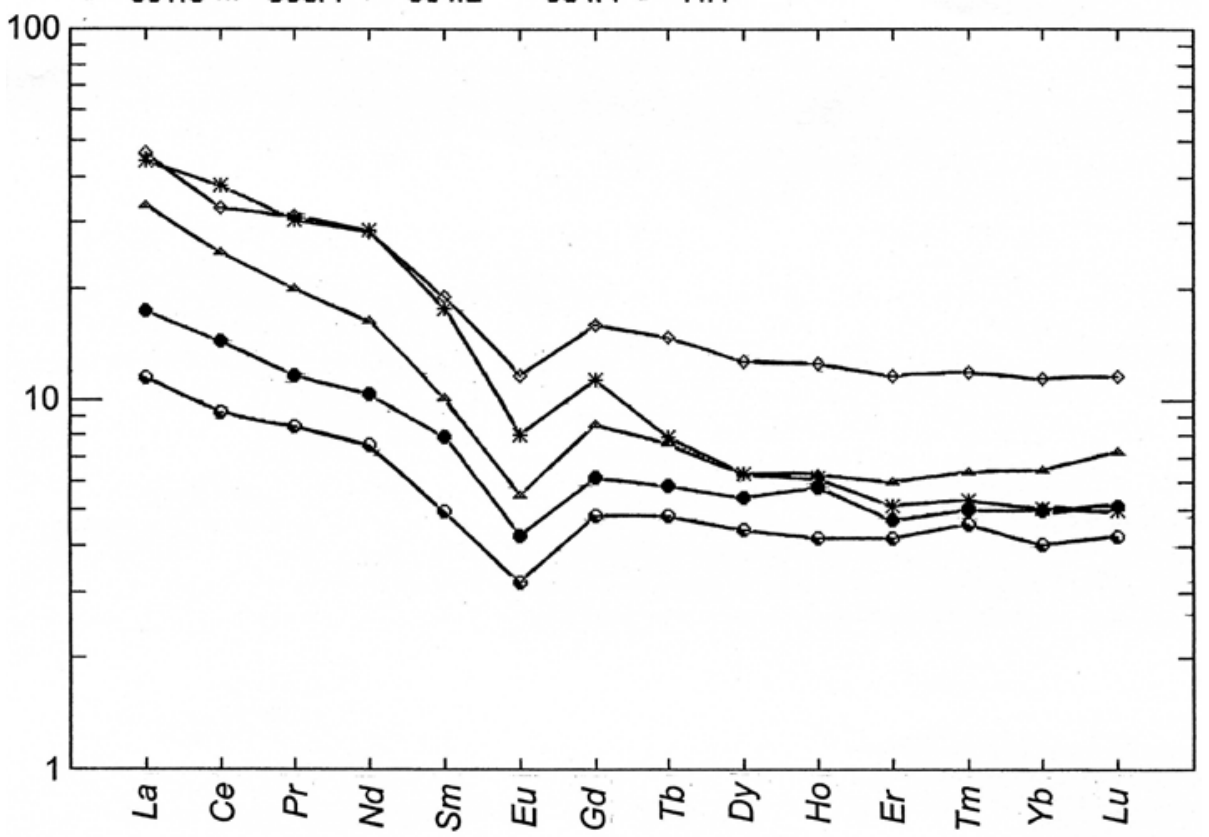

Fig. 26 Chondrite-normalized REE diagram for 5 samples (663/4 - compact magnetite, Kyzový důl; 664/2 - apatite- and quartz-rich hornblendite with amphibole relics and high content of newly-formed epidote, Švagrov; 664/4 - Amphibole-magnetite rock, Švagrov; 661/3 - quartz-rich amphibole-magnetite rock which also contains epidote and apatite, Kosaře; and RH - alternate layers of amphibole-magnetite rock and quartz, Rudná hora.

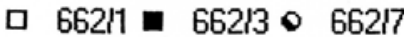

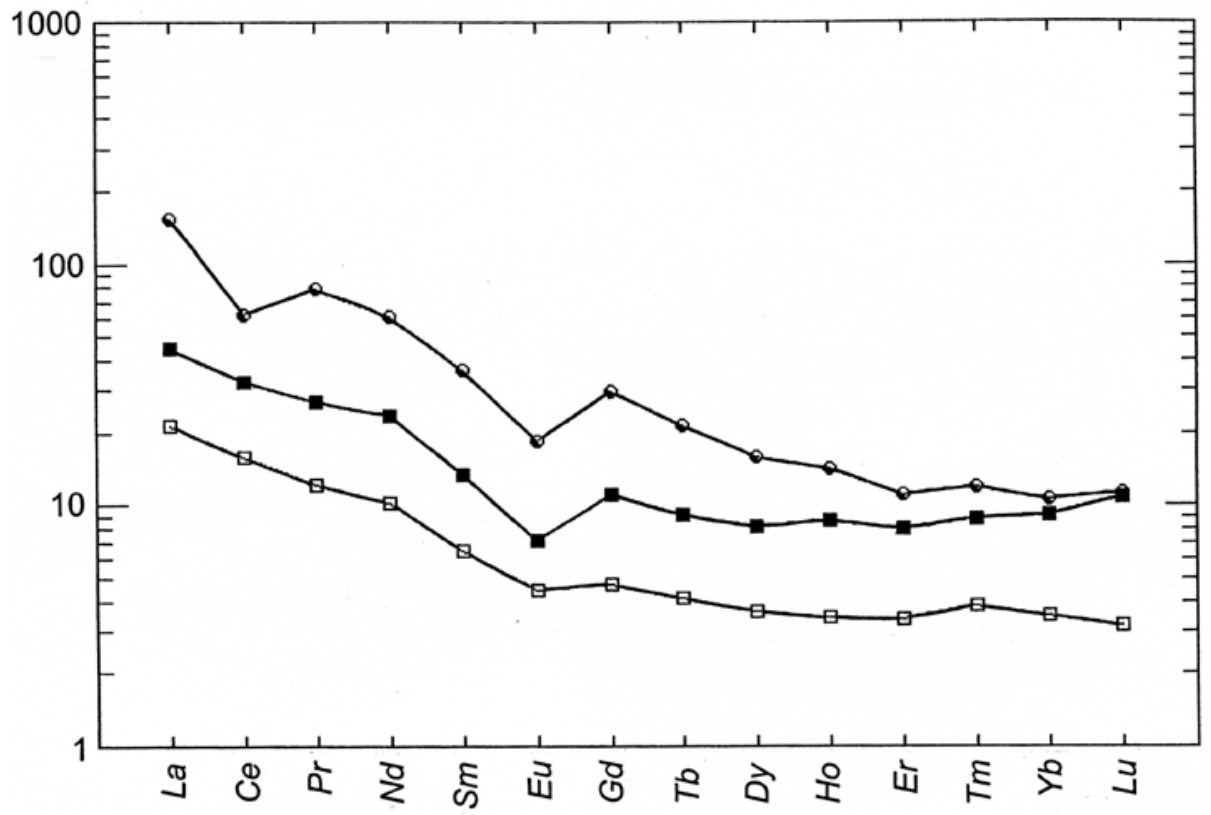

Fig. 27 Chondrite-normalized REE plot for three samples from Zadní Hutisko (662/7: garnetite; 662/1: host-rock gneiss; and 662/3: mixture of garnetite and gneiss) 
3). The analytical points are predominantly located in a small area of Fig. 16 with $\mathrm{Mg} /\left(\mathrm{Mg}+\mathrm{Fe}^{2+}\right)=0.6$ to 0.8 and $\mathrm{Si}=6.0$ to 7.8 , i.e., along the boundary between the tschermakite and magnesiohornblende domains. Only a few analyses have higher Si contents, reaching up to actinolite. The latter come from amphiboles near the rim of amphibole crystals or amphibole relics within quartz-rich rocks (mainly from Kosaře and Zadní Hutisko). Three analyses have higher contents of iron and correspond to ferrohornblende (Zámčisko; analyses of Fojt et al. 1997).

In his study, Pouba (1970) found that amphiboles occurring within apophyses (genetically related to the Sobotín Massif) and magnetite bands (belonging to ironformations) are of the same composition. Now the results of Pouba (1970) can be expanded, because there seem to be no compositional differences between amphiboles, which occur in rocks (magnetite-amphibole rocks and metahornblendite) included within the gneisses of the Desná Group and in those of the Sobotín Massif itself (including Rudná hora and Sylvani). Therefore, the discussion of the data in Fig. 16 leads to the following conclusions:

- there is no indication that the amphiboles originated from more than one source; and

- the amphiboles have compositions that do not occur in iron-formations.

In summary it can be concluded that the amphiboles have probably been derived from a magmatic protolith and were genetically related to the Sobotín Massif. This view is supported by investigations of Cháb et al. (1984), who believed that amphibolites and metagabbros occurring within the Desná gneisses formed from intrusive rocks associated with the Sobotín Massif.

It is assumed that the original amphibole compositions were tschermakite and magnesiohornblende. Amphiboles with higher Si contents, including actinolite, most likely reflect secondary alteration (during metamorphism). Amphiboles at Zámčisko have the highest Fe content (analyses of Fojt et al. 1997), and are ferrohornblendes. It is inferred that the dykes from Zámčisko are connected with the first emplacement of the apophyses within the gneisses.

\subsection{Biotite}

Figure 17 shows that the biotites are dominated by the phlogopite end-member (c. 65 to $50 \mathrm{~mol} . \%$ ) and their $\mathrm{Al}$ content, expressed by the ratio of $\left(\mathrm{AlSi}_{3}\right):\left(\mathrm{Al}_{1.5} \mathrm{Si}_{2.5}\right)$, is not higher than $1: 1$ and has a minimal value of $9: 1$ (Kyzový důl). Similar to amphiboles, the highest Fe contents in biotite were found at Zámčisko (Tab. 4, VIII; analyses of Fojt et al. 1997). The lowest iron content of biotite occurs in amphibole-magnetite rocks at Švagrov (with the highest $\mathrm{Al}$ concentration) as well as in magnetite-chlorite-(biotite) rocks in Zadní Hutisko (one sample) and Kyzový důl (one sample), which have the lowest $\mathrm{Al}$ contents. The biotite from the host-rock gneiss seems compositionally undistinguishable from that in the other rocks studied.

\subsection{Chlorite}

The chlorite compositions, summarized in Fig. 18 and Tab. 5, are characterized by the presence of c. $50 \mathrm{~mol}$. \% of the $\mathrm{Mg}$ end-member (38.6-60.6 mol. \%, but mainly 48-54 mol. \%), c. 28 mol. \% $\mathrm{Fe}_{\text {tot }}$ (21.9-44.7, mostly 25-31 mol. \%) and c. $22 \mathrm{~mol} . \% \mathrm{Al}^{\mathrm{VI}}$ (16.7-28.4 mol. \%, mostly $20-25$ mol. \%). Additionally, Fig. 18 shows that the chlorite analyses from the Sobotín Massif have the highest $\mathrm{Mg}$ values (58.4-68.12 mol. \%) and those of the host rock gneiss relatively low $\mathrm{Mg}$ concentrations (45.0-47.2 mol. \%). Due to the fact that many minerals of the chlorite group were recently abolished (e.g., thuringite and delessite), the $\mathrm{Fe}_{\text {tot }} /\left(\mathrm{Fe}_{\text {tot }}+\mathrm{Mg}\right)-\mathrm{Si}$ diagram of Melka (1965) was modified to discriminate between clinochlore and chamosite only (Fig. 19). Here again it is obvious, that the chlorites occupy a relatively small compositional field and are exclusively clinochlores. Figure 19 also shows that chlorite of magnetite-rich rocks cannot be differentiated from chlorite of the gneisses, but both groups are generally richer in iron than chlorites of hornblendites.

Chlorite of iron-rich rocks originated either by the replacement of amphibole (Fig. 13b) or of biotite (Fig. 13f). As chloritization affects predominantly the biotite rather than amphibole, chlorite is an abundant mineral in parageneses that were originally rich in biotite (Figs 14b and 14d). Figures 18 and 19 do not discriminate well between chlorites that occur in the host-rock gneiss or in the other rocks.

Compositional differences become somewhat clearer by using the $\mathrm{Al} /(\mathrm{Al}+\mathrm{Fe}+\mathrm{Mg}) \mathrm{vs} . \mathrm{Mg} /(\mathrm{Mg}+\mathrm{Fe})$ plot of Liard (1988), modified by Mücke (2006) (Fig. 28). This is useful for illustrating differences in compositions of chlorites that were formed in defined environments (e. g. felsic, mafic, ultramafic). The chlorites are mostly concentrated within a small area of the diagram shown in Fig. 28. In contrast to the Figs 19 and 20, chlorites of the host gneisses are slightly separated from those of the other rocks.

For this study, the most important information spanning from Fig. 28 is that the investigated chlorites do not correspond to chlorites of iron-formations as postulated by Pouba $(1960,1970)$ and Fojt $(2002,2003)$. Clearly their $\mathrm{Mg} /(\mathrm{Mg}+\mathrm{Fe})$ values $(0.55-0.73)$ are high, characteristic of mafic rocks, rather than chlorites in iron-formations $(\mathrm{Mg} /(\mathrm{Mg}+\mathrm{Fe})=0.15$ to 0.43 , Liard 1988). 


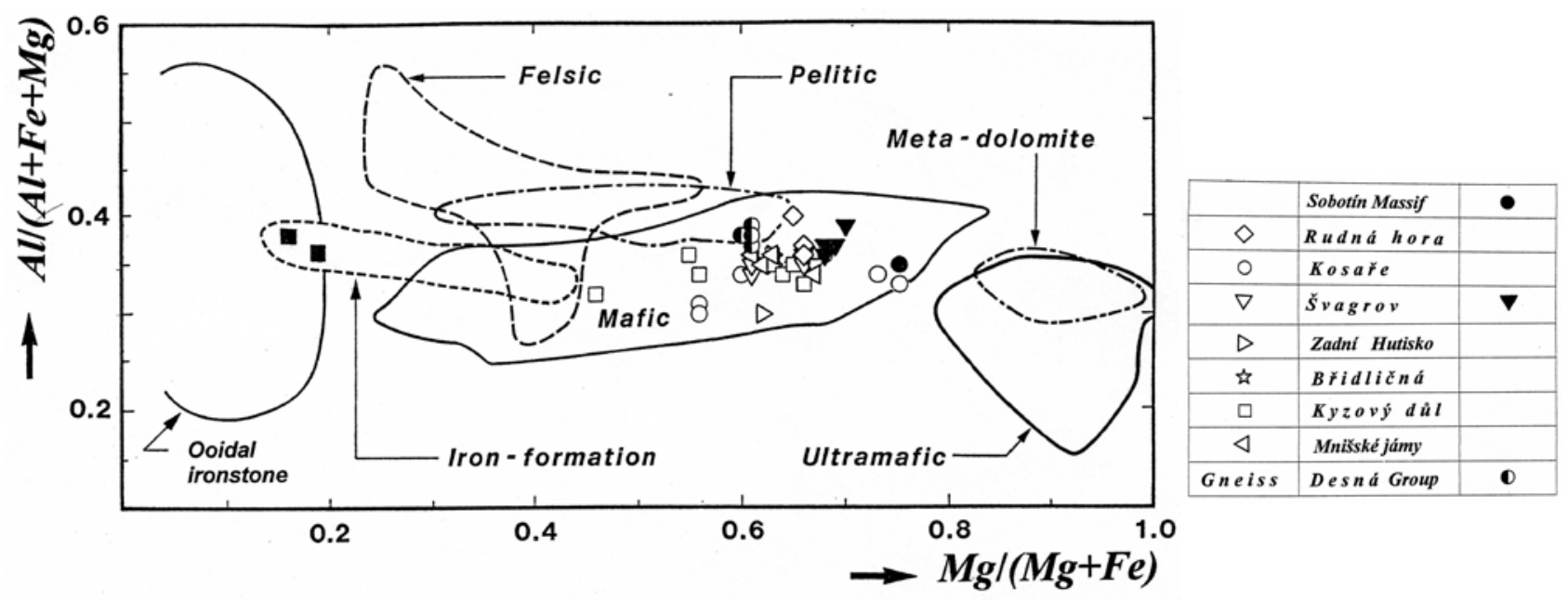

Fig. 28 Chlorite classification diagram $(\mathrm{Mg} /(\mathrm{Mg}+\mathrm{Fe})$ vs. $\mathrm{Al} /(\mathrm{Al}+\mathrm{Fe}+\mathrm{Mg})$ in mol. \%), modified from Liard (1988). Plotted are: 1 - amphibole-magnetite rocks, 2 - magnetite-chlorite-(biotite) rocks falling predominantly into the "mafic" field, 3 - gneisses [plotting in the pelitic field, close to the rocks mentioned under (1) and (2)], and 4 - the Lahn-Dill type deposit of the Malý Děd Mt. (filled squares, the field of iron-formations).

\subsection{Garnet}

Generally, the garnetites occur as thin bands (Zadní Hutisko, Švagrov, Kosaře, in part also Mnišské jámy) alternating with quartz and/or magnetite-rich layers. However, in Kyzový důl, the garnetite sensu stricto is not preserved as it is widely replaced by late quartz, in which only garnet relics remain. This is in part similar for garnetite of Mnišské jámy (Fig. 14e).

Figure 20 demonstrates that the almandine and spessartine contents are nearly identical at Kosaře, Švagrov and Mnišské jámy [Tab. 6: almandine 38.45-49.85 mol. \% (V and IV) and spessartine 25.35-33.47 mol. \% (XIII and V)]. Zadní Hutisko contains garnet with the highest spessartine content [45.65-63.70 (XI and X), on average 51.94 mol. \% (XII)]. Exceptional are the garnets from Kyzový důl. They have the highest contents of the other end-members (grossular + andradite + pyrope range from 31.1-34.0 mol. \%; VII and VIII) and therefore relatively low spessartine (on average $38.85 \mathrm{~mol} \%$, IX) and almandine (on average $28.67 \mathrm{~mol}$. \%, IX) contents.

Compared to the other end-members (pyrope and andradite), Fig. 21 shows that garnets of Mnišské jámy are relatively enriched in the grossular component. On the other hand, some garnets (Švagrov and Zadní Hutisko) have high andradite and pyrope relative to grossular.

The geological position of garnetite is rather controversial. Pouba (1960 and 1970), together with Zimák and Kropáč (2006) were of the opinion that the magnetite-rich rocks and garnetites occurring within the Desná gneisses are both of the same age and identical with iron-formations. Fojt (2002), on the other hand, was convinced that the garnetites are the youngest within the sequence. However, the petrographic investigations of the garnetites and the associated magnetite-rich rocks clearly show that the garnetites are older. This follows from the fact that the garnet crystals in contact with magnetite-amphibole bands are overgrown especially by amphibole (Figs 12f, $13 \mathrm{a}$ and 15d) and magnetite (Fig. 13a) and in contact with magnetite-chlorite-(biotite) rocks, by chlorite or biotite (Fig. 14f).

Apart from the results of the petrographic observations, there is another important argument against the interpretation of Pouba (1960 and 1970). This follows from the close association of manganese-rich garnet bands in the $\mathrm{mm}$ to $\mathrm{cm}$ range (with $\mathrm{MnO}$ concentrations up to 10 wt. $\%$ and $\mathrm{Fe}_{2} \mathrm{O}_{3 \text { tot }}$ c. 15 wt. \%; Tab. 12, columns II and IV) with bands consisting of amphibole and magnetite (with 20 to 33 wt. $\% \mathrm{Fe}_{2} \mathrm{O}_{3}$ and 8 to 14.5 wt. $\% \mathrm{FeO}$, as well as negligible $\mathrm{Mn}$ contents, $\mathrm{MnO}<0.33$ wt. \%; Tab. 12, IX to XIII). These compositionally contrasting rocks require separation of $\mathrm{Fe}$ and $\mathrm{Mn}$ during sedimentation. This was explained by Pouba (1970) in terms of zoning within the Proterozoic sea, in which the elements were presumably heterogeneously distributed: Fe-rich sediments could have been deposited near the shore, whereas those rich in $(\mathrm{Mn}+\mathrm{Fe})$ occurred in a deep sea.

The separation of $\mathrm{Fe}$ and $\mathrm{Mn}$ in ore deposits has been discussed in the literature for more than 50 years and various models emerged (e.g. Krauskopf 1957; Glasby 1997; Bühn and Stanistreet 1997). For example, in the Kalahari manganese field, layers of iron-formation are interbedded with manganese ore beds. Beukes and Guzmer (1996) were of the opinion that the latter resulted from strong hydrothermal alteration of the iron-formation beds, resulting in the formation of newly-grown Mn-enriched minerals that do not occur in the unaltered rocks. Klemm (2000), who studied the same manganese field, explained 
the separation of $\mathrm{Fe}$ and $\mathrm{Mn}$ on the basis of physicochemical conditions of the sea water. Whereas iron precipitated in the form of $\mathrm{Fe}_{2} \mathrm{O}_{3}$ (pH 7 to 8 and $\mathrm{Eh} 0$ to -0.2 ), manganese precipitation was expected to occur in an environment characterized as organic-rich saline water by Baas Becking et al. (1960). In such an environment, high $\mathrm{pH}$ values, even exceeding 10 , can be achieved. These physico-chemical conditions occurred most likely by closing the access to the open ocean, which resulted in the reduction of the water level by evaporation.

The scenarios discussed above may be envisaged for rock sequences with alternating layers in the order of some 10 meters and more, similar to the Kalahari manganese field. However, in settings similar to the rocks of our study having alternating $\mathrm{Mn}$ - and $\mathrm{Fe}$-rich bands in the $\mathrm{cm}$-range only, the Kalahari model cannot be applied. This is considered as an additional evidence that the Mn-rich rocks within the Desná gneisses were not formed simultaneously with the associated iron mineralization, but are older, as indicated by petrographic observations. This view agrees with investigations of Žák $(1974,1975)$, who found that the garnetites (of Švagrov) are older than the magnetite-rich rocks.

\subsection{Magnetite}

Magnetite was analysed in samples of magnetite-amphibole rocks of the Sobotín Massif (Sylvani and Rudná hora; Tab. 7, I-III); magnetite-amphibole rocks of Kosaře and Švagrov (VI, IX and X) and magnetite-chlorite-(biotite) rocks of Zadní Hutisko (IV and V), Kyzový důl (VII and VIII), and Mnišské jámy (XI and XII), the latter two rock types occurring within the Desná gneisses. Rocks consisting exclusively of magnetite and quartz were not observed. Ulvite component ranges between 0.10 and $0.90 \mathrm{~mol}$. \% (up to $2.50 \mathrm{~mol}$. \%, Mnišské jámy, XII). Jacobsite proportions vary from 0.20 to $0.80 \mathrm{~mol} . \%$ and the highest value was obtained in Sylvani $(2.20 \mathrm{~mol} . \%$, II). Hercynite contents are 0.10 to 0.65 mol. $\%$, whereas the other end-members, not present in all the magnetite samples (or not analysed), show the following maxima: magnesioferrite 0.40 mol. \% (Sylvani, column I), trevorite $0.40 \mathrm{~mol}$. \% (Zadní Hutisko, IV) and chromite 0.15 mol. \% (e.g., Mnišské jámy, XII).

It was shown above that magnetite-rich rocks are most probably of magmatic origin and consequently of the same age and origin (magmatic) as amphibole. This corresponds to the mode of occurrence of the two minerals, magnetite and amphibole, as intimate intergrowths (Figs 12a, 12b and 15b).

\subsection{Ilmenite}

Characteristic feature of the ilmenite solid solutions, presented in Tab. 8, is the relationship between $\mathrm{FeTiO}_{3}$ and $\mathrm{MnTiO}_{3}$ components ranging from 79.9 mol. \% $\mathrm{FeTiO}_{3} / 9.4$ mol. \% $\mathrm{MnTiO}_{3}$ (Sylvani, III) to $44.2 \mathrm{~mol} \%$ \% $\mathrm{FeTiO}_{3} / 52.5$ mol. \% $\mathrm{MnTiO}_{3}$ (Mnišské jámy, XVI). Contrary to this broad compositional span, the variation of these two end-members at any single location is relatively low: for instance at Sylvani $\left(\mathrm{FeTiO}_{3}: 65.5\right.$ to 79.9 mol. \%; $\mathrm{MnTiO}_{3}: 23.9$ to $9.4 \mathrm{~mol}$. \%) or at Mnišské jámy $\left(\mathrm{FeTiO}_{3}\right.$ : 44.2 to $46.2 ; \mathrm{MnTiO}_{3}: 52.5$ to $46.9 \mathrm{~mol}$. \%). Other endmembers occur only sporadically: perovskite is restricted to Kosaře ( 0.4 mol. \%, I), Švagrov ( 0.3 to $2.4 \mathrm{~mol}$. \%, V and VI) and Zadní Hutisko (0.2 to $0.3 \mathrm{~mol}$. \%, XIII and XIV), eskolaite was recorded from Sylvani and Kyzový důl (0.05 mol. \%, II and VIII). The $\mathrm{Fe}_{2} \mathrm{O}_{3}$ end-member is always present and its amount varies between 1.2 (Kyzový důl, IX) and 10.5 mol. \% (Sylvani, II). The high $\mathrm{Fe}_{2} \mathrm{O}_{3}$ content of 18.6 mol. \% in Kosaře (I) is an exception and most probably of secondary origin. This is the reason why the latter data were not incorporated in Fig. 29, illustrating the variation of $\mathrm{FeTiO}_{3}$ and $\mathrm{MnTiO}_{3}$ contents in the ilmenite solid solution.

The analytical data were obtained from ilmenite of magnetite-rich rocks showing that the manganese concentration increases steadily at the expense of the iron from Sylvani to Mnišské jámy. The relationships in Fig. 29 are not accidental but may be explained by assuming magmatic rocks as a protolith. Most probably the formation of ilmenite solid solutions was associated with basic rocks of the Sobotín Massif. The whole rocks have low $\mathrm{Mn}$ abundances and, therefore, independent Mn minerals do not occur. Upon continuous cooling, the manganese concentration in ilmenite increases with decreasing temperature (Hagerty 1976). This is the reason why the solid solutions between $\mathrm{FeTiO}_{3}$ and $\mathrm{MnTiO}_{3}$ become steadily richer in the pyrophanite $(\mathrm{Mn})$ end-member up to amounts that are higher than $\mathrm{FeTiO}_{3}$, an observation also noted by Ramdohr (1975). The highest pyrophanite content was analysed at Mnišské jámy (on average 50.1 mol. \% $\mathrm{MnTiO}_{3}$; Tab. 8, XVIII; Fig. 29), the deposit most remote from the Sobotín Massif (Fig. 1).

In other rocks the relationship illustrated in Fig. 29 was not observed:

- Ilmenite in paragenesis with garnet (Zadní Hutisko) has higher Mn concentrations than in the associated magnetite-rich rocks, ranging between 67.3 and 69.2 mol. \% $\mathrm{MnTiO}_{3}$ (28.7 and 30.4 mol. \% $\mathrm{FeTiO}_{3}$; data calculated from electron-microprobe analyses which are not included in Tab. 8). Ilmenite at the same locality and in iron-rich rocks contains on average $50.3 \mathrm{~mol}$. \% $\mathrm{FeTiO}_{3}$ and $47.0 \mathrm{~mol}^{\%} \mathrm{MnTiO}_{3}$ (Tab. 8, XV);

- Ilmenite occurring in hornblendite of Švagrov with magnetite as an accessory constituent is nearly pure $\mathrm{FeTiO}_{3}$ having on average $3.6 \mathrm{~mol} . \% \mathrm{MnTiO}_{3}$ only (Tab. 8, XIX). Ilmenite in amphibole-magnetite rocks at the same locality contains on average $69.1 \mathrm{~mol} . \%$ $\mathrm{FeTiO}_{3}$ and $24.5 \mathrm{~mol} \% \mathrm{MnTiO}_{3}(\mathrm{VII})$. 


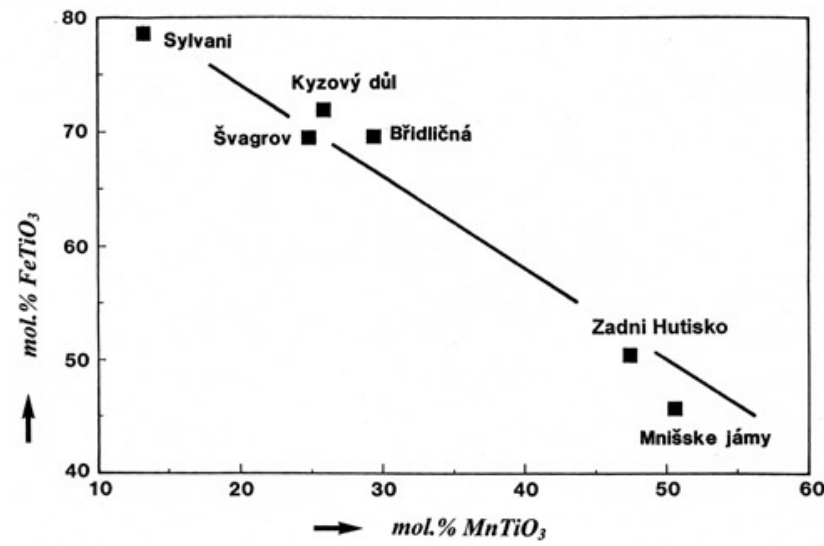

Fig. 29 Composition of ilmenite solid solutions in the binary plot $\mathrm{MnTiO}_{3}$ vs. $\mathrm{FeTiO}_{3}(\mathrm{~mol} . \%)$.

\subsection{Apatite}

Apatite, identified as nearly pure fluorapatite, is a characteristic constituent of all the studied iron-rich rocks and is mainly homogeneously distributed (on average $0.5 \mathrm{wt}$ \% $\mathrm{P}_{2} \mathrm{O}_{5}$ ) but may locally be enriched up to about $5.5 \mathrm{wt}$. \% $\mathrm{P}_{2} \mathrm{O}_{5}$ (Tab. 11, I).

In iron-formations, apatite is an unusual mineral, and the whole-rock $\mathrm{P}_{2} \mathrm{O}_{5}$ contents are generally below 0.10 wt. \% (Mücke 2003). However, in magmatic rocks, apatite is a common constituent, especially in basic, magnetite-rich types. As the $\mathrm{F} / \mathrm{Cl}$ ratio in apatite increases with decreasing temperature (Korzhinsky 1982), the amount of $\mathrm{Cl}$ and $\mathrm{F}$ in magmatic apatite varies depending on the position within the deposit, and thus the temperature of formation. In general, the F-content in magmatic apatite is distinctly higher than that of $\mathrm{Cl}$. In the Tjårrojåkka apatite-iron deposit, Kiruna, Sweden, it was observed that the F content in apatite increases (up to pure fluorapatite with minor (OH) concentrations) with distance from the ore body (Edfelt et al. 2005).

The composition, the big grain sizes and local enrichments of apatite in some rocks are considered as indications of a magmatic origin. The enrichment is interpreted by magmatic segregation, mainly, but not exclusively, in association with magnetite. As apatite is a relatively refractory mineral, it is suggested that it escaped metamorphic recrystallization.

\subsection{Feldspar and carbonate}

From their close association and intergrowths it is concluded that albitic feldspar $\left(\mathrm{An}_{86.8-98.96}\right)$, chlorite, carbonate [mainly $\mathrm{CaCO}_{3}$ (ranging from 89.24 to $99.13 \mathrm{~mol}$ \% with subordinate $\mathrm{FeCO}_{3}, \mathrm{MnCO}_{3}$ and $\mathrm{MgCO}_{3}$ ), rarely ankerite-rich dolomite] and epidote are of the same age and are newly-formed, replacing pre-existing minerals.
More calcic plagioclase $\left(\mathrm{An}_{23.58-51.45}\right)$ was found in metahornblendites at Švagrov and Zadní Hutisko.

\subsection{Quartz}

Quartz occurs in all the rocks and in varying proportions. With increasing abundance of quartz, the planar fabric (foliation and schistosity) becomes more prominent, a phenomenon that was also observed by Pouba (1970). This author stated that with increasing quartz content, the layering becomes obvious and, in the presence of magnetite, a banded structure develops. The reason for this seems the fact that nearly all minerals mentioned above were subject to silicification. The pre-existing minerals of all rock types were replaced by solutions from which $\mathrm{SiO}_{2}$ precipitated in the form of quartz. These solutions penetrated the rocks using in particular the pre-existing planes of schistosity and foliation. The result is an alternation of light and dark bands (Figs 3, $4,7,8$ and $15 \mathrm{c}$ ). The dark bands represent probably the original rock, whereas the light ones are dominated by late quartz. These two types of bands are tied by continuous transitions. Along the contact occurs a small zone along which the minerals of the dark bands (amphibole, chlorite, biotite and magnetite) were corroded (Figs 12c, $13 \mathrm{a}$, and $15 \mathrm{c}$ ). In a direction perpendicular to the layer, the amount of corroded mineral relics decreases and finally pure quartz emerges in the central part of the quartz bands.

In carbonate-rich rocks and along the contact of carbonate with quartz, it can be observed that the carbonate grain boundaries are strongly corroded (Fig. 14c) indicating that the quartz is younger than the carbonate.

The temperature of silicification may be deduced from reactions observed along the contact garnetite/quartz (Fig. 15a). In the upper part of Fig. 15a, the fine-grained garnetite passes relatively abruptly into late quartz. In a small zone occur garnet crystals, which are not only bigger, but also corroded. The small garnet crystals of the garnetite exhibit only limited compositional variations (Tab. 15, compare I and II), whereas the bigger garnet crystals within late quartz are zoned (Tab 15, compare III with IV and V). Therefore, the influence of late quartz on garnet does not only cause recrystallization (due to coarsening; Fig. 15a) and corrosion (due to irregular outlines; Fig. 15a), but also the variations of the chemical composition of the crystals. Comparing III of Tab. 15 (centre) with IV and V (rim of the crystals) it becomes obvious that the almandine content decreases and spessartine is relatively enriched rimwards. These changes, recrystallization, stabilization of spessartine-rich garnet and its corrosion indicate temperatures which may be close to the temperature of spessartine formation (about $400^{\circ} \mathrm{C}$; Matthes 1961). Therefore, it can be inferred that 
Tab. 15 Garnet end-member proportions (mol. \%) calculated from electron-microprobe analyses of the sample portrayed in Fig. 15a. Tiny garnet grains of garnetite (I and II) and coarse-grained, corroded garnet crystals parallel to the contact (III: center of the crystals, IV and V: rim of the crystals).

\begin{tabular}{lrrrrrr}
\hline & \multicolumn{2}{c}{ Garnet in garnetite } & & \multicolumn{3}{c}{ Garnet in quartz } \\
\cline { 2 - 3 } \cline { 5 - 7 } & \multicolumn{1}{c}{ I } & II & & III & IV & V \\
\hline Spessartine & 44.06 & 46.52 & & 46.21 & 50.25 & 58.27 \\
Almandine & 32.97 & 30.98 & & 29.65 & 27.50 & 19.90 \\
Grossular & 16.72 & 15.17 & & 19.00 & 15.63 & 16.80 \\
Pyrope & 5.98 & 6.95 & & 5.06 & 6.29 & 4.90 \\
Schorlomite & 0.27 & 0.37 & & 0.07 & 0.33 & 0.13 \\
\hline
\end{tabular}

silicification is retrograde-metamorphic and could have taken place under temperatures significantly higher than $300^{\circ} \mathrm{C}$. Similar to Zadní Hutisko (Fig. 15a), silicification of garnetite was also detected in Mnišské jámy and, in particular, in Kyzový důl, where coarse-grained and mostly corroded garnet relics have been preserved in late quartz.

In order to confirm the results of the petrographic investigations, which showed that the rocks contain more than only one quartz generation of which the later, widely represented within the rocks, is of low-temperature origin, cathodoluminescence microscopy was utilized.

The greatest amount of quartz, described as late quartz, occurs in all deposits including Rudná hora and Sylvani. In accordance with its low temperature of formation (about $350^{\circ} \mathrm{C}$ ), the trace-element concentration is low producing weak $\mathrm{CL}$ emissions. The luminescence is brownish violet, i. e. a combination of reddish and bluish CL emission caused by a variety of defect structures in the quartz lattice (van den Kerkhof 2003) (Figs 30a $/ \mathrm{a}_{2}$ and $\left.b_{1} / b_{2}\right)$. The $C L$ investigations of late quartz were also carried out along the contact to garnetite (Fig. $30 \mathrm{c}_{1} / \mathrm{c}_{2}$ ) as illustrated in Fig. 15a. The image in CL mode shows that (1) the quartz groundmass of garnetite is characterised by bright and slightly bluish or greenish colours probably due to its relatively high Ti, Fe and Mn contents (Müller 2000) and (2) this late quartz resembles late quartz of the other samples (compare Fig. $30 \mathrm{c}_{1} / \mathrm{c}_{2}$ with Figs $30 \mathrm{a}_{1} / \mathrm{a}_{2}$ and $\mathrm{b}_{1} / \mathrm{b}_{2}$ ). As a sign of its secondary nature, late quartz contains relics of quartz originally associated with the garnetite. The latter may be also concentrated around recrystallized garnet grains (Fig. 30 $\mathrm{c}_{2}$ ). The $\mathrm{CL}$ images of rocks from Kosaře (Fig. $30 \mathrm{~d}_{1} / \mathrm{d}_{2}$ ) show that the quartz is represented by two generations: (1) bright red with patches of blue, and (2) relatively dark brownish-violet luminescing grains. The bright red luminescence is probably caused by high $\mathrm{Fe}^{3+}$ contents. In magmatic quartz, the average $\mathrm{Fe}$ concentration normally increases with the quartz formation temperature (Müller 2000). The first generation, not observed in the other deposits, is of high temperature origin - probably magmatic. The second generation is late quartz similar to that illustrated in the Figs $30 \mathrm{a}_{2}, \mathrm{~b}_{2}$ and $\mathrm{c}_{2}$ typical for low-grade metamorphic quartz.

\subsection{Comparison with Lahn-Dill deposits}

Pouba (1960 and 1970) inferred that the iron mineralizations of this study are identical with iron-formations. Fojt (2003) was of the same opinion, stating that the iron mineralizations are meta-exhalites, genetically related to the Lahn-Dill type. As an example of the well-known Lahn-Dill type deposits of the same area, we selected the Malý Děd Mt. occurrence, which belongs to the upper of the nappes overlying the Desná Dome. The rocks, which are of Devonian age, consist of quartzites, mica schists and metavolcanic rocks, and are an equivalent of the Vrbno Group (Cháb et a. 1984; Schulmann and Gayer 2000). For comparison, we have selected and analysed four minerals from the rocks of Malý Děd:

- amphiboles are $\mathrm{Fe}-\mathrm{Mg}$ amphiboles, corresponding to grunerite (Tab. 3, XIII and XIV), with or without tschermakite relics (Tab. 3, XII) in the centre of the grunerite grains. As a sign of their exhalative origin, their $\mathrm{Mg}$ numbers are below 0.2, i.e. lower than the limit of 0.4 defined by Mücke (2003) (Fig. 31);

- chlorite is iron-rich chamosite (Tab. 5, XV and XVI) and have compositions comparable with chlorites from iron-formations (Fig. 28);

- carbonate of the dolomite group is Fe-rich, containing on average 84.6 mol. \% ankerite (Tab. 10, last row);

- apatite is pure hydroxyl-apatite.

The investigation of the Malý Děd Mt. amphibole, chlorite, carbonate and apatite show compositions unlike at any of the localities in this study, being instead typical for metamorphically overprinted submarine-exhalative deposits of the Lahn-Dill type. This is considered in the American literature to belong to the youngest members of Algoma-type iron-formations (e.g. Hutchinson 1992).

\subsection{Genetic considerations}

Many observations and data obtained in course of this study (e.g. the chemistry of amphibole, chlorite, apatite and enriched magnetite) shows that the iron mineralizations of the Desná Group differ from typical iron-formations. However, whole-rock and mineral compositions reveal that they resemble those occurring within the rocks of the Sobotín Massif (including Sylvani and Rudná hora). These similarities suggest that they all originated most probably from the same source - the Sobotín Massif. As a consequence, the iron mineralizations within the gneisses have a striking symmetric arrangement around the main body of the Sobotín Massif (Fig. 1), a pattern which was also noticed by Pouba (1960). 

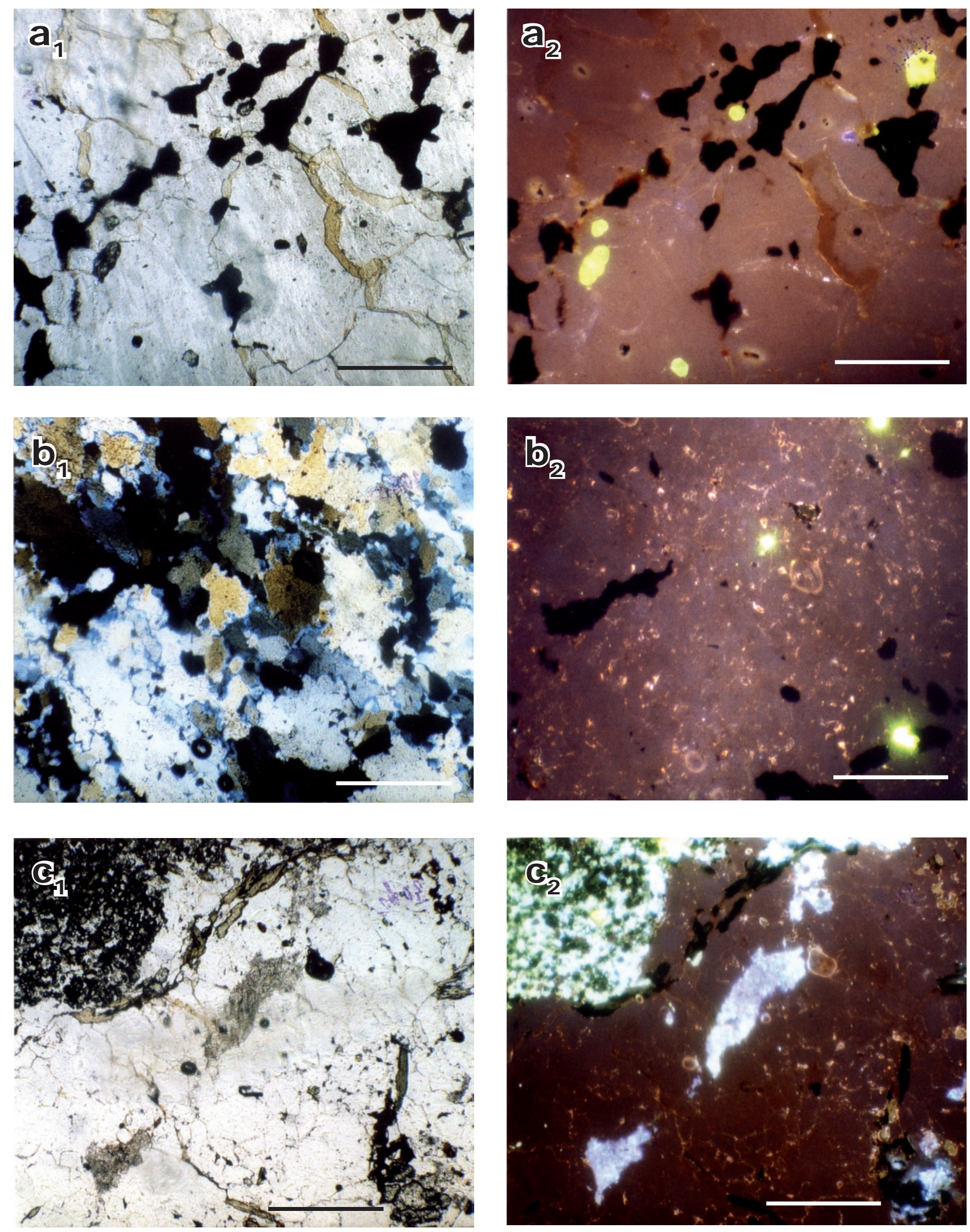

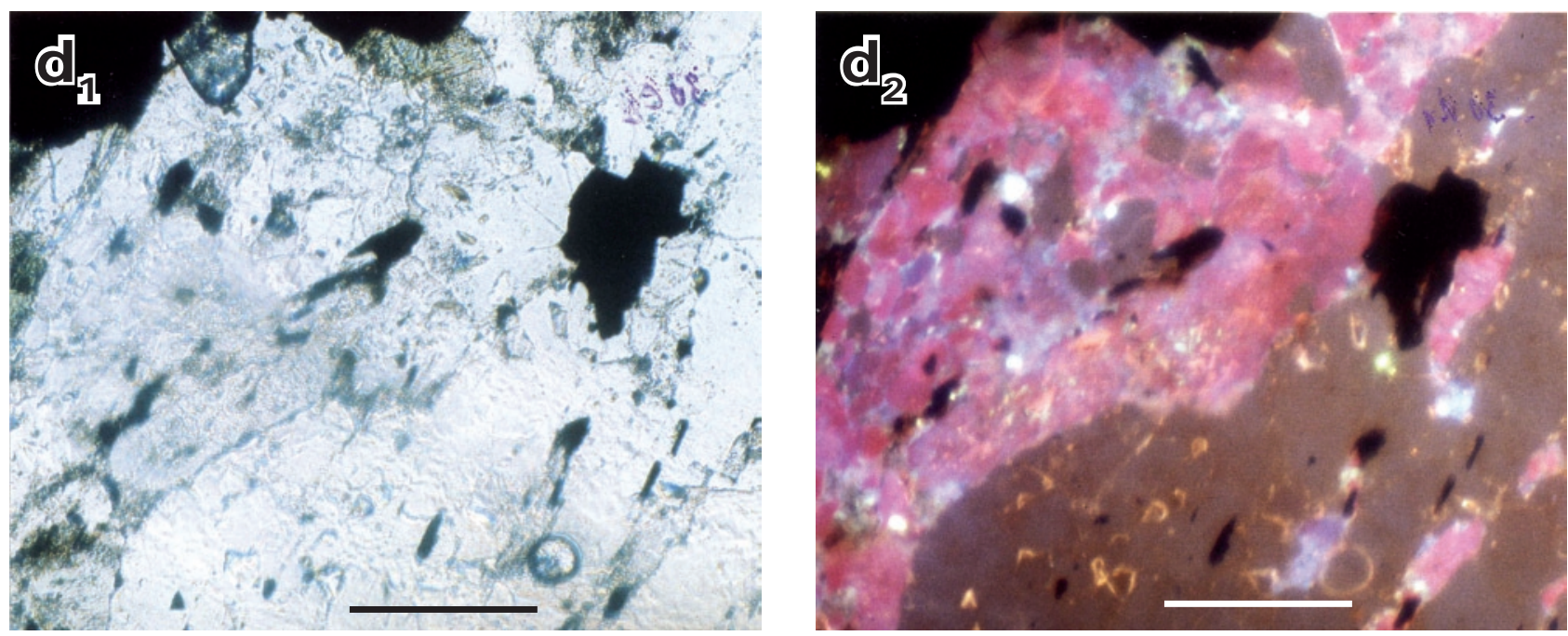

Fig. 30 Optical (transmitted light, index 1) and corresponding cathodoluminescence (CL, index 2) photomicrographs of the studied ores. $\mathbf{a}_{1}$ - Quartz containing opaque magnetite inclusions. Plain polarized light. Scale bar $0.5 \mathrm{~mm}$; Rudná hora.

$\mathbf{a}_{2}$ - Late quartz (dark brownish violet CL) enclosing magnetite (black) and apatite (bright and yellow). Scale bar 0.5 mm; Rudná hora.

$\mathbf{b}_{\mathbf{1}}-$ Quartz with inclusions of magnetite (opaque). Crossed polars, scale bar $0.5 \mathrm{~mm}$; Mnišské jámy.

$\mathbf{b}_{2}$ - Late quartz (dark brownish violetluminescing) containing magnetite (black) and apatite (bright and yellow) inclusions. Scale bar 0.5 mm; Mnišské jámy.

$\mathbf{c}_{1}-$ Contact between garnetite (upper part) and quartz, which contains chlorite (dark brown and elongated) with garnet (right side, lower part). Plain polarized light. Scale bar $0.5 \mathrm{~mm}$; Zadní Hutisko.

$\mathbf{c}_{2}$ - Late quartz (dark brownish violet CL) in contact to garnetite with a quartz (bright with bluish colour) groundmass containing garnet crystals. Note the relics of garnetite-quartz in late quartz generation and around recrystallized garnet. Scale bar $0.5 \mathrm{~mm}$; Zadní Hutisko.

$\mathbf{d}_{1}$ - Compact magnetite (opaque, upper part) and quartz enclosing magnetite grains. Plain polarized light. Scale bar $0.25 \mathrm{~mm}$; Kosaře.

$\mathbf{d}_{2}$ - Cathodoluminiscence image showing two quartz-generations: (1) high-temperature quartz, relatively bright red luminescing, in contact to compact magnetite, and (2) late, low-temperature, dark brownish violet quartz. Scale bar $0.25 \mathrm{~mm}$; Kosaře.

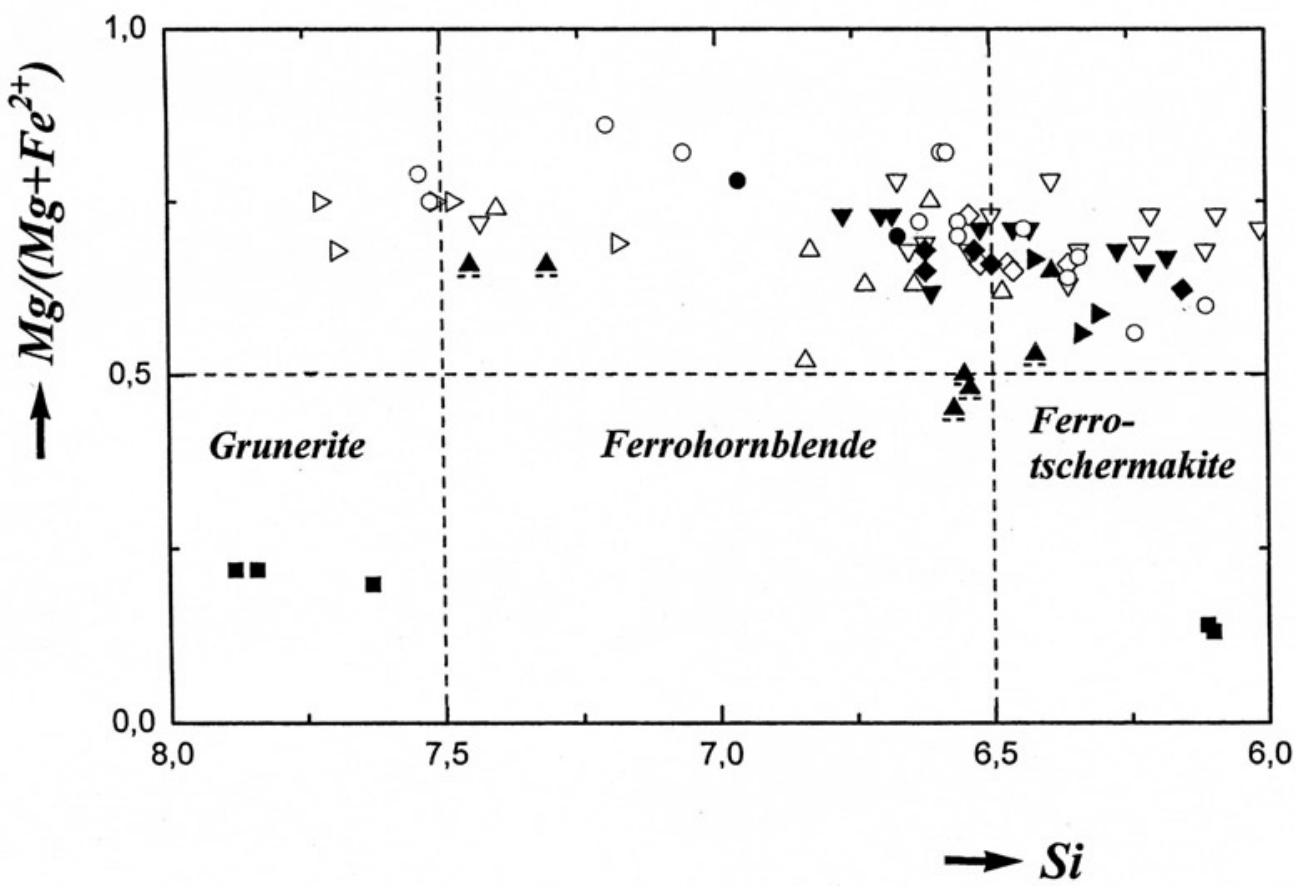

Fig. 31 Amphibole classification diagram $\mathrm{Mg} /(\mathrm{Mg}+\mathrm{Fe})$ vs. Si (apfu) (compare Fig. 16) of Leake et al. (1997). It is designed for amphiboles with $\mathrm{Ca}_{\mathrm{B}} \geq 1.50$ and $(\mathrm{Na}+\mathrm{K})_{\mathrm{A}}<0.50$, except for grunerite (Fe/Mg-amphibole), which contains $\mathrm{Ca}$ and $\mathrm{Na}$ as trace-elements only. In the ferro-tschermakite and grunerite fields plot analyses contain analytical points obtained from the Malý Děd occurrence (filled squares). 
It is inferred that the emplacement of the basic rocks of the Sobotín Massif was accompanied by the formation of apophyses, preferentially utilizing the schistosity and foliation of the gneisses. These apophyses contain, among other minerals (e.g., chlorite, biotite, epidote and albite), amphibole, magnetite and minor, but locally significant quantities of apatite (up to $20 \mathrm{vol}$. \%). The occurrence of apophyses with variable composition results most probably from the melt differentiation. At the early stage, the melt was characterized by low oxygen fugacity, which suppressed the formation of magnetite. Iron was predominantly bound in the $\mathrm{Fe}^{2+}$-bearing silicates - amphiboles and biotite. Later, oxygen fugacity increased as did consequently the $\mathrm{Fe}^{3+}$ activity, causing a progressive extraction of iron by steady magnetite separation from the melt and its enrichment due to gravity segregation. The enrichment in magnetite was accompanied by an increase in the apatite content, which was also locally enriched by gravity segregation. It is inferred that the high phosphorus contents acted as a flux, permitting a late low-temperature mobility of melts. It was also observed that magnetite-amphibole rocks contain epidote. In the Sobotín Massif, Fiala et al. (1980) found more than 25 vol. \% magnetite in amphibole-dominated rocks that were enriched in epidote. The anomalous iron concentration in these rocks was explained by post-magmatic migration (Fiala et al. 1980).

The formation of apophyses occurred at various stages after the emplacement of the basic rocks of the Sobotín Massif. Therefore, the amphibole-rich apophyses, both magnetite-bearing and nearly free of this mineral (= metahornblendite), may intersect each other. The abundance of amphibole-bearing veins in the vicinity of the Sobotín Massif is well-known and was recently mapped in a detail (as summarized in Fig. 32). This figure shows not only the abundance, but also the distribution of the apophyses, which are generally parallel both to each other and to the mapped magnetite-rich bands, indicating that the two rocks (hornblendite and magnetite-amphibole rocks) are of the same origin (compare Fig. 1 with Fig. 32 in the vicinity of Švagrov).

The garnetites are older than the magnetite-rich rocks and of the same age as the gneisses. Most probably, their protolith was formed from $\mathrm{Mn}$ - and Fe-rich submarine volcanogenic exhalations. Volcanogenic exhalations are well-known for many types of deposits (sulphides and oxides) and they may occur from the early Archaean to the present. In the American literature, oxidic iron deposits of exhalation origin are known as iron-formations that belong to the Algoma type (Gross 1980). In Europe, on the other hand, younger than Precambrian exhalative iron deposits are separated from the older Algoma type iron-formations and are designated as the Lahn-Dill type deposits, to which belongs the Malý Dĕd Mt. occurrence.
As the continued use of the names Algoma and Lake Superior type of Gross (1980) is not recommended, the new classification of Trendall (2002) will be followed. Trendall (2002) distinguished between banded ironformation (BIF) and granular iron-formation (GIF). In terms of this classification, the Algoma type is more or less identical to the GIF.

In the area under study, the exhalations occurred contemporaneously with the sedimentation of the protolith to the gneisses, corresponding to greywackes (Fojt 2003) considered to be Proterozoic (Pouba 1970). This age was recently confirmed by Kröner et al. (2000): the Desná gneisses, derived from the metagraywacke protolith, contain detrital zircons with $\mathrm{Pb}-\mathrm{Pb}$ ages scattering between $628.8 \pm 1.5$ and $664.8 \pm 0.8 \mathrm{Ma}$. In contact with sea water, the volcanogenic exhalations became unstable, and the $\mathrm{Mn}-\mathrm{Fe}-$ rich, mudstone-like precipitates formed. These were deposited as mixtures with the above-mentioned continental-derived detrital greywacke material. The phases free of volcanic activity are characterized by sediments of pure greywackes. Due to subsequent polyphase pre-Variscan metamorphism reaching up to amphibolite facies conditions (Fediuková et al. 1988), the greywackes were transformed into paragneisses and the mixtures into garnetiferous rocks, which are granular iron-formations equivalent to coticules.

The term coticules was coined by Renard (1878), who defined the rock as essentially containing spessartine-almandine garnet in a groundmass of sericite and/or quartz. Coticules are typically, but not necessarily, associated with rift-hosted base metal sulphide deposits and they result either from exhalative processes or sea-floor replacement (Spry et al. 1997). Other minerals known from coticules are, among others, muscovite, biotite, chlorite, feldspar, ilmenite and alumosilicates. It was suggested by many authors (Boström 1973; Peter and Goodfellow 1996; Spry et al. 1997) that the protoliths of coticules were derived predominantly from hydrothermal sources with detrital material admixture.

In Fig. 25, the garnetites plot exactly in the field of coticules defined by Spry et al. (1997). These authors stated that the chondrite-normalized REE patterns for coticules are similar to those for iron-formations. The metamorphosed rocks are generally enriched in light and depleted in heavy REE. Coticules, like iron-formations, may also display negative $\mathrm{Ce}$ anomalies as well as positive or negative Eu anomalies. These data are in line with our results, summarized in Fig. 27.

During the second tectonometamorphic event, all the discussed rock types were overprinted by Variscan Barrovian metamorphism ranging from the chlorite zone in the east (identical to the studied part of the Desná Group; $300-350^{\circ} \mathrm{C}$ at $2-3 \mathrm{kbar}$ ) to the staurolite zone in the NW part of the Keprník Unit (Fediuková et al. 1988). 


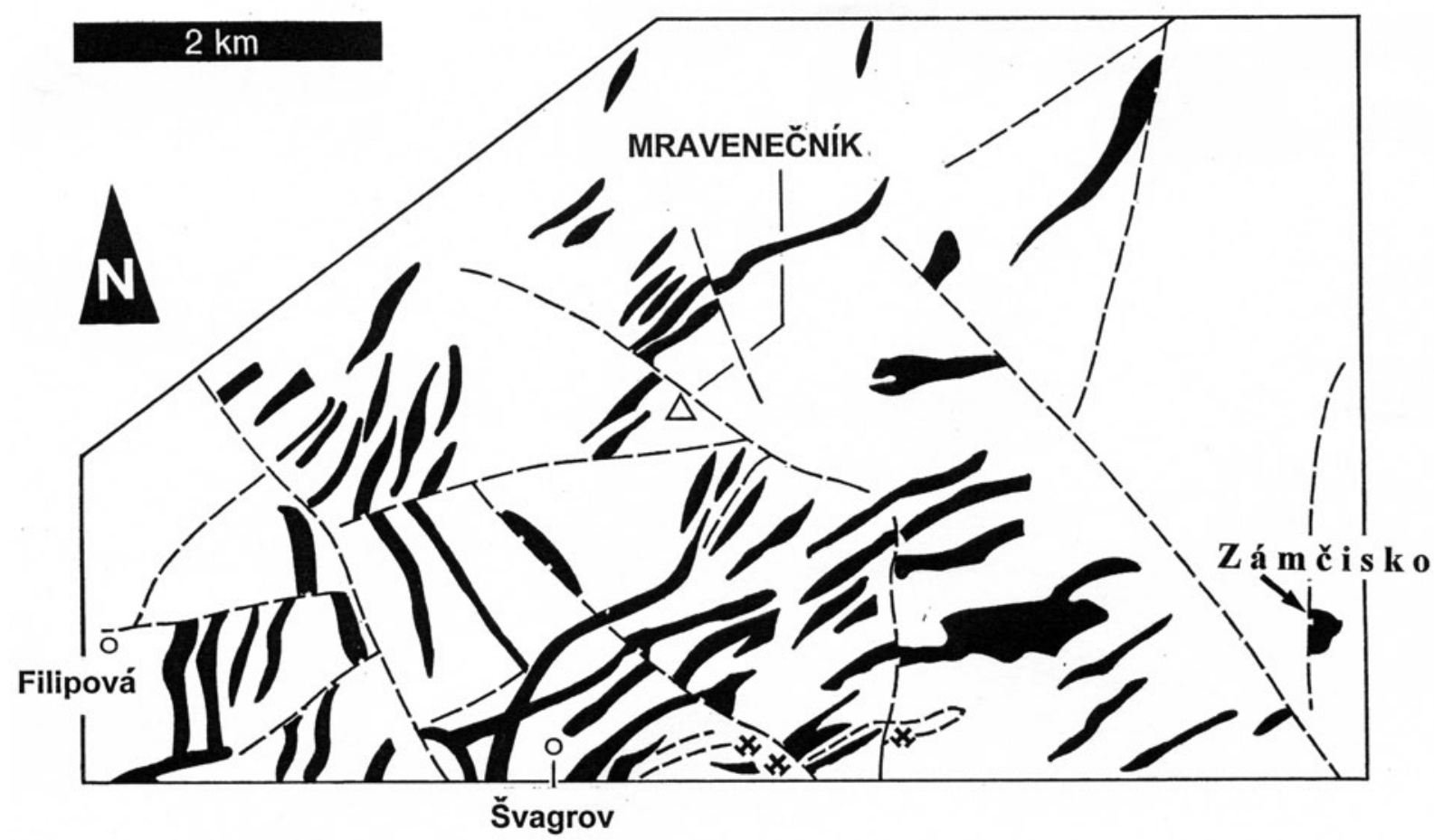

Fig. 32 Distribution of apophyses and dykes within the Desná gneisses, re-drawn from the Geological Map of CR, sheet 14-24 (Bělá pod Pradědem), published by the Czech Geological Survey in 1997.

In the Desná gneisses, chloritization of biotite was the most prominent, whereas magnetite recrystallized just to some extent and the associated minerals (amphibole and biotite) were slightly aligned and/or foliated and replaced by newly formed minerals (chlorite, actinolite, albite, carbonate and epidote). Under retrograde conditions of metamorphism, the rocks were silicified to a various degree. Due to the fact that silicification affected amphibole rather than magnetite, the original proportion of rock-forming minerals changed - the amount of quartz increased predominantly at the expense of amphibole. Rocks composed solely of quartz and magnetite do not occur. Relics of amphibole (or biotite, chlorite) with corroded and irregular grain boundaries are always preserved in the invading late quartz.

\section{Conclusions}

By definition, iron-formations are of sedimentary origin. However, the magnetite-rich rocks of the Desná Group in the Silesicum seem to have formed under magmatic conditions. They are interpreted as metamorphosed and silicified equivalents of iron-rich basic rocks and, thus, similar to the Itakpe-type deposit of Nigeria (Mücke and Neumann 1986), which was also formerly described as iron-formation (Olade 1978).
Acknowledgements Electron-microprobe analyses were carried out at the Institut für Mineralogie und Mineralische Rohstoffe of the University of Clausthal by K. Hermann. We are grateful to him. We are also indebted to G. Hartmann and K. Simon (both of the Geochemisches Institut) and to W. van den Kerkhof (Geologisches Institut) of the Georg-August University of Göttingen, for XRF and ICP-MS analyses and cathodoluminescence microscopy. We would also like to thank H. Büttner for transforming the data into diagrams. In particular, we are grateful to B. Fojt, Masaryk University, Brno, for his well-organized excursions to the small and hidden deposits, for his introduction into the geology and mineralogy of the area, the various discussions about these complex deposits and for providing some characteristic samples.

One of the authors (Z. Losos) is highly indebted to the Konferenz der Deutschen Akademien der Wissenschaften, which offered him a 7 months scholarship in Göttingen University, sponsored by the Volkswagen-Stiftung. The project was supported partly also by the grant 663 MSM0021622412 of the Czech Ministry of Education. We are grateful to Z. Pouba, Prague, and the Moravian Museum, Brno, for providing some rock samples. In addition we also acknowledge the critical reviews of the manuscript by Sharon Webb (Georg-August-University), an unknown reviewer and in particular by V. Janoušek and S. Vrána, whose critical comments led to a substantial improvement of the paper. 


\section{References}

Alibert C, McCulloch MT (1993) Rare earth element and neodymium isotopic composition of the banded iron-formations and associated shales from Hamersley, Western Australia. Geochim Cosmochim Acta 57: 187-204

BaAs Becking IGM, Kaplan I R, Moore D (1960) Limits of natural environment in terms of $\mathrm{pH}$ and oxidationreduction potentials. J Geol 68: 243-284

BEDERKE E (1938) Die Eisenerzlagerstätten der östlichen Sudeten. Z. Berg-, Hütten- und Salinenwesens 86: 481-487

Beukes NJ, Gutzmer J (1996) A volcanic-exhalative origin for the world's largest (Kalahari) manganese field. Miner Depos 31: 242-245

BostRÖM K (1973) Submarine volcanism as a source for iron. Earth Planet Sci Lett 9: 348-354

BÜHN B, StANISTREET IG (1997) Insight into the enigma of Neoproterozoic manganese formations from the perspective of supercontinental break-up and glaciation. In: Nicholson K, Hein K, Bühn B, Dasgupta S (eds) Manganese mineralizations: geochemistry and mineralogy of terrestrial and marine deposits. Geol Soc London Spec Pub 119: pp 81-90

Cháb J, Fišera M, Fediuková E, Novotný P, Opletal M, SKÁCELOVÁ D (1984) Problems of the tectonic and metamorphic evolution of the eastern part of the Hrubý Jeseník Mts. (Altvatergebirge), northern Moravia, Czechoslovakia. Sbor geol Věd, Geol 39: 27-72 (in Czech)

Edfelt A, Armstrong RN, Smith M, Martinsson O (2005) Alteration paragenesis and mineral chemistry of the Tjårrojåkka apatite-iron and $\mathrm{Cu}(-\mathrm{Au})$ occurrences, Kiruna area, northern Sweden. Miner Depos 40: 409-434

Fediuková E, FišERA M, ChÁB J, KopeČNÝ V, RyBKa R (1988) Garnets of the Predevonian rocks in the eastern part of the Hrubý Jeseník Mts. North Moravia, (Czechoslovakia). Acta Univ Carol, Geol 3: 197-234

Fiala J, Jelínek E, Pouba Z, Poubová M, Souček J (1980) The geochemistry of the ultrabasic rocks of the Sobotín amphibolite Massif (Czechoslovakia). Neu Jb Mineral, Abh 137: 257-281

FoJT B (2002) Banded iron ores in the Desná gneisses of Silesicum: Review of genetic interpretations, a present state of knowledge. In: Mineralogie Českého masívu a Západních Karpat, Olomouc, Abstract Volume, pp 18-25 (in Czech)

Fojt B (2003) Genetische Probleme der gebänderten Eisenerzlagerstätten: Ein Beispiel aus der Tschechischen Republik. Mitt Österr mineral Gesell 148: 143-145

Fojt B, Hladíková J, Kopa D, Krausová D, Sulovský P, VÁVRA V, ZıмÁK J (1997) Mineral and rock association of the Zámčisko quarry, Hrubý Jeseník Mountains, Czech Republic. Čas. Slez. Muz. Opava (A) 46: 235-264 (in Czech)
Fojt B, Kopa D, Sulovský P, ŠKoda R, VÁvra V (2004) The mineral association at the contact between the amphibolites and banded iron ores in Desná Gneisses (Hrubý Jeseník Mountains). In: Mineralógia Západných Karpát a Českého Masívu, Abstract Volume, Bratislava, pp 30-31 (in Czech)

Glasby GP (1997) Fractionation of manganese from iron in Archaean and Proterozoic sedimentary ores. In: Nicholson K, Hein K, Bühn B, Dasgupta S (eds) Manganese Mineralizations: Geochemistry and Mineralogy of Terrestrial and Marine Deposits. Geol Soc London Spec Pub 119: pp 29-42

Gross GA (1980) A classification of iron formations based on depositional environments. Canad Mineral 18, 215-222

Haggerty SE (1976) Opaque mineral oxides in terrestrial igneous rocks. In: El Goresey A, Haggerty SE, Huebner JS, Lindsley DH, Rumble D (eds) Oxide Minerals, Min Soc Am, Short Course Notes, Vol 3, p Hg 153

Heinrichs H, Herrmann AG (1990) Praktikum der Analytischen Geochemie. Springer Verlag Berlin Heidelberg, pp $1-420$

HutChinson RW (1992) Mineral deposits and metallogeny: indicators of Earth's evolution. In: Schidlowski M (ed) Early Organic Evolution: Implications for Mineral and Energy Resources. Springer Verlag, Berlin, pp 521-545

Klemm DD (2000) The formation of Palaeoproterozoic banded iron formations and their associated Fe and Mn deposits, with reference to the Griqualand West deposits, South Africa. J Afr Earth Sci 30: 1-24

KoRZHINSKY MA(1982) Apatite solid solutions as indicators of the fugacity of $\mathrm{HCl}$ and $\mathrm{HF}$ in hydrothermal fluids. Geochem Int 3: 45-60

KRAUSKOPF KB (1957) Separation of manganese from iron in sedimentary processes. Geochim Cosmochim Acta 12: $61-84$

Kretschmer R (1911) Die metamorphen Diorit- und Gabbromassive in der Umgebung von Zöptau (Mähren). Jb Geol Reichsamt 61: 53-179

Kröner A, ŠTíPskÁ P, Schulmann K, JAeCKel P (2000) Variscan evolution of the northeastern margin of the Bohemian Massif, Czech Republic. In: Franke W, Haak V, Oncken O, Tanner D (eds) Orogenic Processes: Quantification and Modelling in the Variscan Fold Belt. Geol Soc London Spec Pub 179: pp 175-197

Leake BE, Woolley AR, Arps CES, Birch WD, Gilbert MC, Grice JD, Hawthorne C, Kato A, Kisch HJ, KrivovicheV VG, Linthout K, Liard J, Mandarion J, Maresch WV, Nickel EH, Rock NMS, Schumacher JC, Smith DC, Ungaretti L, Whittacker EJW, Youzhi G (1997) Nomenclature of amphiboles: Report of the subcommittee on Amphiboles of the International Mineralogical Association Commission on New Minerals and Mineral Names. Eu J Mineral 9: 623-642 
LIARD J (1988) Chlorites: metamorphic petrology. In: Bailey SW (ed) Hydrous Phyllosilicates, Review in Mineralogy 19: 405-447

LOBERg BEH, HoRndahl AK (1983) Ferride geochemistry of Swedish Precambrian iron ores. Miner Depos 18: 487-504

MatTHES S (1961) Ergebnisse zur Granatsynthese und ihre Beziehungen zur natürlichen Granatbildung innerhalb der Pyralspit-Gruppe. Geochim Cosmochim Acta 23: 233-294

MeLKa K (1965) Proposal of chlorite classification. Věst Ústř úst geol 40: 23-29

MÜCKE A (2003) General and comparative considerations of whole-rock and mineral compositions of Precambrian iron-formations and their implications. Neu Jb Mineral, Abh 179: 175-219

MüCKE A (2006) Chamosite, siderite and the environmental conditions of their formation in chamosite-type Phanerozoic ooidal ironstones. Ore Geol Rev 28: 235-49

MÜCKE A, FARSHAD F (2005) Whole-rock and mineralogical composition of Phanerozoic ooidal ironstones. Comparison and differentiation of types and subtypes. Ore Geol Rev 26: 227-262

MǘKE A, Losos Z (1999) The significance of chlorite as a genetic indicator in ore deposits exemplified by the magnetite ores from the Desná Group (Silesicum, Czech Republic). Chem Erde 59: 233-242

MǗKE A, Losos Z (2000) Polymetamorphic overprinting and silicified banded magnetite ores from the Desná gneisses (Silesicum, Czech Republic). Acta Mus Moraviae, Sci Geol LXXXV: 47-80 (in Czech)

Mücke A, Losos Z (2002) The genesis of the Precambrian so-called iron formations (Sydvaranger type) within the gneiss of the Desná Dome, Hrubý Jeseník Mountains, Czech Republic. Mineralogical Society of Poland, Special Papers 20: 153-155

Mücke A, Neumann U (1986) The genesis of the banded iron ores deposit of Itapkpe area, Kwara State, Nigeria. Fortschr Mineral 64: 187-204

MülLER A (2000) Cathodoluminescence and characterization of defect structures in quartz with application to the study of granitic rocks. Unpublished $\mathrm{PhD}$ thesis, Univ Göttingen: pp 1-222

Neuser RD, Bruhn F, Götz J, Habermann D, Richter KD (1995) Kathodolumineszenz: Methodik und Anwendung. Zbl Geol Paläont Teil I H 1/2: 287-306

Olade MA (1978) General features of a Precambrian iron ore deposit and its environment at Itakpe ridge, Okene, Nigeria. Inst Miner Metallurgy, Trans Sec B 87: 1-9

Peter JM, Goodfellow WD (1996) Mineralogy, bulk and rare earth element geochemistry of massive sulphideassociated hydrothermal sediments of the Brunswick horizon, Bathurst mining camp, New Brunswick. Can J Earth Sci 33: 252-283
Pouba Z (1960) Magnetite ores of Sydvaranger type in Czechoslovakia. Int Geol Cong XXI session 16: pp 164-170

Pouba Z (1970) Pre-Cambrian banded magnetite ores of the Desná Dome. Sbor Geol Věd, ložisk Geol Mineral 12: 7-64

Pouba Z, Zoubek V (1985) Precambrian metallogeny of Central Europe. In: International Conference on Metallogenesis of the Precambrian in Europe, Prague: pp 7-55

Pouchou JL, Pichoir F (1984) A new model for quantitative X-ray microanalysis. Part I: Application to the analysis of homogenous samples. Rechérches Aérospatiale 3: 13-38

RAмдонr P (1975) Die Erzmineralien und ihre Verwachsungen, Akad-Verlag Berlin, pp 1-1036

Renard A (1878) Sur la structure et la composition minéralogique du coticules et sur ses rapports avec le phyllade oligistifère. Mémoires couronnés de l'Académie Royale Belgique 41: pp 1-42

Rieder M, Cavazzini G, D, Yakonov Y, Gottardi G, GugGenheim S, Koval PV, Müller G, Neiva AMR, Radoslovich EW, Robert JL, Sassi FP, Takeda H, Weiss Z, Wones DR (1998) Nomenclature of the micas. Canad Mineral 36: 905-912

SelLner Z (1930) Die Magnetitlagerstätten der tschechoslowakischen Republik. III: Die Vorkommen im Altvater, a) Mönchschächte bei Winkelsdorf. Ztschr Prakt Geol 38/7: 97-104

Schulmann K, GaYer R (2000) A model for a continental accretionary wedge by oblique collision. J Geol Soc, London 157: 401-416

SCHUMACHeR JC (1997) The estimation of ferric iron in electron microprobe analysis of amphiboles. Eu J Mineral 9: 643-651

Spry PG, Peter J M, Slack JF (1997) Meta-exhalites as exploration guides to ore. Geol Survey of Canada, Contribution no. 1997162: pp 163-201

Taylor SR, McLennan SM (1985) The Continental Crust: Its Composition and Evolution. Blackwell, Oxford, pp $1-312$

TRENDAll AF (2002) The significance of iron-formation in the Precambrian stratigraphic record. In: Altermann W, Corcoran PL (eds) Precambrian Sedimentary Environments: a Modern Approach to Depositional Systems. Blackwell, Oxford, I. A. S., Spec Pub 434: 33-66

VAN DEN KeRKHOF AM (2003) Advancements of cathodoluminescence microscopy and related techniques with applications to the study of fluid-rock interaction. Festschrift 70. Geburtstag Prof. Dr. Hans-Jürgen Behr, Göttinger Arb Geol Paläont Sb 5: 111-119

WERNER CD (1984) Global evolution of the mafic magmatism with special regard to the rare earth elements. Miner Slov 16: 29-37 
ZimÁK J, KROPÁČ K (2006) Mineralogy of metamanganolites in BIF of the Desná Group. Mineral Polonica, Special Papers 28: 245-247

ŽÁK L (1974) Comparison of Proterozoic Mn horizons in the Jeseníky Mts. and Železné hory Mts. In: Sborník
Korelace proterozoických a paleozoických stratiformních ložisek II: pp 24-35 (in Czech)

ŽÁK L (1975) Mineral assemblage of Proterozoic Mn horizons in the Jeseníky Mts. and Železné hory Mts. In: Sborník Korelace proterozoických a paleozoických stratiformních ložisek III: pp 17-24 (in Czech) 